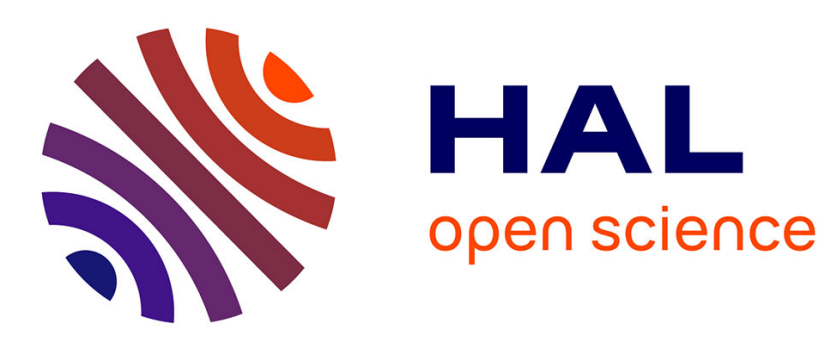

\title{
Arbitrage transnational et droit international général (2016)
}

Patrick Jacob, Franck Latty, Arnaud de Nanteuil

\section{To cite this version:}

Patrick Jacob, Franck Latty, Arnaud de Nanteuil. Arbitrage transnational et droit international général (2016). Annuaire Français de Droit International, 2017, pp.587-650. hal-01783005

\section{HAL Id: hal-01783005 \\ https://hal.parisnanterre.fr/hal-01783005}

Submitted on 8 May 2018

HAL is a multi-disciplinary open access archive for the deposit and dissemination of scientific research documents, whether they are published or not. The documents may come from teaching and research institutions in France or abroad, or from public or private research centers.
L'archive ouverte pluridisciplinaire HAL, est destinée au dépôt et à la diffusion de documents scientifiques de niveau recherche, publiés ou non, émanant des établissements d'enseignement et de recherche français ou étrangers, des laboratoires publics ou privés. 


\title{
ARBITRAGE TRANSNATIONAL ET DROIT INTERNATIONAL GÉNÉRAL (2016)
}

\author{
PATRICK JACOB* ${ }^{*}$ FRANCK LATTY** \\ et ARNAUD DE NANTEUIL****
}

Il ne sera pas dit que l'année 2016, pourtant traversée par de multiples débats quant à la " légitimité » de l'arbitrage transnational, aura marqué un quelconque déclin de celui-ci. Au cours de l'année, 62 nouvelles instances ont été engagées et 57 décisions ont été rendues sur le fond, dont 41 ont été rendues publiques ${ }^{1}$. Ces chiffres traduisent des tendances stables, même si le nombre d'instances introduites est sensiblement plus élevé que celui des années précédentes ${ }^{2}$. Ils font en tout cas état d'un certain dynamisme, lequel s'est également manifesté par la signature de l'Accord économique et commercial global (CETA suivant son acronyme anglais) entre l'Union européenne et le Canada le 30 octobre $2016^{3}$. Le processus de ratification n'a pas encore débuté, mais l'on sait d'ores et déjà que sa nature « mixte " au regard du droit de l'Union européenne devrait impliquer une ratification non seulement de l'Union elle-même mais également de chacun des États membres, conformément à leurs dispositions constitutionnelles ${ }^{4}$. Son avenir n'est donc pas pleinement garanti, mais le fait qu'il ait été cité à plusieurs reprises par la jurisprudence arbitrale en 2016, à titre d'illustration, montre que cet accord important est en train de prendre sa place dans le corpus normatif applicable à l'investissement international ${ }^{5}$. C'est également cette année qu'a été signé le partenariat transpacifique, signe que la multilatéralisation du droit des investissements pourrait se confirmer, en dépit du retrait des États-Unis d'Amérique de cet accord sitôt leur nouveau président entré en fonctions. De manière plus ponctuelle, mais ce n'est pas sans importance, c'est en 2016 qu'une réponse a été apportée à certaines des critiques les plus virulentes adressées à l'arbitrage d'investissement, notamment à travers l'affaire Philip Morris c. Uruguay, dans laquelle le tribunal a fermement rejeté la demande de l'investisseur dirigée contre les mesures sanitaires adoptées

\footnotetext{
(*) Professeur à l’Université de Versailles Saint-Quentin-en-Yvelines.

(**) Professeur à l’Université Paris Nanterre, directeur du Centre de droit international de Nanterre (CEDIN)

$\left(^{* * *}\right)$ Professeur à l'Université Paris-Est Créteil Val-de-Marne (Paris 12).

1. CNUCED, Investor-State Dispute Settlement : Review of Developments in 2016, IIA Issue note, 2017-1, p. 2. Il faut en outre préciser qu'un certain nombre de décisions rendues à la fin de l'année 2015 n'ont été rendues publiques qu'en 2016 et sont, pour cette raison, intégrées à cette chronique.

2. Ibid. Il est relevé qu'en moyenne, sur ces dix dernières années, le nombre de nouvelles instances introduites s'élève à 49 .

3. Le texte français du traité est disponible sur le site de la Commission européenne: http://ec.europa. eu/trade/policy/in-focus/ceta/index_fr.htm.

4. C'est la conclusion à laquelle est parvenue la Cour de justice de l'Union européenne dans son avis 2/15 rendu le 16 mai 2017 au sujet de l'accord de libre-échange entre l'UE et Singapour. Il paraît donc difficile de contester que cette solution doit être applicable au CETA lui-même, ce que la Commission européenne avait admis sur le plan politique.

5. CIRDI, Philip Morris Brands SARL, Philip Morris Products SA c. Uruguay, $\mathrm{n}^{\circ} \mathrm{ARB} / 10 / 7$, sentence, 8 juillet 2016, § 300 ; CIRDI, Saint-Gobain Performance Plastics Europe c. Venezuela, n ARB/12/13, décision sur la responsabilité et les principes de la réparation, 30 décembre $2016, \S 489$.
} 
par le gouvernement défendeur ${ }^{6}$. Il n'est pas impossible que l'ensemble de ces éléments révèlent une évolution structurelle du droit international des investissements, à la fois par une meilleure structuration normative via le dépassement des traités bilatéraux et par une meilleure prise en compte des intérêts de l'État, qui était toutefois déjà présente dans la jurisprudence passée mais se trouve définitivement affirmée ${ }^{7}$.

Comme les années précédentes, les décisions de 2016 ont été au demeurant l'occasion pour les tribunaux arbitraux de faire application de nombreuses normes et concept du droit international public et de contribuer efficacement au dynamisme de celui-ci. L'importance sans cesse grandissante des questions procédurales, en particulier, ne doit pas occulter certains développements particulièrement stimulants en matière de contentieux international, lesquels peuvent eux-mêmes soulever des enjeux théoriques de grande envergure à l'instar de la question de la personnalité juridique internationale des investisseurs (III). Mais la question des sources (I) et de la responsabilité (II) sont également de nature à intéresser les internationalistes, bien au-delà du seul cercle des spécialistes du droit de l'investissement ${ }^{8}$.

\section{I. - ARBITRAGE TRANSNATIONAL ET SOURCES DU DROIT INTERNATIONAL}

La nomenclature des modes de formation du droit international censée être reflétée par l'article 38 du Statut de la Cour internationale de Justice est mal en phase avec les sources du « droit vivant » mis en œuvre par les tribunaux d'investissement. Certes, les traités constituent l'origine première des normes appliquées (A) tandis que la coutume (B) reste fort présente, aidée par un certain laxisme méthodologique des arbitres lorsqu'ils s'emploient à en déterminer le contenu. S'épanouissent par ailleurs de nombreux principes généraux dont l'appartenance à la catégorie de ceux « reconnus par les nations civilisées » peut prêter à discussion $(\mathrm{C})$, quand les normes issues de sources informelles, au sein desquelles on intégrera la doctrine, prospèrent $(\mathrm{D})$. En dépit de l'absence de règle du précédent ${ }^{9}$ (qu'une obsession du précédent largement partagée ${ }^{10}$ compense en dépit de limites tangibles ${ }^{11}$ ),

6. Ibid. Pour l'analyse de cet aspect de la sentence, voir notamment infra, II, A, $5^{\circ}$. Dans l'affaire Philip Morris c. Australie, la sentence rendue en décembre 2015 a conclu à une irrecevabilité et n'a donc pas abordé le fond. Pour une analyse, voir notamment infra, III, A, 3.

7. Voir cette chronique, cet Annuaire, 2010, pp. 607-654, pp. 609 et s.

8. La première partie a été rédigée par Franck Latty, la deuxième par Arnaud de Nanteuil et la troisième par Patrick Jacob.

9. CIRDI, Garanti Koza LLP c. Turkménistan, n ARB/11/20, sentence, 19 décembre 2016, § 149 ; Comité ad hoc CIRDI, TECO Guatemala Holdings LLC c. Guatemala, n ARB/10/23, décision sur l'annulation, 5 avril 2016, $\$ 237$.

10. CIRDI, Churchill Mining PLC et Planet Mining Pty Ltd c. Indonésie, $\mathrm{n}^{\circ} \mathrm{ARB} / 12 / 14$ et 12/40, sentence, 6 décembre 2016, $\S 236$ (bien que non lié par les précédents, le tribunal estime qu’il « must pay due consideration to earlier decisions of international tribunals"; que "subject to compelling grounds to the contrary, it has a duty to adopt principles established in a series of consistent cases "; et qu'il est de son devoir " to contribute to the harmonious development of international investment law, with a view to meeting the legitimate expectations of the community of States and investors towards legal certainty and the rule of law»). Dans le même sens, voir CIRDI, Vestey Group Limited c. Venezuela, $\mathrm{n}^{\circ} \mathrm{ARB} / 06 / 4$, sentence, 15 avril 2016, § 113. Rejetant l'argument d'une partie au motif qu'il n'a "no basis in the case law ", voir CIRDI, United Utilities (Talinn) BV et Aktsiaselts Tallinna Vesi c. Estonie, n ARB/14/24, décision sur la demande de mesures provisoires, 12 mai $2016, \S 93$.

11. Voir l'affaire CIRDI, Urbaser SA et Consorcio de Aguas Bilbao Bizkaia, Bilbao Biskaia Ur Partzuergoa c. Argentine, ${ }^{\circ} \mathrm{ARB} / 07 / 26$, sentence, 8 décembre 2016, qui partage les mêmes faits que l'affaire Impregilo (ARB/07/17, sentence du 21 juin 2011). Dans ce dernier cas, l'Argentine a été condamnée à hauteur de 21 millions $\$$; de l'affaire Urbaser, les investisseurs sortent bredouilles. 
la jurisprudence occupe une place telle qu'elle irrigue la mise en œuvre par les arbitres des normes provenant des autres sources. C'est de manière transversale qu'elle sera donc ici abordée ${ }^{12}$.

Plusieurs éléments parmi les développements qui suivent tendront ainsi à indiquer que sous l'accumulation de décisions arbitrales - celles rendues en 2016 s'inscrivent dans une tendance globale - plus ou moins transgressives, la nomenclature et, par-delà, les canons de la théorie classique des sources du droit international subissent de petits coups de boutoir qui contribuent à en faire évoluer la physionomie.

\section{A. Traités}

Le contentieux transnational d'investissement repose dans une très large mesure sur les droits tirés par les investisseurs de traités internationaux de protection des investissements. Les tribunaux arbitraux sont à ce titre conduits à faire vivre le droit des traités. En 2016 comme depuis vingt-cinq ans que les recours fondés sur les conventions d'investissement prospèrent, ce sont avant tout des questions d'interprétation de dispositions conventionnelles qui ont occupé les arbitres (1). Plus ponctuellement d'autres questions ressortissant au droit des traités se sont posées (2).

\section{Interprétation}

Si par nature la fonction juridictionnelle est interprétative, la marge de manœuvre du juge est plus ou moins étendue selon les textes qui sont soumis à son appréciation. Or c'est un fait que la plupart des traités d'investissement comportent des normes de protection à contenu fortement ou relativement indéterminé ${ }^{13}$ défaut que les traités les plus récents s'emploient à corriger en réduisant d'autant la marge d'interprétation des arbitres ${ }^{14}$. Au stade préliminaire, la compétence du tribunal pourra également être tributaire du sens qu'il donnera à des termes tels qu' investissement » ${ }^{15}$ ou " siège " ${ }^{16}$ contenus dans le traité applicable ou dans la convention de Washington s'agissant de l'arbitrage CIRDI.

De manière générale, les tribunaux arbitraux s'appuient explicitement sur les règles d'interprétation codifiées par la convention de Vienne sur le droit des traités, dont le caractère coutumier est rappelé ${ }^{17}$, justifiant de la sorte son application à des traités conclus avant son entrée en vigueur (cas de la convention de Washington) ${ }^{18}$

12. La catégorie des actes unilatéraux étatiques n'ayant pas donné lieu à des développements notables, elle ne sera pas traitée dans cette livraison.

13. Voir Yves NOUVEL, «Classification cursive des règles de fond issues des traités bilatéraux d'investissement ", RGDIP, 2015/1, pp. 8 et s.

14. Voir par exemple l'art. 8.10 de l'Accord économique et commercial global (AECG/CETA) entre l'Union européenne et le Canada relatif au traitement juste et équitable.

15. Ex. : CPA (CNUDCI), Flemingo Dutyfree Shop Private Ltd c. Pologne, sentence, 12 août 2016, $\S \S 294$ et $\mathrm{s}$.

16. Ex. : CIRDI, Tenaris SA et Talta-Trading e Marketing Sociedade Unipessoal Lda. c. Venezuela, $\mathrm{n}^{\circ} \mathrm{ARB} / 12 / 23$, sentence, 12 novembre 2016, $\S \S 183$ et s. ; CIRDI, Tenaris SA et Talta-Trading e Marketing Sociedade Unipessoal Lda. c. Venezuela, n ARB/12/23, sentence, 29 janvier 2016, § 154.

17. Ex. : CIRDI, Spence International Investments, LLC et al. c. Costa Rica, $\mathrm{n}^{\circ} \mathrm{UNCT} / 13 / 2$, sentence intérimaire, 25 octobre 2016, § 206 ; CIRDI, Tenaris SA et Talta-Trading e Marketing Sociedade Unipessoal Lda. c. Venezuela, $\mathrm{n}^{\circ} \mathrm{ARB} / 12 / 23$, sentence, 12 novembre 2016, $\S 130$ (la sentence se réfère à la doctrine et à la jurisprudence arbitrale d'investissement ayant reconnu le caractère coutumier des règles d'interprétation).

18. Comité ad hoc CIRDI, EDF SA et SAUR International SA c. Argentine, n ARB/03/23, décision sur l'annulation, 5 février 2016, $§ 66$. 
ou à des États non parties à cet instrument ${ }^{19}$, à plus forte raison s'ils s'en prévalent eux-mêmes ${ }^{20}$. Les articles 31 et suivants ont été tellement bien assimilés par la jurisprudence d'investissement que certains tribunaux en viennent à se référer à des précédents plutôt qu'à la convention de Vienne pour déterminer leur méthode interprétative, à l'exemple du tribunal de l'affaire Flemingo qui s'appuie sur la sentence Saluka pour établir que "the Tribunal must always bear in mind the terms of the Treaty under which it operates ${ }^{21}$...

La production arbitrale de 2016 est comme chaque année riche d'exemples de mise en œuvre des diverses techniques mentionnées par la convention de Vienne, et en premier lieu la "directive générale » 22 de l'article $31, \S 1^{\mathrm{er}}$, les moyens complémentaires de l'article 32 (travaux préparatoires, circonstances de conclusion du traité) s'avérant généralement peu déterminants ${ }^{23}$, quand l'article 33 (conciliation des versions linguistiques authentiques divergentes) est occasionnellement employé, notamment au sujet de la convention de Washington ${ }^{24}$. Les divergences linguistiques, assez fréquentes en pratique, peuvent également être résolues sans que l'aide de l'article 33 de la convention de Vienne soit décisive ${ }^{25}$, par exemple en conférant un poids supplémentaire à la version du traité correspondant à la langue utilisée dans les négociations ${ }^{26}$.

Le principe de bonne foi de l'interprétation est parfois mis en exergue ${ }^{27}$, qui doit selon l'article 31 conduire le tribunal à s'attacher en premier lieu au texte de la disposition à interpréter, en privilégiant le «sens ordinaire des termes » du traité 28

19. CPA (CNUDCI), Venezuela US, SRL c. Venezuela, $\mathrm{n}^{\circ} 2013-34$, sentence partielle sur la compétence, 26 juillet 2016, $\S \S 48-49$.

20. CIRDI, Crystallex International Corporation c. Venezuela, $\mathrm{n}^{\circ} \mathrm{ARB}(\mathrm{AF}) / 11 / 2$, sentence, 4 avril $2016, \S 537$.

21. CPA (CNUDCI), Flemingo Dutyfree Shop Private Ltd c. Pologne, sentence, 12 août 2016, § 295. La référence à la convention de Vienne intervient quelques paragraphes plus loin, au sujet de l'interprétation du terme investisseur ( $§ 319)$, alors que l'interprétation du terme « investissement » s'est bien faite auparavant selon le sens ordinaire des termes du traités.

22. Patrick DAILlier, Mathias ForTEAU et Alain Pellet, Droit international public (Nguyen Quoc Dinh), Paris, LGDJ, 2009, p. 290, n 170.

23. Voir CPA (CNUDCI), Philip Morris Asia Ltd c. Australie, $\mathrm{n}^{\circ}$ 2012-12, sentence sur la compétence et la recevabilité, 17 décembre $2015, \S \S 498$ et s. (où le tribunal ne tire des travaux préparatoires présentés aucune conclusion quant à l'intention des parties) ; CIRDI, Lundin Tunisia BV c. Tunisie, ${ }^{\circ}$ ARB/12/30, sentence, 22 décembre 2015, $\$ 144$ (référence aux rapport des administrateurs sur la Convention CIRDI); Comité ad hoc CIRDI, Total SA c. Argentine, $\mathrm{n}^{\circ}$ ARB/04/01, décision sur l'annulation, $1^{\text {er }}$ février 2016, $\S 166$; CIRDI, ConocoPhillips Petrozuata BV et al. c. Venezuela, $\mathrm{n}^{\circ} \mathrm{ARB} / 07 / 30$, décision sur la demande de réexamen de la décision du tribunal du 10 mars 2014, 9 février 2016, §§ 24, 26 ; Comité ad hoc CIRDI, EDF SA et SAUR International SA c. Argentine, $\mathrm{n}^{\circ} \mathrm{ARB} / 03 / 23$, décision sur l'annulation, 5 février 2016, $\S 142$. Pour une mise en œuvre confuse de l'art. 32, voir CIRDI, Tenaris SA et Talta-Trading e Marketing Sociedade Unipessoal Lda. c. Venezuela, n ARB/12/23, sentence, 29 janvier 2016, §§ 158, 170 (référence à des fins interprétatives à la pratique conventionnelle d'autres États et au droit interne).

24. CIRDI, Philip Morris Brands SARL, Philip Morris Products SA et Abal Hermanos SA c. Uruguay, $\mathrm{n}^{\circ} \mathrm{ARB} / 10 / 7$, décision sur la demande de modification de la sentence, 26 septembre 2016 , $\$ \S 16$-17; Comité ad hoc CIRDI, EDF SA et SAUR International SA c. Argentine, n ARB/03/23, décision sur l'annulation, 5 février 2016, $\$ 199$.

25. CIRDI, Saint-Gobain Performance Plastics Europe c. Venezuela, n ARB/12/13, décision sur la responsabilité et les principes de la réparation, 30 décembre $2016, \S 398$. Pour une affaire ayant soulevé de nombreux problèmes linguistiques résolus au moyen des articles 31 et s., voir CIRDI, İçkale İnşaat Limited Şirketi c. Turkménistan, ${ }^{\circ}$ ARB/10/24, sentence, 8 mars 2016, $\S 195$ et s.

26. CIRDI, Tenaris SA et Talta - Trading e Marketing Sociedade Unipessoal Lda. c. Venezuela, $\mathrm{n}^{\circ} \mathrm{ARB} / 12 / 23$, sentence, 12 novembre $2016, \S \S 124,814$.

27. CIRDI, Menzies Middle East and Africa SA et Aviation Handling Services International Ltd c. Sénégal, $\mathrm{n}^{\circ} \mathrm{ARB} / 15 / 21$, sentence, 5 août 2016, § 146 ; CIRDI, Tenaris SA et Talta - Trading e Marketing Sociedade Unipessoal Lda. c. Venezuela, ${ }^{\circ}$ ARB/12/23, sentence, 12 novembre 2016, § 140 ; CIRDI, SaintGobain Performance Plastics Europe c. Venezuela, ${ }^{\circ} \mathrm{ARB} / 12 / 13$, décision sur la responsabilité et les principes de la réparation, 30 décembre $2016, \S 420$.

28. CIRDI, Ampal-American Israel Corp. et al. c. Égypte, $\mathrm{n}^{\circ} \mathrm{ARB} / 12 / 11$, décision sur la compétence, $1^{\mathrm{er}}$ février 2016, $\S 144$ et s. ; Comité ad hoc CIRDI, Adem Dogan c. Turkménistan, n ARB/09/9, décision 
pris « dans leur contexte et à la lumière de son objet et de son but » ${ }^{29}$. Ces différents éléments doivent être appliqués simultanément ${ }^{30}$, ce qui devrait exclure qu'une interprétation soit fondée uniquement sur le sens littéral des mots ${ }^{31}$ (même si dans la pratique les tribunaux ne dépassent souvent pas cette étape) ${ }^{32}$ ou accorde aux objectifs poursuivis par les États parties une importance telle qu'elle dépasserait les termes employés dans la disposition à interpréter ${ }^{33}$. La validité d'une interprétation selon l'article $31, \S 1^{\mathrm{er}}$, a ainsi été déduite du fait qu'elle n'aboutissait pas à un résultat manifestement absurde ou déraisonnable au sens de l'article $32^{34}$. L'article $31, \S 1^{\mathrm{er}}$, est d'application générale, ce qui devrait exclure que certaines clauses fassent l'objet d'une interprétation plus restrictive, à l'exemple des clauses comportant des exceptions ${ }^{35}$.

Si l'on devait ne s'attarder que sur un exemple topique de mise en œuvre de l'article $31, \S 1^{\text {er }}$, on relèvera que, au stade de l'examen de sa compétence conditionnée par l'existence d'un investissement, le tribunal de l'affaire $R R E E F$ s'est référé à la définition très large donné par le traité sur la charte de l'énergie, avant de s'appuyer sur l'article 31 de la convention de Vienne pour rejeter tous autres «tests, criteria or guidelines beyond the terms, the context or the object and purpose of the ECT», et partant d'écarter les arguments tenant à la définition " objective » de l'investissement tirée par de nombreux tribunaux de l'interprétation de l'article 25 de la convention de Washington ${ }^{36}$. Il est vrai que cette dernière disposition, qui prévoit la compétence du CIRDI pour les différends « en relation avec un investissement ", ne définit pas ce dernier terme. C'est par un travail sophistiqué d'interprétation qu'à partir de ce silence tout un courant jurisprudentiel a développé une série de critères - variables selon les tribunaux ${ }^{37}$ - censés définir l’investissement

sur l'annulation, 15 janvier 2016, § 103 (recours au dictionnaire) ; CPA (CNUDCI), Flemingo Dutyfree Shop Private Ltd c. Pologne, sentence, 12 août 2016, $\$ 530$ (notant que le sens ordinaire doit être privilégié mais que le sens des termes " juste et équitable " varie en fonction des circonstances).

29. Confrontant l'interprétation selon le sens ordinaire des mots à l'objet et au but du traité applicable, voir CNUDCI, The Renco Group Inc. c. Pérou, UNCT/13/1, sentence partielle sur la compétence, 15 juillet 2016, $\S 84$ et s. ; CPA (CNUDCI), Mesa Power Group, LLC c. Canada, $\mathrm{n}^{\circ}$ 2012-17, sentence, 24 mars $2016, \S \S 231-233,295$ et $\mathrm{s}$. Notant que la prise en compte du préambule d'un traité, pertinente en matière d'interprétation selon le contexte, ne peut avoir pour effet de contredire les termes des dispositions substantielles, voir CPA (CNUDCI), Flemingo Dutyfree Shop Private Ltd c. Pologne, sentence, 12 août 2016, § 320.

30. CPA (CNUDCI), Murphy Exploration \& Production Company-International c. Équateur, sentence finale partielle, 6 mai 2016, § 158 .

31. Voir CPA, Windstream Energy LLC c. Canada, $\mathrm{n}^{\circ} 2013-22$, sentence, 27 septembre $2016, \S 357$ : le recours au dictionnaire pour définir les termes « juste " et " équitable » serait non seulement inutile car il faudrait interpréter les mots remplaçant les deux adjectifs, mais contre-productif car il pourrait aboutir à un nouveau standard non conforme au standard coutumier auquel renvoie la disposition de l'ALENA en cause.

32. Ex. : CIRDI, Ampal-American Israel Corp. et al. c. Égypte, $\mathrm{n}^{\circ} \mathrm{ARB} / 12 / 11$, décision sur la compétence, $1^{\mathrm{er}}$ février 2016, § 146 ; Ch. comm. Stockholm, Charanne BV et Construction Investment SARL c. Espagne, n $^{\circ}$ 062/2012, sentence, 21 janvier 2016, § 437 ; CIRDI, Crystallex International Corporation c. Venezuela, $\mathrm{n}^{\circ} \mathrm{ARB}(\mathrm{AF}) / 11 / 2$, sentence, 4 avril 2016, $\S 632$ et s., $\S 661$ et s.

33. Contra voir l'opinion dissidente de l'arbitre (nommé par le demandeur) dans l'affaire CIRDI, Vincent J. Ryan, Schooner Capital LLC and Atlantic Investment Partners LLC c. Pologne, $\mathrm{n}^{\circ} \mathrm{ARB}(\mathrm{AF}) / 11 / 13$, op. diss. Orrego Vicuña jointe à la sentence du 24 novembre 2015, § 6, pour qui l'interprétation des «questions fiscales » exclues de la compétence du tribunal doit tenir compte de l'objectif global du traité qui est la promotion de l'investissement, objectif que remet en cause l'interprétation par trop textuelle des arbitres majoritaires.

34. CIRDI, Saint-Gobain Performance Plastics Europe c. Venezuela, n ARB/12/13, décision sur la responsabilité et les principes de la réparation, 30 décembre $2016, \S \S 420,424$.

35. CPA (CNUDCI), Mesa Power Group, LLC c. Canada, n 2012-17, sentence, 24 mars 2016, § 405.

36. CIRDI, RREEF Infrastructure (GP) Limited et RREEF Pan-European Infrastructure Two Lux SARL c. Espagne, n ARB/13/30, décision sur la compétence, 6 juin 2016, $\S 157$.

37. Voir par ex. CIRDI, Joseph Houben c. Burundi, $\mathrm{n}^{\circ}$ ARB/13/7, sentence, 12 janvier 2016, §§ 112 et s. ; CIRDI, Vestey Group Limited c. Venezuela, n ARB/06/4, sentence, 15 avril 2016, § 187. 
(le «Salini test » dans le jargon) ${ }^{38}$, avec lequel la décision RREEF s'inscrit en porteà-faux en revenant au b.a.-ba de l'interprétation : l'article $31^{39}$. Cette décision ne doit pas occulter le fait que la jurisprudence des tribunaux d'investissement est (sur)mobilisée par les parties dans leurs argumentaires et par les tribunaux dans leurs décisions. Ce phénomène s'explique par le fait que de nombreux traités d'investissement comportent des dispositions ou emploient des termes identiques ou très proches. Dès lors, il n'est guère surprenant que l'interprétation des tribunaux repose très largement ${ }^{40}$, voire exclusivement ${ }^{41}$, sur les décisions arbitrales antérieures qui en ont déterminé la portée, la référence éventuelle à l'article 31 de la convention de Vienne n'étant alors que purement cosmétique ${ }^{42}$. Ce recours au précédent trouve néanmoins rapidement ses limites dans l'existence de courants contraires sur des questions pourtant déterminantes, à l'image de la définition de l'investissement. L'interprétation revient alors souvent à choisir un courant plutôt qu'un autre... ou à revenir aux techniques de la convention de Vienne.

À côté du « contexte interne », le « contexte externe » ${ }^{43}$ du traité (art. $31, \S 3$ ) est susceptible d'être mobilisé dans le cadre du processus interprétatif. Les tribunaux arbitraux sont ainsi ponctuellement invités ou conduits à tenir compte dans leur interprétation des traités d'investissement d'accords ultérieurs intervenus entre les parties (art. 31, § 3, a) ${ }^{44}$ ou de pratiques ultérieurement suivies par elles (art. 31, $\S 3, \mathrm{~b})^{45}$. Mais les interprétations les plus stimulantes restent celles résultant de la prise en compte « [d]e toute règle pertinente de droit international applicable dans les relations entre les parties » (article 31, § 3, c).

38. Voir A. DE NANTeuil, Droit international de l'investissement, Paris, Pedone, 2017, 2éd., pp. 178 et $\mathrm{s}$.

39. S'agissant de l'article 25 de la convention de Washington, le tribunal ne s'étend pas sur sa démarche interprétative. Il se contente d'affirmer que " $[t]$ here is no reason to place any such test, set of criteria or guidelines on the language of Article 25 of the ICSID Convention " (\$157).

40. Ex. : CPA (CNUDCI), Flemingo Dutyfree Shop Private Ltd c. Pologne, sentence, 12 août 2016, $\S 529$ et s.

41. Ex. : CIRDI, Crystallex International Corporation c. Venezuela, $\mathrm{n}^{\circ} \mathrm{ARB}(\mathrm{AF}) / 11 / 2$, sentence, 4 avril $2016, \S \S 530$ et s. (au sujet de l'expression « conformément au droit international » figurant dans la clause sur le traitement juste et équitable); CIRDI, Fábrica de Vidrios La Andes, CA et Owens-Illinois de Venezuela, $C A$ c. Venezuela, $\mathrm{n}^{\circ} \mathrm{ARB} / 12 / 21$, décision sur la demande de récusation de L. Yves Fortier, arbitre, 28 mars 2016, § 28 (au sujet de l'article 14 de la convention de Washington relatif à l'indépendance des arbitres, exprimée différemment selon les versions française, anglaise et espagnole - toutes trois authentiques - de la convention).

42. CIRDI, Vestey Group Limited c. Venezuela, ${ }^{\circ}$ ARB/06/4, sentence, 15 avril 2016, $§ 187$.

43. Jean-Marc SOREL, "Article 31 ", in Olivier CORTEN et Pierre KLEIN (dir.), Les Conventions de Vienne sur le droit des traités. Commentaire article par article, Bruxelles, Bruylant, 2006, p. 1319.

44. Voir CPA, Windstream Energy LLC c. Canada, n 2013-22, sentence, 27 septembre 2016, §§ 348, 356 (alignement du tribunal sur les notes d'interprétation de la Commission du libre-échange de l'ALENA sur l'art. 1105, la mention d'un traitement conforme au droit international qu'il contient renvoyant selon elle au droit coutumier - il y a là une forme d'interprétation authentique par un organe politique, cette pratique étant promise à un bel avenir si l'on en croit les accords récents conclus par l'Union européenne avec le Canada et avec Singapour). Voir aussi CPA (CNUDCI), Mesa Power Group, LLC c. Canada, $\mathrm{n}^{\circ} 2012$ 17, sentence, 24 mars 2016, §§ 479 et s. ; CNUDCI, The Renco Group Inc. c. Pérou, UNCT/13/1, sentence partielle sur la compétence, 15 juillet 2016, $\S \S 156-157$ (où le Pérou invoque un accord ultérieur entre cet État et les États-Unis d'Amérique, non pris en considération par le tribunal qui estime n'être pas lié par les positions des États dans son interprétation du traité).

45. CIRDI, Menzies Middle East and Africa SA et Aviation Handling Services International Ltd c. Sénégal, $\mathrm{n}^{\circ} \mathrm{ARB} / 15 / 21$, sentence, 5 août $2016, \S 150$ (prise en compte de la pratique conventionnelle ultérieure des parties à l'Accord général sur le commerce des services : "Si les Etats membres de l'AGCS ont continué à conclure, et, en fait, ont intensifié leurs efforts de conclure, des TBIs offrant aux investisseurs la possibilité de recourir à l'arbitrage, c'est parce que ces mêmes États n'ont pas entendu offrir aux fournisseurs de services, via l'AGCS, l'accès à l'arbitrage international »). 
Elles se font discrètes lorsqu'elles prennent la forme de références inopinées à la jurisprudence internationale " générale " 46 voire à celle propre à d'autres spécialités du droit international ${ }^{47}$ aux fins d'interpréter les dispositions des traités d'investissement ou de la convention de Washington. Elles sont bien plus spectaculaires lorsqu'elles permettent d'importer dans le contentieux d'investissement des normes matérielles de nature à rééquilibrer la répartition, qui dans les traités ne l'est pas, des droits et des obligations entre États et investisseurs protégés. Dans l'affaire Philip Morris c. Uruguay, l'interprétation du traité Suisse/Uruguay par ce moyen a ainsi justifié l'application de règles coutumières en matière de police powers de l'État ${ }^{48}$. Le tribunal de l'affaire Urbaser s'est également appuyé sur l'article $31, \S 3, \mathrm{c}$, pour considérer que « [t]he BIT cannot be interpreted and applied in a vacuum "; qu'il " has to be construed in harmony with other rules of international law of which it forms part, including those relating to human rights " 49 . Cette « approche » est selon le tribunal reflétée dans la clause du traité sur le droit applicable qui, prévoyant l'application du droit international, atteste que «the BIT is not framed in isolation, but placed in the overall system of international law ${ }^{50}$. Mais en l'occurrence, l'interprétation systémique n'est pas la seule employée pour justifier l'application du droit international des droits de l'homme au différend. Quelques paragraphes auparavant, le tribunal était en effet parvenu au même résultat au moyen du principe d'effet utile, tiré du principe d'interprétation de bonne foi des traités (art. 31 de la convention de Vienne) ${ }^{51}$. La clause sur le droit applicable prévoyait en effet que le tribunal devait fonder sa décision sur le traité d'investissement et sur les principes généraux de droit international. Or, pour le tribunal cette dernière référence "would be meaningless if the position would be retained that the BIT is to be construed as an isolated set of rules of international law for the sole purpose of protecting investments through rights exclusively granted to investors $" 52$.

46. Ex. : CIRDI, Saint-Gobain Performance Plastics Europe c. Venezuela, $\mathrm{n}^{\circ} \mathrm{ARB} / 12 / 13$, décision sur la responsabilité et les principes de la réparation, 30 décembre 2016, § 394 (interprétation du terme " mesure " par la CIJ dans l'affaire Compétence en matière de pêcheries) ; CIRDI, RREEF Infrastructure (GP) Limited et RREEF Pan-European Infrastructure Two Lux SARL c. Espagne, $\mathrm{n}^{\circ} \mathrm{ARB} / 13 / 30$, décision sur la compétence, 6 juin 2016, § 119 (jurisprudence de la CIJ sur les droits des actionnaires).

47. Ex. : CPA (CNUDCI), Mesa Power Group, LLC c. Canada, $\mathrm{n}^{\circ}$ 2012-17, sentence, 24 mars 2016, $\S \S 411$ (jurisprudence de l'OMC sur le sens du terme " procurement ») ; CNUDCI, The Renco Group Inc. c. Pérou, UNCT/13/1, sentence partielle sur la compétence, 15 juillet 2016, § 164 (jurisprudence de la CEDH sur l'autonomie de la clause compromissoire).

48. CIRDI, Philip Morris Brands SARL, Philip Morris Products SA c. Uruguay, $\mathrm{n}^{\circ} \mathrm{ARB} / 10 / 7$, sentence, 8 juillet $2016, \S \S 290$ et $\mathrm{s}$. Voir infra, B (Coutume).

49. CIRDI, Urbaser SA et Consorcio de Aguas Bilbao Bizkaia, Bilbao Biskaia Ur Partzuergoa $c$. Argentine, $\mathrm{n}^{\circ} \mathrm{ARB} / 07 / 26$, sentence, 8 décembre 2016, $\S 1200$.

50. Ibid., § 1201 .

51. Sur le principe d'effet utile, voir aussi CIRDI, Tenaris SA et Talta-Trading e Marketing Sociedade Unipessoal Lda. c. Venezuela, $\mathrm{n}^{\circ} \mathrm{ARB} / 12 / 23$, sentence, 12 novembre 2016, $\S 1$ 137, 189 ; CIRDI, Spence International Investments, LLC et al. c. Costa Rica, $\mathrm{n}^{\circ} \mathrm{UNCT} / 13 / 2$, sentence intérimaire, 25 octobre 2016 , $\S 217$; CIRDI, Saint-Gobain Performance Plastics Europe c. Venezuela, n ARB/12/13, décision sur la responsabilité et les principes de la réparation, 30 décembre 2016, §424; CPA (CNUDCI), Mesa Power Group, LLC c. Canada, $\mathrm{n}^{\circ}$ 2012-17, sentence, 24 mars 2016, §§ 299, 403 ; CIRDI, Tenaris SA et TaltaTrading e Marketing Sociedade Unipessoal Lda. c. Venezuela, $\mathrm{n}^{\circ}$ ARB/12/23, sentence, 29 janvier 2016, $\S \S 148$ et s. ; CIRDI, İçkale İnşaat Limited Şirketi c. Turkménistan, n ARB/10/24, sentence, 8 mars 2016, $\S 329$; Trib. ad hoc (CNUDCI), Oxus Gold plc c. Ouzbékistan, sentence finale, 17 décembre 2015, § 354 (où le tribunal, après l'avoir mis en œuvre, constate que «the effet utile principle is an important element of interpretation of legal instruments "; voir aussi § 369). Contra, voir Comité ad hoc CIRDI, EDF SA et SAUR International SA c. Argentine, n ${ }^{\circ}$ ARB/03/23, décision sur l'annulation, 5 février $2016, \S 312$, où le comité ad hoc rejette l'argument de l'Argentine implicitement fondé sur le principe d'effet utile en constatant : "It is by no means unusual for the parties to a treaty to include, ex abundante cautela, a provision which may not be strictly necessary".

52. CIRDI, Urbaser SA c. Argentine, § 1189. 
Ces approches qui mêlent de manière difficilement extirpable techniques d'interprétation et question de droit applicable ${ }^{53}$ pourront réjouir ceux que le fameux déséquilibre du contentieux d'investissement - qui repose avant tout sur le déséquilibre des traités qui le génèrent - tracasse à juste titre. L'interprétation systémique, en ce qu'elle est un facteur de défragmentation du droit international ${ }^{54}$, est par ailleurs vue sous un jour favorable par la doctrine internationaliste, attachée à l'unité de «sa » discipline. Elle interroge néanmoins sur les risques de « débordement » si elle devait être employée inconsidérément par des tribunaux arbitraux dont on sait qu'ils cèdent parfois à la fantaisie dans leurs raisonnements... Il ne faudrait pas qu'ils oublient, par exemple, que dans le cadre de l'article 31, § 3, c seules les règles de droit international « applicable[s] entre les parties » peuvent être employées à des fins interprétatives ${ }^{55}$, et qu'il leur revient d'en « tenir compte » et non pas de priver d'effet la disposition conventionnelle interprétée. On a aussi pu soutenir que l'interprétation systémique introduit de la confusion dans l'interprétation en ce qu'elle s'éloigne de la primauté du texte tirée de l'article $31, \S 1^{\mathrm{er}}$, de la convention de Vienne ${ }^{56}$. Or, il ne faudrait pas négliger le fait que, du côté des investisseurs comme des États, " a prudential use of interpretation rules by investment tribunals helps to strengthen confidence in the arbitration system and ensure legal certainty ${ }^{57}$.

S'agissant des problèmes d'articulation entre les normes internationales immédiatement applicables et celles d'origine exogène, on prendra note avec intérêt du principe d'interprétation harmonieuse dégagé dans la décision $R R E E F$, à rebours de la décision Electrabel dans laquelle les arbitres avaient considéré qu'il n'existait pas de "general principle of international law compelling the harmonious interpretation of different treaties ", se contentant d'y voir un simple résultat souhaitable ( desirable outcome ») ${ }^{58}$. Bien au contraire, pour le tribunal de l'affaire RREEF, des traités même inégalement applicables à un différend devraient être interprétés de manière à ne pas se contredire ${ }^{59}$. Même si en l'espèce étaient en cause les rapports entre le traité de la charte de l'énergie et le droit de l'Union européenne, on perçoit les potentialités d'un tel principe dans d'autres contextes, notamment au sujet de l'articulation entre les traités d'investissement et la convention de Washington ${ }^{60}$.

53. Voir Hervé AsCENSIO, «Article 31 of the Vienna Convention on the Law of Treaties and International Investment Law », ICSID Review, 2016, vol. 31, n² 2, p. 308. Sur les questions de droit applicable, voir infra, III, B.

54. Rapport du Groupe d'étude de la CDI, établi sous sa forme définitive par Martii KosKENNIEMI, «Fragmentation du droit international : difficultés découlant de la diversification et de l'expansion du droit international ", A/CN.4/L.682, 13 avril 2006, pp. 225 et s., $\$ \$ 410$ et s.

55. On notera à cet égard le subterfuge du tribunal de l'affaire Philip Morris qui a consisté à faire de la convention antitabac de l'OMS un " point de référence ", notamment pour apprécier le caractère raisonnable des mesures prises par l'Uruguay, alors que la convention n'était pas applicable dans les relations entre les parties au traité d'investissement, la Suisse (État de nationalité de l'investisseur) n'étant pas partie à cet instrument (CIRDI, Philip Morris c. Uruguay, ${ }^{\circ}$ ARB/10/7, sentence du 8 juillet 2016, $\S 401$ ). Voir aussi CIRDI, Saint-Gobain Performance Plastics Europe c. Venezuela, n ARB/12/13, décision sur la responsabilité et les principes de la réparation, 30 décembre 2016 , $\$ 489$, où l'Accord économique et commercial global (AECG/CETA) - non entré en vigueur et ne concernant pas, en tout état de cause, le Venezuela - est employé aux fins d'interprétation de la clause sur le traitement juste et équitable (le tribunal note toutefois que le défendeur s'y est lui-même référé).

56. Hervé ASCENSIO, op. cit., p. 387.

57. Laurence BOISSON DE CHAZOURNES, "Rules of Interpretation and Investment Arbitration ", in Meg KINNEAR et al. (ed.), Building International Investment Law : The First 50 years of ICSID, Alphen aan den Rijn, ICSID/Wolters Kluwer, 2016, p. 17.

58. CIRDI, RREEF Infrastructure (GP) Limited et RREEF Pan-European Infrastructure Two Lux $S A R L$ c. Espagne, n $\mathrm{ARB} / 13 / 30$, décision sur la compétence, 6 juin 2016, $\$ 76$.

59. Ibid.

60. Voir à cet égard CIRDI, Ampal-American Israel Corp. et al. c. Égypte, ${ }^{\circ} \mathrm{ARB} / 12 / 11$, décision sur la compétence, $1^{\text {er }}$ février 2016, $§ 169$, où le tribunal indique - sans le justifier - que le traité Égypte/ États-Unis doit être interprété à la lumière de la convention de Washington. 
Reste que la consécration de ce nouveau «cannon of interpretation » relève plus de l'affirmation que de la démonstration ${ }^{61}$; il ressortit davantage à la lex ferenda qu'à la lex lata interprétative. Il témoigne néanmoins du dynamisme de la jurisprudence arbitrale d'investissement dans la mise en œuvre et peut-être l'évolution du droit international de l'interprétation.

\section{Autres questions de droit des traités}

Hormis les sempiternelles questions d'interprétation, peu de sujets intéressant le droit international général des traités émergent de la production arbitrale de 2016. On relèvera pour mémoire que le principe de non-rétroactivité des traités exprimé à l'article 28 de la convention de Vienne de 1969 a été mobilisé en lien avec la compétence ratione temporis des tribunaux ${ }^{62}$, sans qu'il fasse obstacle à ce qu'un tribunal prenne en considération des comportement antérieurs à l'entrée en vigueur d'un traité "for purposes of determining whether there was a post-entry into force breach of a justiciable obligation ${ }^{63}$. De même, la dénonciation de la Convention de Washington par le Venezuela a donné lieu à plusieurs décisions, qui intéressent toutefois moins le droit des traités que le droit du contentieux, à travers la question du consentement à l'arbitrage ${ }^{64}$. L'attention se portera ici sur deux questions qui témoignent du fait que le droit international des rapports interétatiques classiques ne saurait être transposé sans prudence aux rapports transnationaux État/investisseur : celle des réserves (a) et celle de l'ordre public international (b).

\section{a) Réserves}

L'affaire Renco a donné lieu à une curieuse analogie entre les réserves du droit des traités et celles qu'un investisseur peut émettre dans le cadre de la procédure de règlement des litiges avec l'État d'accueil de son investissement. En l'espèce, la clause de règlement des différends du traité États-Unis/Pérou prévoyait comme condition à la compétence du tribunal arbitral que l'investisseur renonçât par écrit à son droit de poursuivre l'État devant les juridictions internes ${ }^{65}$. L'acceptation de l'offre d'arbitrage de l'État par Renco comportait une renonciation («waiver») en ce sens, qui se terminait néanmoins par une phrase selon laquelle l'investisseur se réservait le droit de soumettre le fond de sa réclamation à un autre juge si le tribunal saisi devait rejeter sa demande à titre préliminaire.

Le tribunal a alors interrogé les parties sur la « divisibilité " (severability) de cette dernière phrase jugée incompatible avec les prescriptions du traité. Pour attester l'existence d'un principe de « divisibilité » de nature à sauver sa déclaration de renonciation, Renco s'est alors appuyé sur le droit interétatique des réserves, en se référant notamment à la jurisprudence de la Cour européenne des droits de

61. Le tribunal indique simplement que ce principe d'interprétation ne diffère pas de la position de la Cour de Justice de l'Union européenne qui, face à deux interprétations possibles d'une norme de droit dérivé, choisit celle qui est conforme aux traités (CJCE, Commission européenne c. Allemagne, arrêt du 10 septembre 1996, aff. C-61/94, Rec., I-4020-4021, § 52).

62. CPA (CNUDCI), Mesa Power Group, LLC c. Canada, $\mathrm{n}^{\circ} 2012-17$, sentence, 24 mars 2016, § 325 ; CIRDI, Spence International Investments, LLC et al. c. Costa Rica, $\mathrm{n}^{\circ} \mathrm{UNCT} / 13 / 2$, sentence intérimaire, 25 octobre $2016, \S 215$. Sur la distinction conceptuelle, trop souvent occultée, entre compétence temporelle du tribunal et applicabilité du traité dans le temps, voir le comm. d'Arnaud DE NANTEUIL, in Gazette du Palais - Les Cahiers de l'arbitrage, 2009, n 4, pp. 33-37.

63. Spence International Investments, § 216. Voir infra, II, A, 2 (fait composite).

64. Voir infra, III, A, 2.

65. Art. 10.18(2) du traité. 
l'homme et au Guide de la pratique sur les réserves aux traités adopté en 2011 par la Commission du droit international des Nations Unies ${ }^{66}$.

Le tribunal a néanmoins refusé de consacrer un principe général de divisibilité ${ }^{67}$. Il a noté de surcroît que "States are entitled, by virtue of their sovereignty, to apply such reservations to treaties as are permitted by Article 19 of the VCLT. Investors have no equivalent power to insert reservations into their acceptances of a State's standing offer to arbitrate under an investment treaty " ${ }^{6}$. L'analogie était en effet biaisée dès le départ. Il faut comprendre que la distinction entre la " réserve " de l'État et celle de l'investisseur ne porte pas sur la fonction même de l'acte : dans les deux cas il vise à " exclure ou à modifier l'effet juridique » 69 d'un engagement. Elle concerne son objet : dans le premier cas, elle porte sur la clause d'un traité ; dans le second sur la déclaration de renonciation. D'une part, la réserve est régie par la clause du traité relative aux réserves ou à défaut par le droit international général ${ }^{70}$; d'autre part, la réserve de l'investisseur doit être en tous points conforme, sous peine d'invalidité, aux conditions fixées par la clause de règlement des différends du traité d'investissement. Il s'agit de conditions du consentement de l'État à l'arbitrage dont l'irrespect entraîne l'incompétence du tribunal. La comparaison avec les « réserves » émises par les États dans leurs déclarations de juridiction obligatoire devant la CIJ n'est guère plus pertinente. Dans un cas, l'État souverain consent à la juridiction de la Cour moyennant des restrictions permises par l'article 36 du Statut de la CIJ ; dans l'autre, un investisseur renonce à saisir le juge interne moyennant une réserve contraire à la clause de règlement des différends du traité applicable. Il y avait là un mélange des genres suffisant pour exclure la transposition d'un éventuel principe de divisibilité.

\section{b) Jus cogens et ordre public international}

La nullité des traités d'investissement pour conflit avec une norme impérative du droit international général, au sens de l'article 53 de la convention de Vienne, ne fait pas partie - et c'est heureux ! - des questions débattues devant les tribunaux d'investissement. Pour autant, les décisions arbitrales qui se réfèrent au jus cogens tendent à se multiplier ${ }^{71}$. Il ne s'agit ici que d'un bref obiter dictum, indiquant que dans le cadre du litige soumis au tribunal, le traité sur la charte de l'énergie, à l'origine de sa compétence et constituant ainsi son instrument « constitutionnel », prévaut sur le droit de l'Union européenne comme sur toute autre norme « apart from those of ius cogens " 72 . Là, tout à sa démonstration selon laquelle, conformément à l'article 31, § 3, c, de la convention de Vienne, un traité d'investissement

66. CNUDCI, The Renco Group Inc. c. Pérou, UNCT/13/1, sentence partielle sur la compétence, 15 juillet $2016, \S 164$, où il est fait référence au commentaire de la directive 4.5.3 dans lequel la CDI se rallie "à l'idée d'une présomption, réfragable, selon laquelle, en l'absence d'intention contraire de son auteur, le traité s'applique à l'État ou à l'organisation internationale auteur d'une réserve non valide nonobstant cette réserve » (A/66/10/Add.1; p. 565, § 40).

67. Ibid., § 170. Voir infra, C.

68. Ibid., § 171.

69. Directives 1.1 du Guide de la pratique sur les réserves aux traités.

70. Voir les Directives 3.1 et s. du Guide de la pratique de la CDI.

71. Voir par ex. CIRDI, Phoenix Action, Ltd. c. République tchèque, $\mathrm{n}^{\circ} \mathrm{ARB} / 06 / 5$, sentence du 15 avril 2009, § 77 (cette chronique, cet Annuaire, 2009, p. 687) ; CIRDI, EDF International SA, SAUR International SA and León Participaciones Argentinas SA c. Argentine, $\mathrm{n}^{\circ}$ ARB/03/23, sentence du 11 juin 2012 , $\S \S 911-914$ (cette chronique, cet Annuaire, 2012, p. 648). Voir Valentina VADI, "Jus cogens in International Investment Law and Arbitration ", Netherlands Yearbook of International Law, 2015, pp. 357-388.

72. CIRDI, RREEF Infrastructure (GP) Limited et RREEF Pan-European Infrastructure Two Lux SARL c. Espagne, n ARB/13/30, décision sur la compétence, 6 juin 2016, $§ 75$. 

«is not framed in isolation, but placed in the overall system of international law ${ }^{73}$,
un autre tribunal prend la peine de noter :

«Another illustration is given by peremptory norms of general international law (ius cogens) to the extent they may be of interest in an investment matter. If so, such norms must certainly prevail over any contrary provision of the BIT, as per the express statement in Article 53 of the Vienna Convention. " ${ }^{74}$

Ces références au jus cogens sont moins opérationnelles que publicitaires. Si elles ne produisent en effet aucune espèce de conséquence palpable dans les affaires jugées, elles constituent de discrets petits coups de pouce à la promotion et à la consolidation de la « différenciation hiérarchique des normes » 75 en droit international à travers la reconnaissance d'un ordre public propre à cet ordre juridique.

Ce dernier ne doit toutefois pas être confondu avec l'" ordre public international » invoqué par les arbitres de l'affaire Getma pour examiner leur compétence nonobstant le principe res judicata ou le caractère tardif des arguments soulevés ${ }^{76}$. Dans cette affaire, les arbitres se sont appuyés sur l'ordre public « réellement international » ou «transnational » initialement à l'œuvre dans l'arbitrage commercial international 77 et qui s'est diffusé ensuite dans l'arbitrage d'investissement ${ }^{78}$. On peut certes s'employer à inscrire cet ordre public « dans le giron du droit international public (coutumier) " 79 . Reste que l'interdiction de la corruption, que les arbitres des affaires World Duty Free ou Getma font relever de l'ordre public international/transnational, ne se situe vraisemblablement pas au même niveau d'impérativité que l'interdiction du génocide ou de l'esclavage. L'ordre public international/ transnational demeure à ce jour un concept distinct du jus cogens du droit international public tant dans son contenu (celui du jus cogens semble plus resserré) que dans ses fonctions. Cela étant, il n'est pas dit que les deux concepts ne finiront pas par se rejoindre ${ }^{80}$, à travers l'édification d'un ordre public réellement transnational dont l'arbitrage d'investissement serait à n'en pas douter l'un des artisans.

73. CIRDI, Urbaser SA et Consorcio de Aguas Bilbao Bizkaia, Bilbao Biskaia Ur Partzuergoa $c$. Argentine, $\mathrm{n}^{\circ} \mathrm{ARB} / 07 / 26$, sentence, 8 décembre 2016, $\S 1201$.

74. Ibid., § 1203 .

75. Voir Jean ComBACAU, « Le droit international : bric-à-brac ou système ? ", Arch. philo. dr., 1986, vol. 31, p. 10 : «La promotion du jus cogens tend à introduire dans un système fondé sur l'indifférenciation hiérarchique des normes une notion d'ordre public calquée sur celle qui assure en droit interne l'encadrement des actes juridiques privés par un droit objectif imposé par l'État ».

76. CIRDI, Getma International, NCT Necotrans, Getma International Investments, NCT Infrastructure \& Logistique c. Guinée, $\mathrm{n}^{\circ} \mathrm{ARB} / 11 / 29$, sentence, 16 août 2016, §§ 178-180. Voir en particulier $\S 178$ : "L'ordre public international exige que ce Tribunal arbitral s'exprime sur l'allégation grave de corruption, même si un autre tribunal s'est déjà penché sur la question, quand de nouvelles preuves non analysées par le premier tribunal sont produites".

77. Voir Pierre LALIVE, « Ordre public transnational (réellement international) et arbitrage international », Rev. Arb., 1986, n³ 3 , pp. 329-374.

78. CIRDI, World Duty Free Company c. Kenya, n ABR/00/7, sentence, 4 octobre 2006, § 157, où le tribunal conclut que " $[i] n$ light of domestic laws and international conventions relating to corruption, and in light of the decisions taken in this matter by courts and arbitral tribunals, this Tribunal is convinced that bribery is contrary to the international public policy of most, if not all, States or, to use another formula, to transnational public policy".

79. Mathias FoRTEAU, «L'ordre public 'transnational' ou 'réellement international' - L'ordre public international face à l'enchevêtrement croissant du droit international privé et du droit international public », JDI (Clunet), 2011/1, §58.

80. Implicitement en ce sens, voir Mathias FORTEAU, « La contribution au développement du droit international général de la jurisprudence arbitrale relative aux investissements étrangers ", Brazilian Yearbook of International Law, vol. IV, 2009/1, pp. 23 et s. 


\section{B. Coutume}

Dans un contentieux d'investissement fondé dans une très large mesure sur des traités, la source coutumière demeure paradoxalement fort présente. C'est que, d'une part, le droit coutumier des investissements s'épanouit parallèlement aux règles conventionnelles, dont ces dernières se nourrissent et qu'elles sont susceptibles d'alimenter par retour de balancier ${ }^{81}$, et que, d'autre part, les traités d'investissement ne sont pas autosuffisants : les tribunaux arbitraux, pour trancher les litiges qui leur sont soumis, doivent rechercher des solutions dans le droit international général (droit des traités ${ }^{82}$ et droit de la responsabilité ${ }^{83}$ notamment). Partant, l'internationaliste généraliste souhaitant rester averti de l'état du droit international coutumier et déceler ses évolutions serait bien imprudent de négliger l'apport des décisions des tribunaux d'investissement. La méthode suivie par les arbitres pour déterminer le droit coutumier devrait tout autant, sinon plus, retenir son attention, ainsi que plusieurs décisions de 2016 tendent à l'indiquer.

S'agissant, tout d'abord, de la « charge » de la détermination de la coutume, le tribunal de l'affaire Windstream Energy a estimé qu'il revenait à la partie alléguant l'existence d'une règle coutumière d'en prouver l'existence ; qu'en revanche si les deux parties sont en désaccord sur le contenu d'une règle existante «it is for each Party to support its position as to the content of the rule with appropriate legal authorities and evidence " ${ }^{4}$. La première partie de la proposition pourra étonner, en ce qu'elle laisse entendre que le tribunal ne pourrait constater l'existence d'une règle coutumière si la partie qui s'en prévaut ne s'est pas délestée du fardeau de la preuve. Une telle affirmation heurte le principe iura novit curia ${ }^{85}$, que le tribunal met d'ailleurs implicitement en œuvre au sujet de la deuxième proposition ${ }^{86}$.

La question des moyens mis en œuvre par les tribunaux arbitraux pour déterminer le droit coutumier mérite tout autant une attention critique, qui plus est dans le contexte des travaux de la CDI à ce sujet ${ }^{87}$.

81. Voir Gérard CAHIN, «Droit international coutumier et traités d'investissement - Aspects méthodologiques ", in Mélanges offerts à Charles Leben. Droit international et culture juridique, Paris, Pedone, 2015, pp. 17-44.

82. Voir supra, A.

83. Voir par ex. CIRDI, Joseph Houben c. Burundi, $\mathrm{n}^{\circ}$ ARB/13/7, sentence, 12 janvier 2016, § 218 (le traité applicable ne définissant pas de standard d'évaluation du préjudice, celui-ci « doit par suite être évalué conformément au droit international coutumier "). Voir infra, II.

84. CPA, Windstream Energy LLC c. Canada, n 2013-22, sentence, 27 septembre 2016, § 350.

85. Voir CIJ, Compétence en matière de pêcheries (République fédérale d'Allemagne c. Islande), arrêt du 25 juillet 1974, Rec. CIJ, 1974, p. $181 \S 18$ : « La Cour ayant pour fonction de déterminer et d'appliquer le droit dans les circonstances de chaque espèce, la charge d'établir ou de prouver les règles de droit international ne saurait être imposée à l'une ou l'autre Partie, car le droit ressortit au domaine de la connaissance judiciaire de la Cour ".

86. CPA, Windstream Energy LLC c. Canada, § 350. Le tribunal semble reprendre à son compte la position du Canada, non contestée par le demandeur, selon laquelle « if the Tribunal considers that there are issues or questions that neither Party has fully or properly addressed, or if it wishes to refer to legal authorities other than those cited by the Parties, it should draw the Parties'attention to those issues, questions and authorities and solicit the Parties' views thereon". Voir CIRDI, Churchill Mining PLC et Planet Mining Pty Ltd c. Indonésie, $\mathrm{n}^{\circ} \mathrm{ARB} / 12 / 14$ et 12/40, sentence, 6 décembre 2016, § 236 ( "The principle iura novit curia - or better, iura novit arbiter - allows the Tribunal to form its own opinion of the meaning of the law, provided that it does not surprise the Parties with a legal theory that was not subject to debate and that the Parties could not anticipate ").

87. En août 2016, la Commission du droit international a adopté, en première lecture, un ensemble complet de seize projets de conclusions sur le sujet " Détermination du droit international coutumier " (A/71/10, chapitre V). 
Pour démontrer le caractère coutumier de la doctrine des "police powers " de l'État ${ }^{88}$, le tribunal arbitral de l'affaire Philip Morris c. Uruguay s'est référé à de nombreuses normes de soft law : le projet de convention de Harvard relatif à la responsabilité internationale des États à raison des dommages causés sur leur territoire à la personne ou aux biens des étrangers (1960), le Third Restatement of the Foreign Relations Law (1987) de l'American Law Institute, un "working paper » de l'OCDE sur l'expropriation de 2004, auxquels s'ajoute dans une période plus récente " a range of investment decisions [that] have contributed to develop the scope, content and conditions of the State's police powers doctrine, anchoring it in international law 89 . Parachevant sa démonstration, le tribunal se réfère enfin à la pratique conventionnelle récente de certains États (modèles de traités d'investissement des États-Unis et du Canada, ainsi que les accords commerciaux de l'Union européenne avec le Canada et avec Singapour), dont il déduit que les dispositions pertinentes "whether or not introduced ex abundanti cautela, reflect the position under general international law ${ }^{90}$. Cette démonstration de la norme coutumière à partir d'instruments soft ${ }^{91}$, de décisions arbitrales et d'échantillons conventionnels pourra paraître insuffisante pour établir l'existence d'une pratique généralisée acceptée comme étant le droit... Elle semble pourtant solidement argumentée si on la compare à d'autres décisions arbitrales rendues également en 2016.

Ainsi, la démarche purement jurisprudentielle suivie par le tribunal arbitral de l'affaire Saint-Gobain pour établir le caractère coutumier des intérêts composés - que la CDI avait écartés de son travail de codification du droit de la responsabilité 92 - ne manquera pas d'étonner. Les arbitres commencent en effet par relever que la sentence Autopista c. Venezuela de 2003 avait jugé que « compound interest was not an established principle under, and therefore not required by, international $l a w »{ }^{93}$. Puis ils considèrent qu'il est approprié de réévaluer cette conclusion. Plutôt que de suivre alors la méthodologie classique d'identification du droit coutumier le tribunal ne s'appuie que sur des décisions rendues par d'autres tribunaux. Le fait que certains tribunaux n'aient accordé que des intérêts simples - et donc que la pratique arbitrale en la matière n'est pas générale - n'est pas suffisant pour ébranler les certitudes du tribunal : "it appears that this was either due to the particular circumstances prevailing in the respective case or not supported by any reasoning for the tribunal's decision " ${ }^{94}$. C'est ainsi exclusivement au vu de la

88. Le tribunal indique se référer aux " rules of customary international law as they have evolved" (CIRDI, Philip Morris Brands SARL e.a. c. Uruguay, $\mathrm{n}^{\circ} \mathrm{ARB} / 10 / 7$, sentence du 8 juillet 2016, § 290). Sur la doctrine des police powers envisagée en lien avec le droit de la responsabilité, voir infra, II, A, 5.

89. Ibid., $\S \S 292$ et s. Citation au $§ 295$, avec les citations des décisions arbitrales aux $\$ \S 296$ et s.

90. Ibid., § 301 .

91. Voir aussi CIRDI, Urbaser SA et Consorcio de Aguas Bilbao Bizkaia, Bilbao Biskaia Ur Partzuergoa c. Argentine, $\mathrm{n}^{\circ} \mathrm{ARB} / 07 / 26$, sentence, 8 décembre 2016, $\S \S 1195$ et $\mathrm{s}$. où le tribunal, pour établir que les sociétés, en tant que sujets du droit international, sont liées par des obligations de droit international, s'appuie successivement sur les " Principes directeurs relatifs aux entreprises et aux droits de l'homme: mise en œuvre du cadre de référence 'protéger, respecter et réparer' des Nations Unies " ( Principes Ruggie »), la Déclaration universelle des droits de l'homme de 1948, l’observation générale du Comité pour les droits économiques, sociaux et culturels $n^{\circ} 15$ de 2002 sur le droit à l'eau, la résolution sur cette même question de l'Assemblée générale de l'ONU 64/292 du 28 juillet 2010, la déclaration tripartite de l'OIT de 1977 sur les entreprises multinationales et la politique sociale. Sur cette question, voir infra, III, B, 2 (Droit applicable) et III, C, 1 (Demandes reconventionnelles).

92. Voir le Troisième rapport sur la responsabilité des États du rapporteur spécial James Crawford, A/CN.4/507/Add.1 (2000), § 211.

93. CIRDI, Saint-Gobain Performance Plastics Europe c. Venezuela, n ARB/12/13, décision sur la responsabilité et les principes de la réparation, 30 décembre $2016, \S 890$. À noter que la sentence Autopista s'appuie elle-même sur des " précédents » jurisprudentiels pour établir l'absence de caractère coutumier des intérêts composés (CIRDI, Autopista Concesionada de Venezuela, CA c. Venezuela, $\mathrm{n}^{\circ} \mathrm{ARB} / 00 / 5$, sentence, 23 septembre 2003, §395).

94. CIRDI, Saint-Gobain Performance Plastics Europe c. Venezuela, § 891. 
pratique arbitrale que le tribunal estime que la règle est devenue celle des intérêts composés, sauf circonstances particulières que le défendeur doit établir. Au demeurant, le tribunal ne précise pas que la règle qu'il constate demeure une lex specialis propre au droit des investissements.

La méthode du tribunal de l'affaire Windstream Energy pour déterminer l'état du droit coutumier auquel renvoie l'article 1105, $\S 1^{\text {er }}$, de l'ALENA ${ }^{95}$ n'est guère différente. Le tribunal commence par noter avec orthodoxie que « in principle the content of a rule of customary international law such as the minimum standard of treatment can best be determined on the basis of evidence of actual State practice establishing custom that also shows that the States have accepted such practice as law (opinio juris) »96. Mais en l'absence de tels éléments fournis par les parties, le tribunal indique devoir se contenter d'autres preuves de caractère indirect ( other, indirect evidence "), telles que "decisions taken by other NAFTA tribunals that specifically address the issue of interpretation and application of Article 1105(1) of NAFTA, as well as relevant legal scholarship " ${ }^{97}$. Ce recours à la jurisprudence d'investissement et à la doctrine (elle-même nourrie à la jurisprudence) pour déterminer le droit coutumier est justifié de différentes manières par le tribunal.

Tout d'abord le «Tribunal cannot simply declare non liquet " 98 . À première vue, l'argument n'est en l'espèce pas dénué de tout bon sens pratique dans la mesure où les arbitres doivent appliquer le droit coutumier - c'est ce que leur intime l'article 1105 de l'ALENA - sans avoir à disposition d'autres éléments que la jurisprudence et la doctrine fournies par les parties. Partant, le seul moyen de remplir leur mission juridictionnelle serait de s'appuyer sur ce corpus restreint ${ }^{99}$. Reste que iura novit arbiter, comme la même sentence l'a admis, et rien n'interdirait aux arbitres de partir par leurs propres moyens à la recherche de la pratique et de l'opinio juris des États en matière de standard minimum de traitement.

Un autre argument avancé par le tribunal est que l'article $38 \mathrm{du}$ Statut de la CIJ mentionne la jurisprudence et la doctrine comme des « moyens auxiliaires de détermination des règles de droit " ${ }^{100}$. La CDI a elle-même reconnu qu'à ce titre les décisions juridictionnelles avaient une importance pratique et la doctrine un rôle indicatif dans la détermination du droit coutumier. Mais elle a en même temps insisté sur le caractère auxiliaire de ces moyens ${ }^{101}$. Ils ne suffisent pas à déterminer le droit coutumier, ils viennent en renfort à d'autres moyens, sans s'y substituer tout bonnement ${ }^{102}$.

95. La sentence Mesa Power cite in extenso la décision de la Commission de libre-échange de l'ALENA selon laquelle "the minimum standard of treatment is an umbrella concept reflecting a set of rules that, over time, has crystallized into customary international law in specific contexts " tant et si bien que la disposition "reflects a standard that develops from State practice and opinio juris, rather than an autonomous, treaty-based standard»(CPA (CNUDCI), Mesa Power Group, LLC c. Canada, ${ }^{\circ}$ 2012-17, sentence, 24 mars 2016, § 491).

96. CPA, Windstream Energy LLC c. Canada, n²013-22, sentence, 27 septembre 2016, § 351.

97. Ibid.

98. Ibid.

99. Ibid., § 352 .

100. Ibid., note 742 .

101. Projets de conclusion sur la détermination du droit international coutumier, conclusion 13 (Décisions de juridiction) et conclusion 14 (Doctrine) et leurs commentaires. On notera que l'intérêt représenté par la doctrine pour la CDI ne concerne pas son analyse de la jurisprudence mais la « collecte des informations sur la pratique des États ", l'analyse qu'elle en fait, les divergences dans cette pratique qu'elle relève, l'absence ou la formation éventuelles de règles qu'elle en déduit, et l'évaluation qu'elle fait du droit ( $\$ 2$ du commentaire de la Conclusion 14).

102. Voir les conclusions 6 (Formes de la pratique), 10 (Formes de preuve de l'acceptation comme étant le droit (opinio juris)), 11 (Traités), 12 (Résolutions d'organisations internationales et de conférences intergouvernementales). 
Ultime argument, plus fragile encore : de nombreux autres tribunaux ont eux aussi déterminé le droit coutumier à partir de la jurisprudence et la doctrine sans s'attacher à la pratique des États, au premier rang desquels la commission mixte de l'affaire Neer dont la décision est présentée en jurisprudence comme reflétant le droit coutumier - celui de 1926 du moins ${ }^{103}$. Il est vrai que cette décision, qui tient à peine sur trois pages dans le Recueil des sentences arbitrales des Nations Unies ${ }^{104}$, règle en un court paragraphe la question du standard de traitement des étrangers, dont le contenu est tiré de brèves observations sur le déni de justice par la doctrine de l'époque (Moore, de Lapradelle et Politis), sans que la pratique et l'opinio juris des États soient même évoqués. Qu'il se fût agi à l'époque d'une règle tirée de la pratique générale des États et acceptée par eux comme étant le droit, ou, plus certainement, d'un principe importé des droits des " nations civilisées ", voire une manifestation d'impérialisme juridique dénuée de fondement dans le droit positif, peu importe. La règle a depuis acquis une valeur coutumière dont la preuve aurait pu sans grand peine être établie par le tribunal de l'affaire Windstream Energy au vu de la pratique des États - pas de non liquet donc.

L'exemple de la sentence Mesa Power pourrait encore être cité, dans laquelle le tribunal fait état de deux courants jurisprudentiels contradictoires relatifs au contenu du standard minimum coutumier, avant d'en choisir un sans aucune espèce de justification ${ }^{105}$. Le droit coutumier est ainsi déterminé par l'« opinion » de trois arbitres (voire de deux si un membre du tribunal est dissident), dont les conclusions seront par la suite susceptibles de contribuer à l'identification de la norme coutumière par d'autres tribunaux.

Point n'est besoin de multiplier les exemples pour observer ce phénomène autopoiétique de fixation de règles coutumières par le précédent arbitral, qui revient à brouiller la distinction entre les sources coutumière et jurisprudentielle ${ }^{106}$. Dans la plupart des cas, ce sont finalement la pratique (même pas nécessairement) généralisée des tribunaux arbitraux d'investissement et l'opinio juris (variable) que leurs décisions expriment - mâtinées d'écrits doctrinaux eux-mêmes hétérogènes - qui suffisent selon les arbitres à déterminer le contenu des règles coutumières, sans que d'autres moyens de détermination du droit international coutumier soient mobilisés. Il n'est pas certain que les travaux de la CDI en cours suffisent à remettre de l'ordre dans la pratique arbitrale déficiente.

\section{Principes généraux}

Il est assez courant que les tribunaux arbitraux d'investissement convoquent dans leurs décisions des principes généraux dont la teneur ne prête pas nécessairement à discussion mais dont la source (coutumière, jurisprudentielle, principes généraux de droit au sens de l'article 38 du Statut de la CIJ, autre...) demeure fort

103. Voir CPA (CNUDCI), Mesa Power Group, LLC c. Canada, $\mathrm{n}^{\circ} 2012-17$, sentence, 24 mars 2016 , $\S \$ 496$ et s. ; CIRDI, Crystallex International Corporation c. Venezuela, ${ }^{\circ} \mathrm{ARB}(\mathrm{AF}) / 11 / 2$, sentence, 4 avril $2016, \S 534$.

104. Vol. IV, pp. 60-62.

105. CPA (CNUDCI), Mesa Power Group, LLC c. Canada, § 501 ("Having considered the Parties' positions and the authorities cited by them, the Tribunal is of the opinion that the decision in Waste Management II correctly identifies the content of the customary international law minimum standard of treatment found in Article $1105 »)$.

106. Voir aussi Ch. comm. Stockholm, Charanne BV et Construction Investment SARL c. Espagne, $\mathrm{n}^{\circ}$ 062/2012, sentence, 21 janvier 2016, $\$ 486$; CPA (CNUDCI), Murphy Exploration \& Production Company - International c. Équateur, sentence finale partielle, 6 mai 2016, § 482 ; CIRDI, Corona Materials LLC c. République dominicaine, $\mathrm{n}^{\circ} \mathrm{ARB}(\mathrm{AF}) / 14 / 3$, sentence du 31 mai 2016, $\S 254$ et s. 
imprécise ${ }^{107}$. Il faut dire que l'usage du terme «principe» semble parfois constituer un expédient permettant aux tribunaux de se dispenser de procéder à la démonstration de l'existence de la norme mise en œuvre. Cela ne pose guère de problème lorsque le «principe » invoqué est celui de l'effet utile ${ }^{108}$, de l'économie procédurale ${ }^{109}$, pacta sunt servanda ${ }^{110}$, nemo auditur ${ }^{111}$, de l'estoppel ${ }^{112}$, ou désigne le dictum sur la réparation de la CPJI dans l'affaire de l'Usine de Chorzów ${ }^{113}$; cela est plus gênant quand des " principes » sont tirés à la va-vite de décisions arbitrales qui ne font pas nécessairement l'unanimité ${ }^{114}$.

L'intérêt des décisions arbitrales en matière de principes généraux tient moins à la mise en œuvre - à divers titres : directement normatif ${ }^{115}$, interprétatif ${ }^{116}$ - de règles déjà bien établies qu'à la consécration ou à l'inverse au rejet de nouveaux principes. Les parties en litige ne sont en effet pas les dernières à se prévaloir de normes dont elles cherchent à masquer le caractère contesté ou non généralisé en les parant de la qualification de "principe général ». Il revient alors au tribunal de séparer le bon principe de l'ivraie, en remontant de manière plus ou moins détaillée et argumentée à sa source, ce qui ne doit pas occulter le pouvoir bien commode qu'ont les arbitres de sélectionner les principes selon l'orientation qu'ils entendent donner à leur décision.

Le tribunal de l'affaire Lundin Tunisia a ainsi observé que le mécanisme «fork in the road ", en vertu duquel un investisseur doit opérer un choix définitif et irrévocable entre le recours au juge interne ou au juge arbitral ${ }^{117}$, «ne correspond pas à un principe général de droit. Il ne s'applique qu'en présence d'un texte le prévoyant ${ }^{118}$. En l'occurrence, c'est par la généralisation coutumière des clauses "fork in the road" que le mécanisme semblerait pouvoir accéder au rang de principe. Le tribunal de l'affaire Crystallex laisse pour sa part entendre que la protection des attentes légitimes en tant qu'élément du traitement juste et équitable est en passe d'acquérir ce statut, au terme d'une évolution assez classique : sans opérer

107. Voir par exemple au sujet de la doctrine de l'abus de droit, CNUDCI, The Renco Group Inc. $c$. Pérou, UNCT/13/1, sentence partielle sur la compétence, 15 juillet 2016, $\S 175$ et s. Contra voir CIRDI, Tenaris SA et Talta - Trading e Marketing Sociedade Unipessoal Lda. c. Venezuela, $\mathrm{n}^{\circ} \mathrm{ARB} / 12 / 23$, sentence, 12 novembre 2016, $\S 181,192$, où la référence aux principes généraux de droit reconnus par les nations civilisées est explicite (au sujet du siège effectif des sociétés).

108. CIRDI, Joseph Houben c. Burundi, n ARB/13/7, sentence, 12 janvier 2016, § 156. Voir supra, A, 1 (Interprétation des traités).

109. CIRDI, Vestey Group Limited c. Venezuela, ${ }^{\circ}$ ARB/06/4, sentence, 15 avril 2016, § 318.

110. CIRDI, RREEF Infrastructure (GP) Limited et RREEF Pan-European Infrastructure Two Lux SARL c. Espagne, n ${ }^{\circ} \mathrm{ARB} / 13 / 30$, décision sur la compétence, 6 juin $2016, \S 85$.

111. CIRDI, Tenaris SA et Talta - Trading e Marketing Sociedade Unipessoal Lda. c. Venezuela, $\mathrm{n}^{\circ}$ ARB/12/23, sentence, 12 novembre 2016, § 362.

112. CIRDI, Vestey Group Limited c. Venezuela, $\mathrm{n}^{\circ}$ ARB/06/4, sentence, 15 avril 2016, § 118, § 257 ;

CIRDI, Getma International, NCT Necotrans, Getma International Investments, NCT Infrastructure \& Logistique c. Guinée, n ARB/11/29, sentence, 16 août 2016, § 343.

113. CPA (CNUDCI), Copper Mesa Mining Corporation c. Équateur, n 201-2, sentence, 15 mars $2016, \S \S 7.29,8.10$ et $\mathrm{s}$.

114. CIRDI, Ampal-American Israel Corp. et al. c. Égypte, $\mathrm{n}^{\circ} \mathrm{ARB} / 12 / 11$, décision sur la compétence, $1^{\text {er }}$ février 2016 , $\S 301$, où le tribunal s'appuie un peu rapidement sur un principe qu'il estime bien établi de droit international selon lequel un tribunal constitué sur le fondement d'un traité d'investissement n'a pas compétence concernant les investissements réalisés en violation du droit national. Voir aussi Comité ad hoc CIRDI, TECO Guatemala Holdings LLC c. Guatemala, ${ }^{\circ}$ ARB/10/23, décision sur l'annulation, 5 avril 2016, § 375 au sujet du principe « costs follow the event ».

115. Ex. : CPA (CNUDCI), Copper Mesa Mining Corporation c. Équateur, ${ }^{\circ}$ 201-2, sentence, 15 mars $2016, \S \S 7.29,8.10$ et $\mathrm{s}$.

116. Ex. : CNUDCI, The Renco Group Inc. c. Pérou, UNCT/13/1, sentence partielle sur la compétence, 15 juillet 2016, $§ 154$ (principe de bonne administration de la justice) ; CIRDI, Joseph Houben c. Burundi, $\mathrm{n}^{\circ} \mathrm{ARB} / 13 / 7$, sentence, 12 janvier 2016, § 156 (principe d'effet utile).

117. Voir Arnaud DE NANTEUIL, Droit international de l'investissement, Paris, Pedone, 2017, $2^{\mathrm{e}}$ éd., pp. 149 et $\mathrm{s}$.

118. CIRDI, Lundin Tunisia BV c. Tunisie, $\mathrm{n}^{\circ}$ ARB/12/30, sentence, 22 décembre 2015, § 178. 
de démonstration mais s'appuyant sur une sentence ayant abordé la question, le tribunal rappelle que la doctrine des attentes légitimes " has its origins in principles of domestic administrative law in various legal systems, and finds increasing recognition both in civil and common law countries ", y compris au sein du droit de l'État défendeur. Le tribunal note aussi que plusieurs tribunaux arbitraux ont conclu que cette doctrine "is firmly rooted in arbitral practice "; et le tribunal de l'espèce "agrees with the majority of investment tribunals which have concluded that protection of legitimate expectations is now considered part of the FET standard "119. La norme de protection des attentes légitimes résulte ainsi de l'imbrication de diverses sources parmi lesquelles la jurisprudence semble finalement la plus déterminante.

Le pouvoir qu'ont les tribunaux de dégager des principes par accrétion à partir de la jurisprudence internationale et arbitrale ${ }^{120}$ ne sera pas employé s'il débouche sur une solution non souhaitée par les arbitres. Ainsi le tribunal de l'affaire Renco a-t-il observé que les tribunaux internationaux (CIJ, CEDH) avaient fait usage d'un principe de «divisibilité » dans la mise en œuvre des réserves des États aux traités ou des déclarations de juridiction obligatoire. Le tribunal a situé sur le même plan le principe d'autonomie de la clause compromissoire, par lequel les tribunaux arbitraux isolent sans peine la convention d'arbitrage du reste du contrat ${ }^{121}$. Néanmoins les arbitres ont semblé exclure que la divisibilité puisse constituer « a general principle of international law » ${ }^{122}$, au motif qu'elle n'a jamais été mise œuvre par les tribunaux d'investissement au sujet de la renonciation de l'investisseur aux voies de recours internes, et qu'au contraire l'offre d'arbitrage des États contenue dans les traités d'investissement ne tolère pas que les investisseurs insèrent des réserves, divisibles ou pas, dans leur acception de l'offre d'arbitrage des États ${ }^{123}$. Mais en l'occurrence les arbitres semblent moins rejeter le principe même de divisibilité, d'ailleurs bien connu des différents droits nationaux des obligations et décliné dans la jurisprudence citée par la sentence, que rejeter son applicabilité à la réserve incluse dans la déclaration de renonciation en raison de la teneur de la clause de règlement des différends applicable.

Sans assumer pleinement la méthodologie aboutissant à consacrer des principes généraux de droit au sens de l'article 38 du Statut de la CIJ, il n'est pas rare que les arbitres s'appuient sur les droits internes pour dégager des règles applicables dans l'arbitrage d'investissement. Ce peut être une affirmation péremptoire fondée sur l'idée que se font les arbitres de l'État de droit ${ }^{124}$. Ce peut n'être aussi qu'une brève incise, par exemple pour appuyer la conclusion selon laquelle les parties doivent révéler au tribunal des informations connexes de nature à exercer une influence décisive sur la solution ${ }^{125}$. Inversement, un lourd travail de droit

119. CIRDI, Crystallex International Corporation c. Venezuela, $\mathrm{n}^{\circ} \mathrm{ARB}(\mathrm{AF}) / 11 / 2$, sentence, 4 avril $2016, \S 546$.

120. Au sujet du principe «iura novit curia » renommé «iura novit arbiter », voir CIRDI, Vestey Group Limited c. Venezuela, $\mathrm{n}^{\circ} \mathrm{ARB} / 06 / 4$, sentence, 15 avril 2016, $\S 118$ (le tribunal cite la jurisprudence arbitrale d'investissement ainsi que l'arrêt de la CIJ dans l'affaire Compétence en matière de pêcheries (République fédérale d'Allemagne c. Islande)).

121. CNUDCI, The Renco Group Inc. c. Pérou, UNCT/13/1, sentence partielle sur la compétence, 15 juillet $2016, \S 170$.

122. Ibid., § 172 .

123. Voir supra, A, 2.

124. CIRDI, Getma International, NCT Necotrans, Getma International Investments, NCT Infrastructure \& Logistique c. Guinée, $\mathrm{n}^{\circ} \mathrm{ARB} / 11 / 29$, sentence, 16 août $2016, \S 386$ : « toute mesure administrative ou acte de puissance publique doit être dûment justifié et proportionnel au but qu'il poursuit ; cela constitue un principe général qui s'applique aussi en droit guinéen. Ce principe est essentiel dans tout État de droit afin d'éviter l'arbitraire".

125. CIRDI, Standard Chartered Bank (Hong Kong) Ltd c. Tanzania Electric Supply Company Ltd, $\mathrm{n}^{\circ} \mathrm{ARB} / 10 / 20$, sentence, 12 septembre 2016, $\S 333$, où le tribunal indique en note de bas de page : «[i]n 
comparé dans une perspective historique peut être conduit, à l'instar de la démarche suivie par le tribunal de l'affaire Pac Rim, qui a tiré le droit de regard qu'ont les propriétaires du sol sur l'utilisation du sous-sol d'une longue analyse ayant pour point de départ la vieille maxime latine cuius est solum, eius est usque ad coelum et ad inferos dont la mise en œuvre dans plusieurs systèmes nationaux a été retracée avec force références ${ }^{126}$. En l'occurrence le tribunal n'a pas cherché à consacrer une norme internationale mais à « conforter " son interprétation du droit salvadorien ${ }^{127}$. La règle dégagée n'est pas présentée comme un principe général de droit et, au reste, elle n'en remplit pas les fonctions.

La philosophie des principes généraux de droit repose sur l'absence de prévalence d'un droit national ou d'une famille juridique sur les autres, ce qui est le meilleur bouclier à l'impérialisme juridique même si les deux familles de droit que sont la Common Law et le droit romano-germanique, largement diffusées via la colonisation, sont clairement dominantes. À cet égard, on relèvera avec attention l'incise du tribunal Crystallex sur les standards de preuve :

"The Parties have debated whether the Tribunal should apply a "balance of probabilities" or a "sufficient degree of certainty" test. In the Tribunal's view, these tests chiefly reflect common law concepts (whereby the balance of probabilities standard, or preponderance of the evidence, is opposed to the "beyond a reasonable doubt" standard, normally used in criminal matters). In continental legal systems, by contrast, the matter of proof is left to the personal appreciation of the judge (inner conviction, "intime conviction"). If the judge is persuaded of the truth of a certain matter, then the standard of proof has been met. »128

Rejetant ces deux approches au motif qu'il constitue un organe arbitral international établi dans le cadre d'un traité, le tribunal a déterminé une série de « principes » relatifs à la preuve qu'il entendait suivre. À l'heure où les concepts de Common Law prospèrent plus que jamais dans le contentieux international - pour diverses raisons dont la montée en puissance dans ce contentieux des grandes law firms anglo-américaines n'est pas la dernière -, ce rappel à l'ordre est salutaire. Mais on relèvera que la plupart des principes posés sont repris de décisions arbitrales antérieures, ce qui témoigne de la part déterminante de la source jurisprudentielle dans le contentieux d'investissement. En ce domaine, les approches antagonistes en matière de preuve des systèmes romano-germanique et de Common Law semblaient exclure la formulation par comparaison de principes généraux. Cette décision est d'ailleurs à rapprocher de la sentence Lundin Tunisia. Sans se référer aux «principes généraux de droit » le tribunal en a néanmoins mis en œuvre la méthode pour rejeter la demande de dommages-intérêts punitifs faite par l'État défendeur à titre reconventionnel : "Si dans certains systèmes juridiques les dommages-intérêts punitifs peuvent être accordés par les tribunaux en plus des dommages-intérêts compensatoires, la plupart des systèmes juridiques issus du droit continental ne les admettent pas ${ }^{129}$. La comparaison rapidement non concluante des règles de réparation issues des deux principales familles de droit

most judicial systems rules of professional conduct would place counsel under an obligation to the court to make such disclosure».

126. CIRDI, Pac Rim Cayman LLC c. Salvador, n ARB/09/12, sentence, 14 octobre 2016 , $\S 8.59$ et s. 127. Ibid., $\$ 8.54$ : le tribunal " has based its decision on the issue of legal interpretation under Salvadoran law. However, the Tribunal has taken comfort from other legal materials lending support to that decision".

128. CIRDI, Crystallex International Corporation c. Venezuela, $n^{\circ} \mathrm{ARB}(\mathrm{AF}) / 11 / 2$, sentence, 4 avril $2016, \S 865$.

129. CIRDI, Lundin Tunisia BV c. Tunisie, $\mathrm{n}^{\circ}$ ARB/12/30, sentence, 22 décembre 2015, § 381 . 
exclut ainsi que la règle propre à seulement l'une d'entre elles accède au rang de principe international.

L'échec de la méthode des principes généraux de droit et le rejet des prétentions reconventionnelles de l'État défendeur dans l'affaire Lundin Tunisia sont à relativiser si l'on prend en considération les vastes champs que l'affaire Urbaser invite à défricher ${ }^{130}$. En reconnaissant, en effet, que les investisseurs sont des sujets de droit international, astreints à des obligations internationales en matière de droits de l'homme, dont un tribunal arbitral, sur le fondement d'une demande reconventionnelle de l'État, pourrait sanctionner l'irrespect en condamnant l'investisseur à indemniser les dommages qu'il a causés, les arbitres ont laissé une question, pourtant fondamentale, irrésolue : quel serait le régime de responsabilité applicable à l'investisseur ? Dans la logique de la sentence, c'est une responsabilité internationale civile qui devrait être mise en œuvre. Or, en matière de responsabilité des personnes privées, le droit international contemporain ne connaît que de la responsabilité pénale de l'individu. C'est aux arbitres qu'il reviendrait donc d'élaborer un corpus même minimaliste de règles secondaires. Plusieurs options s'offriraient alors à eux. La première reviendrait à appliquer mutatis mutandis le régime de la responsabilité pour fait internationalement illicite de l'État ou des organisations internationales (dont on sait qu'elle "n'est ni civile, ni pénale, mais internationale » ${ }^{131}$ ) au cas des personnes privées. La seconde, qu'on veut croire plus adaptée, consisterait à dégager, à partir de la comparaison des différents mécanismes de responsabilité civile à l'œuvre dans la plupart des systèmes juridiques, des principes communs aptes à être transposés dans l'ordre juridique international sous la forme de principes généraux de droit. On ne saurait mieux mettre en évidence, si besoin était, le potentiel extraordinaire de développement du droit international que représente l'arbitrage d’investissement.

\section{Sources informelles - soft law, doctrine}

La soft law occupe dans le contentieux d'investissement une place qui n'est pas nécessairement inédite devant d'autres juridictions internationales ${ }^{132}$, mais qui surprend tout de même par son ampleur ${ }^{133}$. Il est ainsi courant que les tribunaux arbitraux se réfèrent dans leur motivation à des résolutions, directives, working papers, rapports, et autres projets, provenant d'organisations publiques comme privées ${ }^{134}$ - les règles de l'International Bar Association (IBA) faisant l'objet d'une attention récurrente en matière procédurale ${ }^{135}$. La doctrine, de son côté, bénéficie dans le contentieux transnational d'une mobilisation inespérée pour les «publicistes [et les privatistes en l'occurrence] les plus qualifiés » dont l'audience devant

130. CIRDI, Urbaser SA et Consorcio de Aguas Bilbao Bizkaia, Bilbao Biskaia Ur Partzuergoa $c$. Argentine, $\mathrm{n}^{\circ} \mathrm{ARB} / 07 / 26$, sentence, 8 décembre 2016. Voir infra, III, C, 1.

131. Rapport de la CDI sur les travaux de sa 50e session, Ann. CDI, 1998, vol. II, A/53/10, p. 74, § 292.

132. Voir Sébastien VAN DRooghenbroeck, "Le soft law et la Cour européenne des droits de l'homme ", in Mihaela A. AILINCAI (dir.), Soft Law et droits fondamentaux, Paris, Pedone, 2017, pp. 185 et s.

133. Voir déjà cette chronique, cet Annuaire, 2015, pp. 863 et s.

134. CIRDI, Crystallex International Corporation c. Venezuela, $n^{\circ} \mathrm{ARB}(\mathrm{AF}) / 11 / 2$, sentence, 4 avril 2016, $\S \S 883-884$ (directives et standards de l'Institut canadien des mines en matière d'évaluation des propriétés minières).

135. S'agissant des règles de l'IBA sur l'administration de la preuve dans l'arbitrage international (2010), voir CIRDI, Churchill Mining PLC et Planet Mining Pty Ltd c. Indonésie, $\mathrm{n}^{\circ}$ ARB/12/14 et 12/40, sentence, 6 décembre 2016, § 249 ; CPA (CNUDCI), South American Silver Ltd (Bermuda) c. Bolivie, $\mathrm{n}^{\circ} 2013-15$, ordonnance procédurale $\mathrm{n}^{\circ} 14,1^{\mathrm{er}}$ avril $2016, \S 26$ et ordonnance procédurale $\mathrm{n}^{\circ} 15,9$ avril $2016, \S 21$. 
d'autres juridictions internationales ou nationales reste à peu près nulle - à la lecture, du moins, de la motivation de leurs décisions ${ }^{136}$.

Certes, formellement on ne saurait assimiler la recommandation d'une organisation internationale et la monographie d'un professeur de droit. Mais les frontières en la matière ne sont pas étanches. Le rapport final du groupe d'étude de la Commission du droit international des Nations Unies sur la clause de la nation la plus favorisée (2015), auquel une sentence de 2016 se réfère ${ }^{137}$, constitue ainsi, d'un point de vue strictement formel, un acte d'organisation internationale ; substantiellement, il peut néanmoins être rattaché à la doctrine ${ }^{138}$. Surtout, dans l'arbitrage d'investissement la doctrine remplit des fonctions analogues à celles des normes tirées de sources informelles auxquelles elle peut dès lors être assimilée. Ainsi, plus que le recensement des divers instruments soft ou des références doctrinales employées, c'est la fonction commune assignée à ces sources informelles qui mérite la plus grande attention.

La part étonnante de ces dernières dans la déduction arbitrale de l'existence ou du contenu de normes coutumières a déjà été relevée ${ }^{139}$. Dans d'autres cas de figure, la norme soft ou doctrinale ${ }^{140}$ est mobilisée par les arbitres en ce qu'elle fait état du droit "dur» 141 , ou renseigne à tout le moins sur l'état du droit positif ${ }^{142}$. Le cas est très parlant des comités $a d$ hoc du CIRDI qui se réfèrent directement dans leurs décisions à la « Note d'information » («Background Paper») sur l'annulation réalisée par le Secrétariat du CIRDI ${ }^{143}$. Ce document retrace l'historique de l'article 52 de la convention de Washington (travaux préparatoires), rappelle en détail (règlement d'arbitrage à l'appui) les éléments de la procédure d'annulation, et surtout compile la jurisprudence pertinente des comités ad hoc du CIRDI en

136. Voir Franck LATTY, "Doctrine : eut du succès au temps du cinéma muet ", in Hervé AsCENSIO et al. (dir.), Dictionnaire des idées reçues en droit international, Paris, Pedone, 2017, pp. 161-167.

137. CPA (CNUDCI), Venezuela US, SRL c. Venezuela, $\mathrm{n}^{\circ} 2013-34$, sentence partielle sur la compétence, 26 juillet $2016, \S 102$.

138. Voir CIRDI, RREEF Infrastructure (GP) Limited et RREEF Pan-European Infrastructure Two Lux SARL c. Espagne, $\mathrm{n}^{\circ} \mathrm{ARB} / 13 / 30$, décision sur la compétence, 6 juin 2016, $\S 82$, où une référence est faite aux travaux de la CDI sur la fragmentation du droit international.

139. Voir supra, B (Coutume).

140. Comité ad hoc CIRDI, EDF SA et SAUR International SA c. Argentine, ${ }^{\circ} \mathrm{ARB} / 03 / 23$, décision sur l'annulation, 5 février 2016, $\$ \$ 330,335$ (références explicites à J. Crawford, State Responsibility: The General Part, 2013).

141. Voir par ex. CIRDI, Saint-Gobain Performance Plastics Europe c. Venezuela, n ARB/12/13, décision sur la responsabilité et les principes de la réparation, 30 décembre 2016, §627 (référence aux Directives de la Banque mondiale sur le traitement des investissements (1992) en tant que reflet, avec l'art. 35 des Articles de la CDI de 2001, du " compensation standard under customary international law ", s'agissant de la fair market value). Dans un sens voisin, voir Ch. comm. Stockholm, Charanne BV et Construction Investment SARL c. Espagne, $n^{\circ}$ 062/2012, sentence, 21 janvier 2016, § 464 (référence au Rapport de 2012 de la CNUCED sur l'expropriation ; voir aussi $\$$ 488-489 où les demandeurs cherchent à se prévaloir de l'étude de la CNUCED sur le traitement juste et équitable, dont le tribunal cite un extrait - un autre tribunal s'appuie d'ailleurs sur cette étude : CIRDI, Garanti Koza LLP c. Turkménistan, $\mathrm{n}^{\circ} \mathrm{ARB} / 11 / 20$, sentence, 19 décembre 2016, § 380); Comité ad hoc CIRDI, Adem Dogan c. Turkménistan, $\mathrm{n}^{\circ} \mathrm{ARB} / 09 / 9$, décision sur l'annulation, 15 janvier 2016, $\$ 118$ (référence à l'ouvrage de Sir Antony AUST, Modern Treaty Law and Practice, au sujet du principe ejusdem generis).

142. Voir CPA (CNUDCI), Copper Mesa Mining Corporation c. Équateur, ${ }^{\circ}$ 201-2, sentence, 15 mars 2016, § 6.60 où référence est faite au Third Restatement on Foreign Relations Law (1986) de l'American Law Institute en matière d'expropriation (6.60); CIRDI, RREEF Infrastructure (GP) Limited et RREEFF Pan-European Infrastructure Two Lux SARL c. Espagne, $\mathrm{n}^{\circ} \mathrm{ARB} / 13 / 30$, décision sur la compétence, 6 juin 2016, § 122 (référence à D. Müller, La protection de l'actionnaire en droit international, 2015 pour faire état des incertitudes de la jurisprudence).

143. Comité ad hoc CIRDI, Adem Dogan c. Turkménistan, $\mathrm{n}^{\circ} \mathrm{ARB} / 09 / 9$, décision sur l'annulation, 15 janvier 2016, § 104 ; Comité ad hoc CIRDI, EDF SA et SAUR International SA c. Argentine, $\mathrm{n}^{\circ} \mathrm{ARB} / 03 / 23$, décision sur l'annulation, 5 février 2016, $\S \$ 167,181$; Comité ad hoc CIRDI, Antoine Abou Lahoud et al. c. $R D C, \mathrm{n}^{\circ} \mathrm{ARB} / 10 / 4$, décision sur l'annulation, 29 mars 2016, § 118. 
la matière ${ }^{144}$. Le comité ad hoc de l'affaire Total n'a pas hésité à citer in extenso plusieurs passages du Background Paper afin de faire état de l'étendue limitée de la procédure d'annulation ${ }^{145}$, ou de préciser le sens de certains motifs d'annulation prévus à l'article 52 de la convention de Washington : excès manifeste de pouvoir ${ }^{146}$, défaut de motifs ${ }^{147}$ ou inobservation grave d'une règle de procédure fondamentale ${ }^{148}$. Le document fournit en somme une synthèse sur laquelle les comités ad hoc s'appuient en toute confiance en tant que reflet du droit positif de l'annulation, ce qui témoigne d'un certain pouvoir normatif du Secrétariat du CIRDI, dont l'interprétation du droit positif (car synthétiser ou compiler c'est aussi interpréter) pourra trouver écho. Mais en matière d'annulation ${ }^{149}$, comme plus généralement pour tout ce qui touche à la convention de Washington ${ }^{150}$, il est une somme doctrinale qui fait tout autant, sinon davantage, autorité auprès des tribunaux arbitraux ou des comités ad hoc: le commentaire de la convention de Washington par Ch. Schreuer - qualifié de «leading commentator » 151 , au point que ses co-auteurs depuis la deuxième édition, L. Malintoppi, A. Reinisch et A. Sinclair, sont fréquemment oubliés dans les citations ${ }^{152}$.

Les normes souples ou doctrinales peuvent aussi jouer un rôle dans l'interprétation des normes conventionnelles - ainsi de la loi type de la CNUDCI sur la passation des marchés publics, utilisée pour interpréter la notion d' "achat » dans l'ALENA ${ }^{153}$, du rapport de la CDI sur la clause de la nation la plus favorisée pour interpréter la clause en question du traité Barbade/Venezuela ${ }^{154}$, des directives de l'IBA relatives aux conflits d'intérêt dans l'arbitrage international en matière de récusation des arbitres ${ }^{155}$, et des nombreux travaux doctrinaux sur le

144. Le Background Paper de 2012 a fait l'objet en 2016 d'une actualisation, intégrant les suites des 37 procédures d'annulation et les 22 nouvelles décisions relatives à l'annulation rendues pendant cette période. Le document est accessible sur le site internet du CIRDI.

145. Comité ad hoc CIRDI, Total SA c. Argentine, $\mathrm{n}^{\circ} \mathrm{ARB} / 04 / 01$, décision sur l'annulation, $1^{\mathrm{er}}$ février 2016, §§ 159-160.

146. Ibid., $\S \S 177-178, \S 183, \S \S 240-241$.

147. Ibid., \$266.

148. Ibid., § 311. Voir aussi Comité ad hoc CIRDI, EDF SA et SAUR International SA c. Argentine, $\mathrm{n}^{\circ} \mathrm{ARB} / 03 / 23$, décision sur l'annulation, 5 février 2016, $§ 181$.

149. Voir par ex. Comité ad hoc CIRDI, Antoine Abou Lahoud et al. c. RDC, $\mathrm{n}^{\circ} \mathrm{ARB} / 10 / 4$, décision sur l'annulation, 29 mars 2016, $\S \S 118,119,130,135$; Comité ad hoc CIRDI, EDF SA et SAUR International $S A$ c. Argentine, $\mathrm{n}^{\circ} \mathrm{ARB} / 03 / 23$, décision sur l'annulation, 5 février 2016, $\$$ 62, 192, 219 ; Comité ad hoc CIRDI, Ion Micula et al. c. Roumanie, $\mathrm{n}^{\circ} \mathrm{ARB} / 05 / 20$, décision sur l'annulation, 26 février 2016, § 292.

150. CIRDI, Ampal-American Israel Corp. et al. c. Égypte, $\mathrm{n}^{\circ} \mathrm{ARB} / 12 / 11$, décision sur la compétence, $1^{\mathrm{er}}$ février 2016, § 337 ; CIRDI, MNSS BV et Recupero Credito Acciaio NV c. Monténégro, ARB(AF)/12/8, sentence, 4 mai 2016, § 163 ; CIRDI, Rusoro Mining Ltd c. Venezuela, ${ }^{\circ} \mathrm{ARB}(\mathrm{AF}) / 12 / 15$, sentence, 22 août 2016, § 263 ; CIRDI, United Utilities (Talinn) BV et Aktsiaselts Tallinna Vesi c. Estonie, ${ }^{\circ}$ ARB/14/24, décision sur la demande de mesures provisoires, 12 mai 2016, § 83 ; Comité ad hoc CIRDI, Bernhard von Pezold et al. c. Zimbabwe, $\mathrm{n}^{\circ} \mathrm{ARB} / 10 / 15$, décision sur la demande de mesures provisoires du demandeur, 17 mars 2016, § 30 ; CIRDI, Vestey Group Limited c. Venezuela, n ARB/06/4, sentence, 15 avril 2016, $\S 194$; Comité ad hoc CIRDI, Tidewater Investment SRL et Tidewater Caribe, CA c. Venezuela, $\mathrm{n}^{\circ} \mathrm{ARB} / 10 / 5$, décision sur la demande de suspension de l'exécution de la sentence, 29 févier $2016, \S 57$.

151. CIRDI, ConocoPhillips Petrozuata BVet al. c. Venezuela, $\mathrm{n}^{\circ} \mathrm{ARB} / 07 / 30$, décision sur la demande de réexamen de la décision du tribunal du 10 mars 2014, 9 février 2016, § 23. Voir aussi Comité ad hoc CIRDI, EDF SA et SAUR International SA c. Argentine, n ARB/03/23, décision sur l'annulation, 5 février 2016, § 192.

152. D'autres travaux de Ch. Schreuer sont fréquemment cités, voir par ex. CIRDI, Garanti Koza LLP c. Turkménistan, $\mathrm{n}^{\circ} \mathrm{ARB} / 11 / 20$, sentence, 19 décembre 2016, § 380 (article de 2005 de l'auteur sur le traitement juste et équitable dans la pratique arbitrale).

153. CPA (CNUDCI), Mesa Power Group, LLC c. Canada, $\mathrm{n}^{\circ}$ 2012-17, sentence, 24 mars 2016, § 414.

154. CPA (CNUDCI), Venezuela US, SRL c. Venezuela, $n^{\circ} 2013-34$, sentence partielle sur la compétence, 26 juillet $2016, \S 102$.

155. CIRDI, Fábrica de Vidrios La Andes, CA et Owens-Illinois de Venezuela, CA c. Venezuela, $\mathrm{n}^{\circ} \mathrm{ARB} / 12 / 21$, décision sur la demande de récusation de L. Yves Fortier, arbitre, 28 mars 2016, § 34 (pertinence des directives de l'IBA en tant que "références utiles " en matière de récusation, même si le tribunal entend se fonder sur la convention de Washington). 
droit international général ${ }^{156}$ ou plus spécifiquement sur l'arbitrage d'investissement ${ }^{157}$, en ce compris le commentaire de Schreuer et al., qui ne se prive pas de fournir des interprétations de la convention de Washington ${ }^{158}$. Un dialogue entre la doctrine et les arbitres est même susceptible de se manifester lorsque le tribunal cite in extenso le commentaire critique d'une de ses décisions antérieures ${ }^{159}$ avant que de le désapprouver assez sèchement ${ }^{160}$. On percevra également l'ironie de la sentence qui apporte la contradiction à un expert intervenu au soutien de l'investisseur en prenant la peine de citer le passage d'un ouvrage dont il est l'auteur ${ }^{161}$. Il faut dire que dans le domaine du droit des investissements plus encore que dans d'autres secteurs du droit international les voies de passage entre la doctrine et la jurisprudence sont nombreuses. Les sociologues du droit ne manqueront pas de constater à cet égard que de nombreux éminents internationalistes exercent des fonctions d'arbitre ${ }^{162}$.

Dans des cas plus rares, enfin, la norme souple peut déterminer une solution que d'autres sources de droit ne permettent pas de fonder ${ }^{163}$. En matière procédurale, en particulier, il n'est pas rare que les tribunaux se réfèrent à des instruments extérieurs, ce que le tribunal de l'affaire United Utilities a tenté de justifier par une interprétation des instruments CIRDI selon laquelle, si les parties au litige ne se mettent pas d'accord sur les règles procédurales applicables à leur différend, « the arbitral tribunal enjoys an equally ample authority to establish the applicable

156. Voir par ex. CIRDI, Ampal-American Israel Corp. et al. c. Égypte, n ARB/12/11, décision sur la compétence, $1^{\text {er }}$ février 2016, $\$ 337, \S 219$ (référence à l'ouvrage de B. Cheng, General Principes of Law as Applied by International Courts and Tribunals, 1953); Ch. comm. Stockholm, Evrobalt LLC c. Moldavie, arbitrage d'urgence EA 2016/082, sentence sur les mesures d'urgence, 30 mai 2016, § 38 (référence à S. Rosenne, The Law and Practice of the International Court, 2006).

157. Voir par ex. CIRDI, Tenaris SA et Talta - Trading e Marketing Sociedade Unipessoal Lda. c. Venezuela, $\mathrm{n}^{\circ} \mathrm{ARB} / 12 / 23$, sentence, 29 janvier 2016, $\S \S 137-138,144,167,291$ (références à l'ouvrage de Z. Douglas, The International Law of Investment Claims, 2009).

158. Voir CIRDI, ConocoPhillips Petrozuata BV et al. c. Venezuela, ${ }^{\circ}$ ARB/07/30, décision sur la demande de réexamen de la décision du tribunal du 10 mars 2014, 9 février 2016, § 23; Comité ad hoc CIRDI, Adem Dogan c. Turkménistan, n ARB/09/9, décision sur l'annulation, 15 janvier 2016, $\S ~ 28$, $103,165$.

159. Charles N. Brower et Paula F. HenIN, «Res Judicata: ConocoPhilips v. Venezuela, ICSID Case $n^{\circ} A R B / 07 / 30$ ", in Meg KINNEAR et al. (ed.), Building International Investment Law : The First 50 years of ICSID, Alphen aan den Rijn, ICSID/Wolters Kluwer, 2016, pp. 67-69.

160. CIRDI, ConocoPhillips Petrozuata BV et al. c. Venezuela, $\mathrm{n}^{\circ} \mathrm{ARB} / 07 / 30$, décision sur la demande de réexamen de la décision du tribunal du 10 mars 2014, 9 février 2016, § 34. L'arbitre dissident mentionne également ce commentaire doctrinal dans son opinion.

161. CIRDI, Philip Morris Brands SARL, Philip Morris Products SA c. Uruguay, n ${ }^{\circ}$ ARB/10/7, sentence, 8 juillet 2016, § 533 (Paulsson, Denial of Justice in International Law, 2005).

162. Pour s'en tenir aux professeurs de droit de nationalité française ayant participé à un tribunal ayant rendu une décision en 2016 : L. Boisson de Chazournes (CIRDI, Garanti Koza LLP c. Turkménistan, $\mathrm{n}^{\circ} \mathrm{ARB} / 11 / 20$, sentence, 19 décembre 2016 ; CIRDI, Crystallex International Corporation c. Venezuela, $\mathrm{n}^{\circ} \mathrm{ARB}(\mathrm{AF}) / 11 / 2$, sentence, 4 avril 2016) ; P.-M. Dupuy (CIRDI, Vestey Group Limited c. Venezuela, $\mathrm{n}^{\circ} \mathrm{ARB} / 06 / 4$, sentence, 15 avril 2016 ; CIRDI, Corona Materials LLC c. République dominicaine, $\mathrm{n}^{\circ} \mathrm{ARB}(\mathrm{AF}) / 14 / 3$, sentence du 31 mai 2016) ; E. Gaillard (CIRDI, MNSS BV et Recupero Credito Acciaio NV c. Monténégro, ARB(AF)/12/8, sentence, 4 mai 2016) ; P. Mayer (CIRDI, Menzies Middle East and Africa SA et Aviation Handling Services International Ltd c. Sénégal, n ARB/15/21, sentence, 5 août 2016) ; A. Pellet (CIRDI, RREEF Infrastructure (GP) Limited et RREEF Pan-European Infrastructure Two Lux SARL c. Espagne, $\mathrm{n}^{\circ} \mathrm{ARB} / 13 / 30$, décision sur la compétence, 6 juin 2016) ; B. Stern (CIRDI, Joseph Houben c. Burundi, $\mathrm{n}^{\circ} \mathrm{ARB} / 13 / 7$, sentence, 12 janvier 2016 ; CIRDI, Tenaris SA et Talta - Trading e Marketing Sociedade Unipessoal Lda. c. Venezuela, $\mathrm{n}^{\circ} \mathrm{ARB} / 12 / 23$, sentence, 12 novembre 2016 ; etc.).

163. Voir CIRDI, Crystallex International Corporation c. Venezuela, $n^{\circ} \mathrm{ARB}(\mathrm{AF}) / 11 / 2$, sentence, 4 avril $2016, \S \S 883-884$, où le tribunal retient qu'en matière de "propriétés en développement ", les directives et standards de l'Institut canadien des mines en matière d'évaluation des propriétés minières, "which are considered as important standards in the industry ", recommandent l'application d'une méthodologie basée sur les revenus et le marché, méthodologie que suivra le tribunal. 
procedure ${ }^{164}$. Sur ce fondement, un tribunal arbitral est libre de prévoir l'application des règles de l'International Bar Association en matière d'administration de la preuve ${ }^{165}$, qui perdent par ce renvoi leur caractère soft. Mais même lorsque l'instrument externe ne bénéficie pas d'une telle onction, il peut être revêtu d'une certaine pertinence. À cet égard, l'affaire Hydro Srl est assez révélatrice de l'usage de la soft law par les arbitres. Était à l'examen du tribunal la condition de la nécessité en matière d'indication de mesures conservatoires. En raison des différentes approches en la matière dans la jurisprudence arbitrale d'investissement, le tribunal a décidé de suivre le «standard ", conforme selon lui aux positions des parties, fixé à l'article 17A de la loi type de la CNUDCI sur l'arbitrage commercial international ${ }^{166}$. Ainsi, plutôt que de procéder à une analyse du critère en mobilisant des sources « tangibles » de droit (on pourrait penser à la jurisprudence de la CIJ, ou aux principes généraux de droit qu'il était envisageable de dégager des lois nationales sur l'arbitrage, que la loi type de la CNUDCI a vocation à inspirer), les arbitres se sont contentés de donner directement effet à des normes soft conçues dans un contexte autre que le droit des investissements. La proximité sociologique des membres du tribunal avec l'arbitrage commercial international n'en est sans doute pas la moins mauvaise explication, mais l'exemple témoigne de l'approche décomplexée des tribunaux à l'égard des sources informelles. Qui plus est, le fait qu'un tribunal se saisisse ainsi d'une norme soft viendra en conforter par la suite l'autorité vis-à-vis d'autres tribunaux. Les arbitres de l'affaire ConocoPhillips ont ainsi repris à leur compte la directive de l'IBA «endorsed by an ICSID decision » selon laquelle la non divulgation d'un élément par un arbitre ne suffit pas à entraîner sa récusation ${ }^{167}$. Force est de constater que la référence dans une sentence à une norme soft ou à une référence doctrinale 168 est susceptible d'en entraîner la large diffusion dans la jurisprudence arbitrale, renforçant par là-même son autorité.

Derrière ces différentes fonctions assignées à la norme soft ou doctrinale - et par extension à la jurisprudence - une motivation plus utilitariste se dessine : la référence à des normes dénuées d'autorité hard participe du discours argumentatif destiné à convaincre les parties que la solution retenue est la bonne, et, au-delà, les organes d'annulation que la sentence rendue n'est pas viciée. Le comité ad hoc de l'affaire TECO a ainsi relevé que le défaut de motifs ou l'excès manifeste de pouvoir n'étaient pas constitués dans la sentence examinée, le renvoi par les arbitres à divers commentaires doctrinaux participant de cette conclusion ${ }^{169}$.

Des motivations qui touchent à une forme de politique juridictionnelle sont également susceptibles de poindre. On sait ainsi que le recours à la soft law par la Cour européenne des droits de l'homme est mis au service d'une interprétation dynamique et évolutive de la convention dont elle est l'organe, au terme de laquelle la volonté exprimée par l'État pèse moins que la nécessité d'assurer la protection

164. CIRDI, United Utilities (Talinn) BV et Aktsiaselts Tallinna Vesi c. Estonie, ${ }^{\circ}$ ARB/14/24, décision sur la production de documents, 3 mai 2016 , $\$ 9$.

165. Ibid., $\S 10$ et s.

166. CIRDI, Hydro Srl et al. c. Albanie, $\mathrm{n}^{\circ} \mathrm{ARB} / 15 / 28$, ordonnance sur les mesures conservatoires, 3 mars 2016, §§ 3.31-3.32. Voir CPA (CNUDCI), Flemingo Dutyfree Shop Private Ltd c. Pologne, sentence, 12 août 2016, § 924 (référence aux principes Unidroit de 2010 sur les contrats commerciaux internationaux au sujet de l'indemnisation de la perte de chance).

167. CIRDI, ConocoPhillips Petrozuata BV et al. c. Venezuela, $\mathrm{n}^{\circ} \mathrm{ARB} / 07 / 30$, décision sur la demande de récusation de L. Yves Fortier, 26 juillet 2016, $\$ 12$.

168. Voir CPA (CNUDCI), Copper Mesa Mining Corporation c. Équateur, $\mathrm{n}^{\circ}$ 201-2, sentence, 15 mars $2016, \S 6.95$ où la thèse de doctorat de B. Stern est citée " en seconde main " à travers un extrait de la sentence Occidental ; CIRDI, Crystallex International Corporation c. Venezuela, ${ }^{\circ} \mathrm{ARB}(\mathrm{AF}) / 11 / 2$, sentence, 4 avril 2016, § 935 (reprise d'une décision du tribunal irano-américain des différends citant WHITEMAN, Damages in International Law).

169. Comité ad hoc CIRDI, TECO Guatemala Holdings LLC c. Guatemala, n ARB/10/23, décision sur l'annulation, 5 avril 2016, $\S \S 256,313,328$. 
effective des droits fondamentaux des individus ${ }^{170}$. On ne saurait exclure qu'un phénomène analogue se développe dans l'arbitrage d'investissement. Toutefois, la plupart des instruments contemporains de soft law visent davantage la préservation des intérêts de l'État que l'approfondissement de la protection des investisseurs. Les affaires Philip Morris et Urbaser, dans lesquelles des sources informelles ont été mobilisées aux fins de consacrer la théorie des police powers de l'État ou pour soumettre les investisseurs au droit international des droits de l'homme ${ }^{171}$, montrent ainsi que la soft law ou la doctrine peuvent être des facteurs de réaffirmation de l'État face à la personne privée, dont les droits économiques ne sauraient prévaloir sur l'intérêt général même si les États ont trop souvent « oublié » ces considérations au moment de conclure leurs traités d'investissement.

Au terme du passage en revue de la place réservée aux différentes sources du droit international dans la jurisprudence arbitrale de 2016 et de la tendance de fond qu'il révèle, il apparaît que la nomenclature traditionnelle de l'article $38 \mathrm{du}$ Statut de la CIJ - non applicable en tant que tel à l'arbitrage d'investissement mais censé refléter les différentes sources du droit international ${ }^{172}$ - pourrait être réorganisée, non pas en fonction de l'origine des règles mais de leur autorité, hard ou soft. D’un côté, la catégorie des sources principales, génératrices de règles hard, serait composée du traité applicable à chaque espèce, du droit coutumier et des principes, qu'ils soient " généraux de droit », ou tirés de la coutume voire d'une jurisprudence constante. La catégorie des sources auxiliaires, à l'origine de règles soft - i.e. exerçant à un titre ou un autre une influence sur la solution arbitrale permettrait de regrouper des règles intrinsèquement dures mais non applicables au litige (traités externes, décisions juridictionnelles - qui n'ont autorité que relative de la chose jugée) ainsi que la soft law tirée d'instruments formellement impuissants ou de la doctrine. Cette typologie fonctionnelle reflète fidèlement le rapport aux sources de droit international qui transpire de la pratique des tribunaux arbitraux d'investissement. Au reste, rien ne semble s'opposer à ce qu'elle constitue un cadre plus général de compréhension de la pratique juridictionnelle internationale.

Par ailleurs, le cru 2016 des décisions arbitrales confirme une certaine dépréciation des modes traditionnels de détermination de la norme internationale. La jurisprudence arbitrale, dont on sait qu'elle est pourtant chaotique, tend à devenir le moyen principal d'interprétation des traités et d'identification de la norme coutumière, tandis que les sources informelles acquièrent une place qu'on pourrait juger surévaluée. Le potentiel normatif des tribunaux arbitraux, notamment via les principes qu'ils consacrent, semble, de plus, encore immense. On peut s'inquiéter de cette remise en cause lancinante de certains dogmes du droit des gens ; on peut aussi trouver fort stimulante la singulière évolution de la mécanique d'un droit international plus dynamique que jamais.

170. Jean-François FLAUSS, "L'effectivité et l'efficacité de la soft law européenne dans la jurisprudence de la Cour européenne des droits de l'homme ", in J. Iliopoulos-Strangas et J.-F. Flauss (éd.), La soft law des organisations européennes, SIPE, Baden-Baden/Bern/Athènes, Nomos/Stämpfli Verlag/Ant. N. Sakkoulas Verlag, 2012, pp. 351-352. Voir notamment CEDH, Gde Ch., Demir et Baykara c. Turquie, arrêt du 12 novembre 2008, $\S 8$ 85-86.

171. CIRDI, Philip Morris Brands SARL e.a. c. Uruguay, $\mathrm{n}^{\circ} \mathrm{ARB} / 10 / 7$, sentence du 8 juillet 2016 , $\S \S 292$ et s. ; CIRDI, Urbaser SA et Consorcio de Aguas Bilbao Bizkaia, Bilbao Biskaia Ur Partzuergoa c. Argentine, $\mathrm{n}^{\circ} \mathrm{ARB} / 07 / 26$, sentence, 8 décembre 2016, $\$ \$ 1195$ et s. Voir supra, B (Coutume).

172. L'article 42 de la convention de Washington sur le droit applicable, qui est le pendant pour le CIRDI de l'article 38 du Statut de la CIJ, ne décompose pas selon leur origine les " principes de droit international » applicables. 


\section{II. - ARBITRAGE TRANSNATIONAL ET RESPONSABILITÉ INTERNATIONALE}

L'observation de la jurisprudence arbitrale de ces dernières années pouvait donner l'impression d'une tendance lourde à la marginalisation des questions de fond, tant les aspects procéduraux et contentieux semblent prendre le dessus en pratique. L'année 2016 vient néanmoins démentir cette impression générale, puisque vingt-deux sentences (rendues publiques) ont abordé ces questions, parmi lesquelles quinze ont constaté l'engagement de la responsabilité de l'État et sept l'ont rejetée, sur le fondement de différents motifs. On peut en outre préciser que parmi les sentences rendues en 2015 mais publiées cette année, trois d'entre elles ont abordé la question du fond et deux ont effectivement reconnu que la responsabilité de l'État défendeur était engagée. Il ne faut pas donner à ces chiffres plus d'importance qu'ils n'en ont, car l'engagement de la responsabilité de l'État demeure fondamentalement tributaire des circonstances de chaque affaire. Mais ils révèlent tout de même une tendance : dès lors que l'instance parvient au fond, le risque que l'engagement de la responsabilité de l'État soit reconnu est statistiquement élevé. Environ deux tiers des décisions abordant le fond se soldent ainsi par une condamnation du gouvernement ${ }^{173}$. Cela explique aussi sans aucun doute le déploiement exponentiel d'arguments procéduraux de la part des défendeurs, désireux de bloquer le procès avant que celui-ci ne parvienne au fond. Mais au-delà de ces éléments statistiques, ces chiffres traduisent un certain dynamisme du contentieux arbitral transnational et confirment le grand apport qui peut être le sien à la théorie de la responsabilité internationale. Dans la mise en œuvre de celle-ci, les tribunaux arbitraux s'appuient sans grande hésitation sur les Articles de la Commission du droit international relatifs à la responsabilité de l'État dont ils proclament sans peine la valeur coutumière, au moins sur certains points ${ }^{174}$. Il reste qu'une personnalisation à outrance de ce texte a pu être constatée, certains tribunaux en attribuant la paternité au seul J. Crawford dont le rôle fut certes important, mais dont il faut rappeler qu'il ne fut « que » le Rapporteur spécial (succédant à quatre autres) au moment de la seconde lecture du texte final, luimême fruit d'un délicat compromis ${ }^{175}$. En tout état de cause, la pratique arbitrale de l'année 2016 est riche d'enseignements à la fois sur les conditions d'engagement de la responsabilité (A) et sur les conséquences de cet engagement (B).

\section{A. Engagement de la responsabilité}

Les conditions de l'engagement de la responsabilité de l'État sont connues mais elles continuent de donner lieu à des évolutions intéressantes dans le cadre du contentieux arbitral. Les questions d'attribution, de fait composite et de causalité ont plus particulièrement été abordées cette année. La question de l'illicéité des actes étatiques est par ailleurs déterminante et les circonstances permettant d'exclure cette illicéité ont également donné lieu à de nouveaux développements.

173. En 2015, douze sentences ont abordé la question du fond parmi lesquelles huit ont reconnu que la responsabilité de l'État était engagée, voir cette chronique, cet Annuaire, 2015, p. 877.

174. Par exemple, pour le principe de l'attribution à l'État des actes de ses organes prévu à l'art. 4 : CIRDI, Joseph Houben c. Burundi, $\mathrm{n}^{\circ}$ ARB/13/7, sentence, 12 janvier 2016, § 172.

175. CPA (CNUDCI), Copper Mesa Mining Corporation c. Équateur, n² 201-2, sentence, 15 mars 2016, p. xvii. 


\section{Attribution}

Il est entendu qu'un État ne peut être reconnu responsable que des actes qui lui sont attribués mais cette question est de nature à soulever d'importantes difficultés. Il faut, à titre préalable, préciser que la question relève avant tout du fond puisqu'elle est une condition d'engagement de la responsabilité : elle ne peut éventuellement faire l'objet d'une analyse au stade de la compétence que de manière succincte dans le cadre d'un examen prima facie ${ }^{176}$. En outre, la jurisprudence a eu cette année l'occasion de rappeler que l'application des Articles de la CDI n'était que supplétive et pouvait être écartée en cas de lex specialis applicable. Tel est ainsi le cas de l'article 1103 de l'ALENA, qui règle la question des rapports entre l'État et les entreprises publiques et organise la responsabilité du premier pour les actes des secondes. Dans l'hypothèse où la question serait soulevée, elle devrait donc être traitée en vertu de cette disposition et non des articles de la CDI ${ }^{177}$. Sans doute cette position doit-elle être approuvée, mais l'on peut s'étonner qu'en l'espèce le tribunal ne l'ait appliquée qu'à l'article 5 (qui permet l'attribution à l'État des actes accomplis par des entités exerçant des prérogatives gouvernementales) et non à l'article 8 (qui permet cette attribution pour des actes accomplis sur instruction de l'État). En effet, dans le cas d'une entreprise publique, l'article 8 peut parfaitement s'avérer pertinent si bien que la décision du tribunal laisse planer une ambiguïté de ce point de vue. Mais assurément si l'article 1103 de l'ALENA est une lex specialis en matière d'attribution, elle doit l'être au regard de l'ensemble des normes du texte de la CDI ayant cet objet, article 8 compris.

La règle de l'attribution à l'État des actes de ses organes (article 4 du texte de la CDI) est sans doute celle qui est la moins contestée sur le principe. Cette disposition procède par renvoi au droit interne pour la qualification d'organe sans toutefois que cela ne règle toujours la question car il est, somme toute, relativement rare qu'une règle de droit interne prévoie explicitement qu'une entité donnée soit qualifiée d'organe de l'État. Le droit national ne fournit donc que rarement une solution clefs en mains aux tribunaux arbitraux, même si son importance demeure déterminante. Ainsi, le fait que des entités soient désignées par la loi comme des «compagnies » (corporations) jouissant d'une personnalité juridique distincte de celle de l'État peut conduire à écarter la qualification d'organe ${ }^{178}$. De même, si le droit national prévoit explicitement qu'un administrateur judiciaire représente le créancier et non l'État, il ne saurait a priori être question de le qualifier d'organe ${ }^{179}$. Il semble à l'inverse que certaines entités bénéficient automatiquement de la qualité d'organe aux yeux de certains tribunaux, à l'instar d'une banque centrale ${ }^{180}$. Dans ces différentes hypothèses, le droit interne aura donné aux tribunaux une solution presque automatique mais il se peut que la situation soit un peu plus complexe. Il a ainsi été jugé que le statut d'une entité en droit interne n'était pas toujours déterminant car en certains cas il est nécessaire de tenir compte de la nature des fonctions exer-

176. CPA (CNUDCI), Mesa Power Group, LLC c. Canada, n²012-17, sentence, 24 mars 2016, § 340.

177. Ibid., § 362 .

178. Ibid., § 343 .

179. CIRDI, MNSS BV et Recupero Credito Acciaio NV c. Monténégro, ARB(AF)/12/8, sentence, 4 mai $2016, \S 313$.

180. CIRDI, Garanti Koza LLP c. Turkménistan, n ARB/11/20, sentence, 19 décembre 2016, § 352. C'est également ce qui ressort a contrario de la sentence CIRDI, MNSS BV et Recupero Credito Acciaio NV c. Monténégro, ARB(AF)/12/8, sentence, 4 mai 2016, $\S 299$ et s. Le tribunal refuse de considérer que les actes d'une banque privée puissent être attribués à l'État sur le fondement de l'article 8, en dépit du contrôle exercé sur elle par la Banque centrale. En revanche, le tribunal examine la licéité internationale des actes de cette dernière sans questionner l'imputabilité de ses actes à l'État, qui semble donc admise sans discuter. 
cées par celle-ci ${ }^{181}$. Certains tribunaux ont jugé à cet égard que l'existence d'une personnalité juridique autonome en vertu du droit de l'État écartait en principe la qualification d'organe ${ }^{182}$. Mais l'on ne saurait en tirer de conclusion définitive : en certains cas, la question de la personnalité juridique distincte aura été sans influence déterminante. À l'extrême, il a été jugé que l'appartenance d'une entité privée - jouissant donc d'une personnalité juridique propre - à l'État constituait une présomption (simple) de qualification d'organe ${ }^{183}$. Cette position est sans doute minoritaire car si une présomption devait s'appliquer à une entité de droit privé, elle devrait être dans le sens inverse, à savoir le refus de la qualification d'organe. Cela étant dit, quel que soit le sens de la présomption, elle devrait pouvoir être renversée par la preuve contraire ${ }^{184}$. D'une manière générale d'ailleurs, au-delà du statut formel, il est entendu que la méthode du faisceau d'indices peut être déterminante pour qualifier d'organe une entité qui n'est pas explicitement désignée comme tel par les règles du droit national ${ }^{185}$. Parmi les éléments pertinents, la détention du capital par l'État, le contrôle des opérations par ce dernier, le mode de nomination des dirigeants ou le contrôle opéré par le gouvernement sont autant d'éléments permettant de trancher dans le sens ou non de la qualification d'organe.

L'importance conférée au droit interne par l'article 4 du texte de la CDI ne semble toutefois pas admise de la même manière par l'ensemble des tribunaux arbitraux. Dans l'affaire Flemingo, les arbitres ont adopté un raisonnement reléguant le droit national au second rang, endossant une position pouvant prêter à controverse. Commençant par rappeler qu'en vertu de l'article 4(2), est considérée comme un organe de l'État toute entité bénéficiant de ce statut en vertu du droit interne, le tribunal a jugé que cette affirmation n'épuisait pas la question : une entité non qualifiée d'organe en vertu du droit interne peut tout de même être considérée comme telle si un certain nombre d'éléments en ce sens sont réunis ${ }^{186}$. En soi, cette affirmation est parfaitement vraie mais la suite du raisonnement vient jeter un certain trouble. La démonstration se poursuit en effet par une référence à l'article 3 du texte de la CDI, qui rappelle que la qualification d'un fait comme licite par le droit interne n'est pas pertinente dans la qualification d'un acte comme internationalement illicite ${ }^{187}$. En réalité, cette affirmation - incontestable dans l'absolu - est sans aucun lien avec l'attribution. Elle conduit à une confusion regrettable entre le raisonnement applicable à la qualification de l'acte - qui relève strictement du droit international et est une question autonome par rapport au droit interne - et celui applicable à la notion d'organe qui continue de dépendre en partie du droit national. Une chose est de dire qu'une entité qui n'est pas formellement un organe en vertu du droit interne peut l'être en application de certains critères, une autre est de dire que la qualification d'organe est indépendante du droit national. La référence à l'article 3 pourrait donc conduire à cette seconde affirmation, qui n'est pas exacte. Cela étant dit, la volonté d'indépendance des tribunaux arbitraux sur cette question ne s'exprime pas seulement au regard du droit des États. Ainsi, la qualification de certaines entités comme «personnes publiques » (public bodies) par l’Organe de règlement des différends de l'OMC n'a pas été jugé pertinente par un

181. CPA (CNUDCI), Kristian Almås et Geir Almås c. Pologne, sentence, 27 juin 2016, § 207.

182. Ibid., § 208 .

183. CPA (CNUDCI), Flemingo Dutyfree Shop Private Ltd c. Pologne, sentence, 12 août 2016, § 426.

184. Dans l'affaire Flemingo, loin d'être renversée, la présomption a été confirmée par un certain nombre d'indices. Le tribunal n'a donc éprouvé aucune difficulté à qualifier d'organe de l'État une entité de droit privé, même s'il s'agissait en l'occurrence d'un organe de facto, voir le $§ 435$.

185. Outre Flemingo, $\$ 426$ et s., voir CIRDI, Tenaris SA et Talta-Trading e Marketing Sociedade Unipessoal Lda. c. Venezuela, $\mathrm{n}^{\circ} \mathrm{ARB} / 12 / 23$, sentence, 29 janvier 2016, $\S \S 412-413$ où le faisceau d'indices conduira le tribunal à refuser la qualification d'organe.

186. Flemingo Dutyfree Shop Private Ltd c. Pologne, § 433.

187. Ibid. 
tribunal arbitral dans la mesure où cette qualification reposait sur un autre texte et qu'il ne saurait donc en être tiré de conséquence automatique dans le cadre des Articles de la CDI ${ }^{188}$. Il semble tout de même que, à rebours de ces deux solutions, le droit interne comme les qualifications retenues par les autres juridictions internationales, sans lier les arbitres, puissent être des éléments pertinents dans le cadre d'un raisonnement fondé sur la méthode du faisceau d'indices.

On sait d'ailleurs que les Articles attribuent à l'État non seulement les actes de ses organes mais aussi ceux des personnes ou entités qui, sans avoir cette qualité, exercent des prérogatives de puissance publique. Le critère est alors différent de celui applicable à l'organe et relève de l'article 5 du texte de la CDI, mais les tribunaux ne font pas toujours preuve d'une rigueur exemplaire en la matière. Ainsi dans l'affaire İckale İnşaat Limited Şirketi c. Turkménistan, le tribunal avait jugé au sujet d'une banque qu'elle était " an entity separate from the State and cannot be considered as a State organ capable of exercising governmental authority, or puissance publique " ${ }^{189}$. Cette formule est de nature à induire en erreur, car elle semble confondre deux critères : celui de l'organe (art. 4) et celui de l'entité habilitée à exercer des prérogatives de puissance publique (art. 5). Puisqu'il s'agit de deux hypothèses distinctes, le critère des prérogatives de puissance publique n'a pas, en principe, à s'appliquer dans l'hypothèse d'un organe de l'État identifié comme tel. Il ne s'agit donc pas ici de dire qu'il y a confusion, mais que la formule retenue par la sentence n'est pas des plus heureuses. C'est d'ailleurs un reproche similaire que l'on peut adresser à la sentence Flemingo sur ce point : ayant considéré que, en vertu d'un ensemble d'indices, la société PPL possédée par l'État devait être qualifiée d'organe de facto de ce dernier, le tribunal a poursuivi son raisonnement en indiquant qu'il aurait pu tout aussi bien se placer sous l'angle de l'article 5 puisque la société exerçait des prérogatives gouvernementales ${ }^{190}$. Le tribunal opère bien ici une distinction entre les deux dispositions et les régimes juridiques qui leur sont applicables mais l'on peine à voir en quoi les développements sur le fondement de l'article 5 étaient nécessaires à partir du moment où la qualification d'organe était acquise. De tels développements, que le tribunal qualifie lui-même de surabondants ${ }^{191}$, ne peuvent que venir provoquer la confusion en laissant croire que la qualification d'organe n'est pas suffisante et qu'il faudrait y adjoindre le critère de l'exercice de prérogatives de puissance publique.

Ce dernier a pu cependant donner lieu à d'intéressants développements dans le cadre de l'article 5. Dans l'affaire Kristian Almås c. Pologne, constatant que l'entité à l'origine des actes attaqués ne pouvait être qualifiée d'organe de l'État, le tribunal s'est ainsi placé sous l'angle de l'article 5 afin de vérifier si elle avait exercé des prérogatives gouvernementales. Cette analyse suppose donc d'examiner en détail les actes reprochés afin de savoir s'ils mobilisent des pouvoirs particuliers justifiant leur imputabilité à l'État. En l'espèce, l'acte en question était une rupture du contrat passé entre l'entité en question et l'investisseur, si bien que la question contractuelle s'est invitée dans le traitement du problème de l'imputation. Pour trancher ce dernier, le tribunal s'est donc mué en juge du contrat afin de déterminer si la rupture reprochée avait été déclenchée en vertu de pouvoirs contractuellement reconnus ou en application de prérogatives telles qu'elles révéleraient une nature étatique ${ }^{192}$. Au terme de l'analyse, ayant jugé que les pouvoirs étaient de nature contractuelle, le tribunal décida donc que l'acte était celui d'un cocontractant et qu'il

188. CPA (CNUDCI), Mesa Power Group, LLC c. Canada, n 2012-17, sentence, 24 mars 2016, § 346. $\S 370$.

189. CIRDI, İçkale İşaat Limited Şirketi c. Turkménistan, $\mathrm{n}^{\circ} \mathrm{ARB} / 10 / 24$, sentence, 8 mars 2016,

190. Flemingo Dutyfree Shop Private Ltd c. Pologne, §§ 436-446.

191. Ibid., § 436.

192. CPA (CNUDCI), Kristian Almås et Geir Almås c. Pologne, sentence, 27 juin 2016, $\S \S 224$ et s. 
ne pouvait, comme tel, être imputé à l'État ${ }^{193}$. Cette position se trouvait confortée par le fait qu'aucun motif politique extracontractuel ne pouvait en l'espèce être identifié pour justifier la violation ${ }^{194}$. Il est donc intéressant de voir ici se déployer dans le cadre de l'imputation des analyses ordinairement réservées aux questions liées à la distinction entre treaty et contract claims, qui relève de la problématique générale de la compétence des tribunaux arbitraux ${ }^{195}$. C'est le signe que la nature des actes accomplis dans le cadre d'un contrat n'est pas seulement pertinente en termes de compétence, elle peut l'être également en termes d'imputation. Mais dans les deux cas, elle est susceptible d'empêcher l'examen de la demande au fond.

\section{Fait composite}

La question du fait composite est régulièrement abordée dans la jurisprudence arbitrale, en règle générale - et assez logiquement - à l'occasion d'analyses liées à l'expropriation indirecte, et singulièrement à l'expropriation " rampante ». La notion est en effet particulièrement pertinente si l'on rappelle que l'article 15 des Articles de la CDI la définit comme « la violation d'une obligation internationale par l'État à raison d'une série d'actions ou d'omissions, définie dans son ensemble comme illicite ». Cette définition générale paraît presque exactement calquée sur celle de l'expropriation rampante (creeping expropriation), impliquant une série d'actes de l'État dont aucun n'est illicite en lui-même mais dont l'accumulation équivaut à une dépossession de l'investisseur ${ }^{196}$. Le fait composite est donc un outil d'analyse efficace à cet égard et confirme parfaitement que la responsabilité de l'État en matière d'investissement s'inscrit pleinement dans le cadre conceptuel de la responsabilité de l'État en droit international général. La qualification de l'expropriation rampante comme fait composite a du reste été confirmée par la jurisprudence en $2016{ }^{197}$. C'est donc bien l'effet cumulé qui constitue l'expropriation, à partir du moment où cet effet a privé l'investissement de sa valeur ${ }^{198}$. Mais la difficulté, en pareil cas, est celle de l'articulation avec la compétence temporelle de la juridiction : la question, relativement classique en la matière, se pose parce que les agissements dont l'accumulation constitue l'acte illicite se déploient nécessairement dans le temps et il est possible que certains d'entre eux aient été accomplis avant que la norme dont la violation est alléguée ne soit entrée en vigueur. En pareil cas la qualification de fait composite est déterminante car elle peut avoir pour effet de fonder malgré tout la compétence du tribunal arbitral même si celleci ne pourra s'exercer que sur les faits relevant effectivement du champ temporel de cette compétence ${ }^{199}$.

Il importe cependant de préciser que la notion de fait composite n'est pas exclusivement pertinente pour le cas de l'expropriation. Elle a pu être également employée dans le contexte du traitement juste et équitable (TJE), dont le contenu est lui-même suffisamment indéfini pour s'adapter à ce type de concept. Dans

193. Ibid., § 251 .

194. Ibid., $\S \$ 258-261$.

195. Voir sur la question en général not. Ibrahim FADLALLAH, « La distinction Treaty claims - Contract claims ", in Charles LEBEN (dir.), Droit international des investissements et de l'arbitrage transnational, Paris, Pedone, 2015, pp. 759-772.

196. Voir par exemple Rumeli Telekom A.S. and Telsim Mobil Telekomikasyon Hizmetleri A.S. c. Kazaghstan, $\mathrm{n}^{\circ} \mathrm{ARB} / 05 / 16,29$ juillet 2008, $\$ 684$.

197. CIRDI, Crystallex International Corporation c. Venezuela, $\mathrm{n}^{\circ} \mathrm{ARB}(\mathrm{AF}) / 11 / 2$, sentence, 4 avril 2016, § 669. Voir également, sans citer les Articles de la CDI mais reprenant exactement la définition : Trib. ad hoc (CNUDCI), Oxus c. Ouzbékistan, sentence finale, 17 décembre 2015, § 740.

198. Crystallex International Corporation c. Venezuela, $\$ 708$.

199. Voir CIRDI, Spence International Investments, LLC et al. c. Costa Rica, $\mathrm{n}^{\circ} \mathrm{UNCT} / 13 / 2$, sentence intérimaire, 25 octobre 2016. 
l'affaire Pey Casado, le tribunal avait ainsi identifié un lien étroit entre les deux notions : pour lui, c'est un ensemble de comportements qui, cumulés, permettaient d'identifier une violation du TJE en l'espèce, ce qui « correspond au cas typique en matière de protection des investissements, étant donné que le concept de 'traitement' dans son ensemble est un concept qui peut se référer et se réfère effectivement souvent à un ensemble de comportements ${ }^{200}$. D'autres tribunaux ont d'ailleurs admis la pertinence du fait composite pour analyser une violation du traitement juste et équitable, considérant qu'en la matière, c'est l'ensemble du comportement de l'État qui devait être pris en considération et pas nécessairement chaque acte pris isolément ${ }^{201}$. Cette manière d'aborder le traitement juste et équitable vient très clairement confirmer qu'il s'agit d'un standard dont le contenu ne peut être fixé à l'avance et qui ne peut être appliqué que dans un contexte particulier ${ }^{202}$. De ce point de vue, il reste très largement tributaire de la sensibilité du tribunal arbitral chargé de l'appliquer, ce que l'on peut regretter. Cette situation explique d'ailleurs sans doute la tendance conventionnelle récente à l'abandon de la mention de "traitement juste et équitable " pour lui préférer une liste d'obligations plus précises telles que l'interdiction du déni de justice ou de la discrimination ${ }^{203}$.

\section{Dommage et lien de causalité}

La question du lien de causalité est rarement évoquée isolément dans le droit de la responsabilité internationale. L'une des principales explications tient sans aucun doute à la rédaction particulière de l'article $1^{\text {er }}$ du texte de la CDI, qui ne pose que deux conditions à l'engagement de la responsabilité de l'État : d'une part un fait internationalement illicite, d'autre part l'imputabilité de ce fait à un État. En l'appliquant à la lettre, il n'est pas question de causalité puisque celle-ci désigne le lien entre le fait illicite et le dommage causé. À partir du moment où ce dernier n'est pas formellement une condition d'engagement de la responsabilité, la causalité ne saurait l'être davantage. Le lien de causalité prend, en revanche, toute son importance au stade de la réparation qui se définit strictement par rapport au dommage causé si dommage il y a l'État ne peut être condamné à réparer que le préjudice qu'il a provoqué par son acte illicite. Il apparaît donc que le lien de causalité ne saurait être une condition d'engagement de la responsabilité, mais qu'il est essentiel une fois cette responsabilité engagée.

En réalité, les tribunaux arbitraux ne font pas preuve d'une rigueur exemplaire en la matière mais il est vrai que leur situation n'est guère confortable. Le droit de la responsabilité internationale a d'abord été pensé dans un cadre interétatique et si l'application de la plupart de ses normes à des relations transnationales n'est plus véritablement contestée aujourd'hui ${ }^{204}$, un certain décalage subsiste sur certains points. C'est en particulier le cas du dommage, qui n'est sans doute pas déterminant dans le contentieux interétatique mais demeure absolument central dans celui qui oppose investisseurs étrangers et États. Même si les tribunaux s'en tiennent pour la majorité à une certaine orthodoxie en écartant le dommage - et donc le

200. CIRDI, Victor Pey Casado et President Allende Foundation c. Chili, $\mathrm{n}^{\circ} \mathrm{ARB} / 98 / 2$, sentence, 13 septembre 2016, § 209.

201. Crystallex International Corporation c. Venezuela, $\S 545$.

202. Voir sur cette question Yves NouVEL, "Traitement juste et équitable, sécurité pleine et entière ", in Charles LEBEN (dir.), Droit international des investissements et de l'arbitrage transnational, op. cit., pp. 287-345, spé. pp. 299-303.

203. C'est notamment le cas de l'article 8.10 de l'Accord économique et commercial global (CETA selon son acronyme anglais) signé le 30 octobre 2016 entre le Canada et l'Union européenne.

204. Pour une réaffirmation cette année : CIRDI, Vestey Group Limited c. Venezuela, n ARB/06/4, sentence, 15 avril $2016, \S 326$. 
lien de causalité - des conditions d'engagement de la responsabilité, l'importance de la réparation dans le contentieux transnational les conduit naturellement à commettre parfois quelques écarts ${ }^{205}$.

On voit d'ailleurs difficilement comment il pourrait en être autrement dans certains cas, notamment dans le cadre du contentieux de l'expropriation qui n'est pas franchement marginal en droit des investissements. Dès lors en effet qu'un investisseur estime avoir été victime d'une expropriation illicite, le tribunal arbitral est tenu de vérifier s'il y a eu dépossession et si, le cas échéant, cette dépossession est liée à un acte de l'État. Compte tenu de l'importance de la dépossession dans le droit de l'expropriation, il est possible de dire que le lien de causalité est « inhérent » à cette notion ${ }^{206}$. Cela peut expliquer qu'en certaines affaires, le dommage et le lien de causalité aient été traités au stade de l'établissement de la responsabilité et non au moment de la réparation même s'il est vrai qu'en toute rigueur ce n'est pas la méthode prévue par les Articles de la CDI. Ainsi dans l'affaire Peter Allard c. Barbade, l'investisseur avait conçu et financé un complexe d'écotourisme et se plaignait de ce que l'État, en n'adoptant pas une réglementation environnementale suffisamment ferme, avait laissé l'écosystème se dégrader et avait donc privé son investissement de toute valeur. On peut noter au passage l'inversion du schéma classique, le demandeur ne se plaignant pas d'une mesure environnementale mais de l'absence d'une telle mesure pour expliquer sa déconvenue économique. Mais une telle demande supposait nécessairement de rechercher le lien entre l'effet provoqué sur l'investissement - que l'investisseur estimait être équivalent à une expropriation - et l'action ou le défaut d'action reproché à l'État. Cela peut expliquer que le tribunal ait débuté son analyse par la recherche d'un dommage avant de voir si celui-ci pouvait être lié au comportement des autorités ${ }^{207}$. Il est difficile de dire, à la lecture de la sentence, si c'est l'absence de dommage ou l'absence de lien qui aura justifié le rejet des prétentions de l'investisseur par le tribunal mais cela montre que la causalité peut être appelée, en fonction des espèces, à jouer un rôle en amont de la question de la réparation. De manière plus diffuse, des éléments de raisonnement similaires peuvent d'ailleurs être identifiés dans la sentence Urbaser c. Argentine, dans laquelle le tribunal arbitral n'a pas hésité, au stade de l'établissement de la responsabilité, à rechercher s'il existait un lien de causalité entre le dommage subi par l'investisseur, établi par ailleurs, et la crise économique de 2001 ainsi que les mesures adoptées par le gouvernement argentin pour y faire face ${ }^{208}$. Mais le contexte très particulier du contentieux argentin, qui n'est pas encore totalement soldé, explique sans doute cette entorse à la stricte orthodoxie.

\section{Circonstances excluant l'illicéité : le retour de l'état de nécessité}

On se souvient que c'est dans le cadre de ce même contentieux que l'état de nécessité a connu ses développements les plus remarquables, même si la jurisprudence de l'année passée a permis de montrer qu'il n'y était pas cantonné ${ }^{209}$. L'année 2016 aura de ce point de vue été marquée par le retour de cette notion dans le contentieux impliquant le gouvernement argentin, sans doute du reste à la

205. Sur cette question voir Franck LATTY, «Conditions de l'engagement de la responsabilité de l'État ", in Charles LEBEN (dir.), Droit international des investissements et de l'arbitrage transnational, op. cit., pp. 415-461, spéc. pp. 417-420.

206. Voir CIRDI, Joseph Houben c. Burundi, n ARB/13/7, sentence, 12 janvier 2016, § 217.

207. CPA (CNUDCI), Peter A. Allard c. Barbade, ${ }^{\circ} 2012-06$, sentence, 27 juin 2016, $\$ \$ 80$ et s.

208. CIRDI, Urbaser SA et Consorcio de Aguas Bilbao Bizkaia, Bilbao Biskaia Ur Partzuergoa c. Argentine, $\mathrm{n}^{\circ} \mathrm{ARB} / 07 / 26$, sentence, 8 décembre 2016 , not. $\$ 534$.

209. Voir cette chronique, cet Annuaire 2015, p. 882, au sujet de l'état de nécessité invoqué dans le contentieux impliquant les réformes agraires au Zimbabwe. 
plus grande satisfaction de ce dernier. Dans la même affaire Urbaser, le tribunal a en effet admis, comme d'autres avant mais sans que cette tendance ne puisse être jugée majoritaire ${ }^{210}$, que l'Argentine s'était trouvée en situation de nécessité pendant l'année $2002^{211}$. Le tribunal n'a pas ignoré que la politique du gouvernement avait contribué à la survenance de la crise, mais cette circonstance n'a pas été jugée suffisante pour inverser la solution : d'une part parce que, pour établie qu'elle soit, cette contribution n'était pas volontaire ; d'autre part parce qu'il eût été impossible dans un premier temps de réaliser que les mesures gouvernementales étaient de nature à avoir cet impact ; enfin parce que ces mesures n'étaient qu'un élément expliquant la crise mais que les causes de celle-ci étaient aussi et surtout exogènes ${ }^{212}$. Cette solution doit sans doute être saluée même si elle est loin de clore la comédie dramatique qui se joue autour de cette question : quelques mois avant cette sentence, deux comités $a d$ hoc avaient en effet refusé dans deux autres affaires d'annuler les sentences qui leur étaient déférées, considérant que les tribunaux avaient effectivement examiné les conditions de l'article 25 du texte de la CDI et avaient correctement conclu que l'Argentine n'était pas en situation de nécessité ${ }^{213}$. Sans doute d'ailleurs la solution adoptée dans l'affaire Urbaser souhaite-t-elle dépasser ces contradictions jurisprudentielles en retenant une position empreinte de nuances. Il n'est pas impossible en effet que les tribunaux ayant rejeté l'état de nécessité l'aient fait pour ne pas exonérer l'Argentine de sa responsabilité, partant du principe que celle-ci demeure partiellement établie. Or, la sentence Urbaser ne reconnaît la situation de nécessité que sur une période relativement brève, loin de couvrir la totalité du contentieux. Ce faisant, les arbitres s'inscrivent dans une ligne de pensée très raisonnable consistant à voir dans l'état de nécessité une cause d'atténuation plutôt que d'exclusion de la responsabilité. Formellement, c'est bien d'une exclusion qu'il s'agit mais en la réduisant à quelques mois, le tribunal permet de maintenir le principe même d'une responsabilité de l'Argentine tout en réduisant son obligation de réparation à proportion de ce dont elle est effectivement responsable. On doit simplement signaler qu'en l'espèce, n'ayant identifié aucun dommage causé par le fait illicite, le tribunal ne prononcera aucune réparation. Mais sa position de principe n'en demeure pas moins d'un très grand intérêt.

Une telle approche, qui n'est pas totalement inédite dans le contexte argentin ${ }^{214}$, permet de tenir compte de la situation délicate du pays sans pour autant faire peser uniquement sur l'investisseur la charge des conséquences de la crise. Admettre l'effet «atténuateur » et non pas exclusif de la nécessité permettrait d'ailleurs d'expliquer juridiquement pourquoi, en vertu des Articles de la CDI et du droit coutumier, l'obligation d'indemnisation des pertes effectives peut demeurer à la charge de l'État quand bien même une situation de nécessité serait reconnue ${ }^{215}$. Dans l'absolu, rien ne justifie une telle obligation : l'État n'est pas un assureur des

210. Dans le cadre du contentieux argentin, l'état de nécessité avait été admis dans les sentences Continental Casualty Company c. Argentine, $\mathrm{n}^{\circ} \mathrm{ARB} / 03 / 9,5$ septembre 2008 et LG\&E Energy corp. LG\&E Capital, LG\&E International inc. c. Argentine, $\mathrm{n}^{\circ} \mathrm{ARB} / 02 / 1,3$ octobre 2006. En revanche il n'a pas été reconnu notamment dans les décisions CMS Gas transmission company c. Argentine, $\mathrm{n}^{\circ} \mathrm{ARB} / 01 / 8$, sentence du 12 mai 2005, Enron Corporation Ponderosa Assets, L.P. c. Argentine, n ${ }^{\circ}$ ARB/01/3, 22 mai 2007, ou Sempra Energy c. Argentine, n ARB/02/16, sentence du 28 septembre 2007, § 350.

211. Urbaser SA et Consorcio de Aguas Bilbao Bizkaia, Bilbao Biskaia Ur Partzuergoa c. Argentine, $\S \S 718-719$.

212. Ibid., $\$ \$ 710$ et $\mathrm{s}$.

213. Comité ad hoc CIRDI, Total SA c. Argentine, $\mathrm{n}^{\circ} \mathrm{ARB} / 04 / 01$, décision sur l'annulation, $1^{\mathrm{er}}$ février 2016, § 239 ; Comité ad hoc CIRDI, EDF SA et SAUR International SA c. Argentine, n ARB/03/23, décision sur l'annulation, 5 février 2016, $\S 333$ et s.

214. Elle avait déjà été retenue dans l'affaire Continental Casualty Company c. Argentine, not. § 233.

215. Cette possibilité est traduite à l'article 27 : «L'invocation d'une circonstance excluant l'illicéité conformément au présent chapitre est sans préjudice : [... b) De la question de l'indemnisation de toute perte effective causée par le fait en question". 
pertes subies en raison des évolutions de la conjoncture économique. Si nécessité il y a, donc, ce n'est pas sur lui que devrait peser la charge des mesures adoptées pour y répondre, sauf à admettre une responsabilité "sans faute " (i.e., sans acte internationalement illicite) en droit international. Mais c'est précisément si l'on admet que la nécessité atténue sa responsabilité que celle-ci demeure établie dans son principe : en pareille hypothèse, l'obligation d'indemnisation de l'État continue de reposer sur l'engagement de sa responsabilité, même si cette obligation se trouvera atténuée par la circonstance de nécessité ${ }^{216}$. De ce point de vue, cette approche doit sans doute être approuvée même si elle conduit à introduire dans le jugement une évidente part de subjectivité : déterminer une date de fin de la période de nécessité ne peut en effet être totalement exempt d'une part d'artifice. Pour les arbitres, il y a là un levier important puisque l'ampleur de la responsabilité de l'État sera inversement proportionnelle à la durée de l'état de nécessité. Dans le cas d'une situation aussi complexe que celle de l'Argentine, dans laquelle même les économistes sont en désaccord sur les origines exactes de la crise, il apparaît donc que cette détermination ne saurait reposer sur des données pleinement objectives. Mais puisque l'on admet déjà une importante part de discrétion au profit des tribunaux arbitraux dans l'engagement de la responsabilité de l'État, notamment dans l'appréciation de la violation de dispositions particulièrement floues, on ne voit pas pourquoi la même chose ne serait pas admise dès lors qu'il s'agit à l'inverse de réduire l'étendue de cette responsabilité.

\section{Protection de la liberté normative de l'État}

Parmi les critiques les plus régulièrement adressées à l'arbitrage transnational, l'une des plus fréquentes et des plus virulentes repose sur le risque qu'il ferait peser sur la capacité des États à adopter des mesures dans le sens de l'intérêt général. Mais le droit de la responsabilité offre également à ces derniers des possibilités de défendre les mesures légitimes qu'ils adoptent. Les Articles de la CDI proposent ainsi un certain nombre de circonstances excluant l'illicéité mais il est possible également de rappeler qu'il existe, en droit international général, un principe fondamental suivant lequel la responsabilité d'un État ne saurait être engagée pour une mesure poursuivant un intérêt public légitime quand bien même elle affecterait les droits d'un étranger ${ }^{217}$. Les rapports entretenus par cette "police power exception » avec les circonstances d'exclusion de l'illicite ne sont pas très clairs mais il semble que les deux notions procèdent d'une logique différente car une mesure de police power n'est aucunement illicite dans l'absolu, si bien qu'il n'est pas nécessaire d'avoir recours à une circonstance particulière pour lui « rendre » sa licéité. Quoi qu'il en soit, cette exception, largement ancrée dans le droit contemporain des investissements, a été l'occasion pour les tribunaux de rappeler que la liberté normative de l'État constitue pour eux un paramètre de première importance conduisant parfois à refuser de constater l'engagement de la responsabilité, notamment dans des affaires particulièrement sensibles. On sait en particulier que les demandes qui avaient été présentées par la société Philip Morris contre l'imposition du paquet de cigarettes «neutre » dans un certain nombre d'États ont donné lieu à de vives inquiétudes. Or, il apparaît que celles-ci n'étaient guère justifiées puisque les demandes présentées contre l'Australie et l'Uruguay ont été rejetées. On ne

216. Sur l'effet «atténuateur» de la nécessité sur la réparation, voir Sarah CASSELLA, L'état de nécessité en droit international. De l'état de nécessité aux situations de nécessité, Leiden, Nijhoff, 2011, pp. 445 et s. ; Théodore CHRISTAKIS, "Nécessité n'a pas de loi'? La nécessité en droit international ", in SFDI, La nécessité en droit international, Paris, Pedone, 2007, pp. 11-63, spé. pp. 51-54.

217. Voir par exemple Ian BRownLIE " General Course on Public International Law ", Recueil des Cours, 1995, vol. 255, pp. 9-228, p. 146. 
s'attardera pas ici sur la première qui fut réglée au stade de la compétence, pour insister sur la seconde. Dans cette affaire, le requérant estimait avoir été victime à la fois d'une expropriation et d'une violation du traitement juste et équitable mais les deux demandes furent rejetées au fond. Le raisonnement du tribunal pour y parvenir n'est pas particulièrement original et paraît pleinement recevable sur le plan juridique. Mais il traduit très clairement une volonté des arbitres de répondre aux critiques adressées à l'arbitrage, singulièrement dans le cadre de ces affaires. S'agissant ainsi de l'expropriation, le motif du rejet est tout à fait classique : le tribunal estime que la société Philip Morris n'a pas fait état d'une dépossession substantielle ${ }^{218}$. Ce motif aurait été à lui seul suffisant pour rejeter la demande, mais le tribunal a tenu à ajouter que la mesure en question relevait, en tout état de cause, de l'exercice légitime du police power de l'État ${ }^{219}$. Cette doctrine bien connue du droit international de l'expropriation postule que certaines mesures étatiques poursuivant un intérêt supérieur n'ouvrent pas droit à compensation quand bien même elles affecteraient les intérêts économiques des investisseurs étrangers. Elle a été parfois mise en œuvre par la jurisprudence dans le cas de mesures de protection de l'environnement ${ }^{220}$, de lutte contre la corruption ${ }^{221}$ ou de redressement économique ${ }^{222}$. Ici, il ne fait pas de doute qu'une mesure de protection de la santé publique relève de cette catégorie, et l'insistance du tribunal à ce sujet ne peut que s'expliquer par le contexte médiatique de l'affaire. Il s'agissait aussi, probablement, de ne pas laisser sans réponse certains arguments de la société demanderesse qui n'étaient pas tous un exemple de parfaite bonne foi, comme notamment l'allégation que la mesure contestée ne reposait pas sur des données scientifiques fiables et que la seule mention du degré de force des cigarettes pouvait permettre d'atteindre le même objectif que celui poursuivi par la réglementation ${ }^{223}$. Les arbitres insistent donc sur la reconnaissance généralisée de la doctrine des police powers en droit international général et dans l'arbitrage transnational plus particulièrement depuis quelques années ${ }^{224}$. La sentence s'appuie même sur la rédaction de l'Accord global économique et commercial (CETA) signé entre l'Union européenne et le Canada pour confirmer qu'il s'agit d'une tendance récente et marquée du droit international des investissements ${ }^{225}$. Le fait, par ailleurs, que la mesure réponde à une obligation à la fois constitutionnelle et internationale de l'État - celui-ci ayant adhéré à la convention de l'OMS sur le contrôle du tabac en 2005 - était de nature à renforcer la licéité de la mesure. De ce point de vue, sans apporter de véritable nouveauté à la doctrine des police powers en droit international, cette sentence vient très utilement rappeler ses tenants et aboutissants en même temps qu'elle est de nature à faire taire certaines critiques.

Sans employer strictement le même raisonnement au sujet du traitement juste et équitable, le tribunal arbitral s'est tout de même placé dans une perspective similaire. Ici, c'est précisément la nature de la mesure, son objectif légitime et sa prévisibilité qui ont conduit les arbitres à rejeter la demande, convoquant au passage la doctrine de la marge nationale d'appréciation développée par la Cour européenne des droits de l'homme ${ }^{226}$. La démonstration au sujet du caractère légi-

218. CIRDI, Philip Morris Brands SARL, Philip Morris Products SA c. Uruguay, n ARB/10/7, sentence, 8 juillet 2016 , spéc. $\$ \S 284$ et $\mathrm{s}$.

219. Ibid., §§ 287 et s. Voir aussi supra, I, B (Coutume).

220. Par exemple : CNUDCI, Chemtura corp. c. Canada, 2 août 2010, $\$ 266$.

221. CIRDI, EDF (Services) Ltd c. Roumanie, ARB/05/13, 8 octobre 2009, § 308.

222. CIRDI, Suez, e.a. c. Argentine, ARB/03/19, sentence au fond du 30 juillet 2010 et CNUDCI, $A W G$

Group c. Argentine, 30 juillet 2010, affaires jointes, $\S 140$.

223. Voir Philip Morris Brands SARL, Philip Morris Products SA c. Uruguay, §§ 392 et 403.

224. Ibid., $\S 291$ et s.

225. Ibid., § 300.

226. Ibid., § 399. 
time et proportionné de la mesure est ici déterminante puisqu'elle constitue l'unique motif de rejet de la plainte, contrairement à la demande fondée sur l'expropriation pour laquelle le recours aux police powers était en réalité surabondant. En matière de traitement juste et équitable, c'est bien la légitimité de la mesure qui aura guidé la décision du tribunal ${ }^{227}$. Cette décision s'inscrit donc dans la continuité d'un courant jurisprudentiel bien identifié tendant depuis quelques années à ménager la liberté normative de l'État dès lors que ce dernier n'en abuse pas.

\section{B. Contenu de la responsabilité}

Plusieurs questions ont été abordées dans la jurisprudence récente au sujet des suites de l'engagement de la responsabilité, permettant de préciser certains aspects du droit international à ce sujet. Avant d'aborder la question du standard de réparation, toujours complexe, la question du comportement de la victime et celle du dommage moral doivent être évoquées.

\section{Prise en compte du comportement de la victime}

Le rééquilibrage général du droit de l'investissement au profit des États observé depuis quelques années maintenant ne se traduit pas seulement au stade de l'édiction des règles mais également dans les conditions et les conséquences de l'engagement de la responsabilité de ceux-ci. Une fois cette dernière établie en effet, la réparation est généralement l'occasion de tenir compte de paramètres ordinairement exclus du raisonnement, à commencer par le comportement de l'investisseur ${ }^{228}$. Les tribunaux arbitraux n'éprouvent donc plus guère d'hésitation à réduire le montant de l'indemnisation à proportion de la participation de l'investisseur au dommage ou à la situation qui l'aura provoqué. Cette possibilité semble acceptée sur le principe ${ }^{229}$, et ne semble pas devoir être questionnée. Dans le prolongement de cette idée, il est d'ailleurs entendu que l'État ne saurait être responsable d'actes qui ne sont pas la cause mais la conséquence de la (mauvaise) situation dans laquelle se trouve l'investisseur ${ }^{230}$. Cette possibilité de tenir compte de tout événement extérieur à l'État et ayant participé au dommage, qui résulte du droit international général, est aujourd'hui largement appliquée par les tribunaux arbitraux ${ }^{231}$. C'est aussi parce que le droit international des investissements ne joue pas à la manière d'une assurance des opérations économiques transnationales que tous les éléments du contexte ayant causé le préjudice autre que le ou les actes illicites doivent être pris en compte au stade de la réparation ${ }^{232}$.

227. Voir en particulier les $\S$ 401-434.

228. Il est vrai aussi que ce comportement peut être sanctionné à un stade préliminaire notamment s'il constitue une violation du droit local de l'État d'accueil. Sur la question générale de la prise en compte du comportement de l'investisseur, voir Inès EL HAYEK, La prise en compte du comportement de l'investisseur dans le cadre de l'arbitrage fondé sur les traités d'investissement, thèse université Paris 1, 2016, multigr., $493 \mathrm{p}$.

229. Voir à cet égard la remarque du tribunal dans CIRDI, Garanti Koza LLP c. Turkménistan, $\mathrm{ARB} / 11 / 20$, sentence, 19 décembre 2016, § 431.

230. Trib. ad hoc (CNUDCI), Oxus c. Ouzbékistan, sentence finale, 17 décembre 2015, § 826.

231. L'article 39 du texte de la CDI établit ainsi très clairement que « [p] our déterminer la réparation, il est tenu compte de la contribution au préjudice due à l'action ou à l'omission, intentionnelle ou par négligence, de l'État lésé ou de toute personne ou entité au titre de laquelle réparation est demandée ". Sur cette question, voir en général A. MOUTIER-LOPET, "Contribution to the Injury », in James CRAWFORD, Alain PELLET et Simon OLLESON (dir.), The Law of International Responsibility, Oxford University Press, 2010, pp. 639-645.

232. CIRDI, Hochtief AG c. Argentine, n ARB/07/31, sentence, 19 décembre 2016, §§ 21-22. 
Il se peut néanmoins que l'application de ces principes conduise à des solutions discutables, au moins de prime abord. Dans l'affaire Copper Mesa Mining c. Equateur, le tribunal semble ainsi avoir jugé que la doctrine des " mains propres » se confondait avec la possibilité de tenir compte du comportement de la victime dans la fixation du montant de la réparation, tel qu'il est prévu à l'article 39 du texte de la CDI ${ }^{233}$. Une telle assimilation peut sembler peu rigoureuse puisque la doctrine des mains propres - pour autant qu'elle soit établie en droit international des investissements - ou même la question de la conformité de l'investissement au droit local relèvent sans doute davantage de la recevabilité que du fond ${ }^{234}$ alors que la prise en compte de la participation de l'investisseur au préjudice ne peut être examinée qu'au moment de la détermination de la réparation. Néanmoins l'incertitude qui entoure encore ces questions complexes doit conduire à la plus grande prudence. Il peut en effet être soutenu que l'examen des "mains propres » ou de la conformité de l'investissement au droit local devrait être mené au fond, au nom de l'égalité entre les parties : puisque c'est au fond que la licéité du comportement étatique est analysée, c'est au même stade que la licéité des agissements de l'investisseur devrait l'être également ${ }^{235}$. Même si cette position pourrait être discutée, elle peut aussi s'expliquer par un certain pragmatisme et une volonté de simplicité : plutôt que de construire des théories complexes autour de la recevabilité, rien n'empêche un tribunal arbitral d'accepter d'examiner une affaire au fond et, éventuellement, de refuser toute indemnisation au profit de l'investisseur si la contribution de ce dernier à son propre dommage est déterminante. Ce point de vue a été exprimé très tôt dans la pratique contentieuse internationale puisque dans l'affaire du Chemin de fer de la baie de Delagoa, le tribunal indiquait que "toutes [les] circonstances qui peuvent être alléguées à la charge de la compagnie concessionnaire et à la décharge du gouvernement portugais atténuent la responsabilité de ce dernier et justifient [...] une réduction de la réparation à allouer ${ }^{236}$. Sur le principe, donc, le fait de tenir compte du comportement de la victime au stade de la réparation peut être justifié même si l'assimilation d'une telle approche à la doctrine des mains propres peut être discutée. Il n'en reste pas moins que cette position peut conduire à conférer au tribunal arbitral un pouvoir quasi-discrétionnaire : dans l'affaire Copper Mesa Mining, les arbitres ont estimé que le requérant avait contribué à $30 \%$ à la survenance du dommage et que son indemnisation devait être réduite d'autant ${ }^{237}$. La solution est un peu trop idéale pour être véritablement crédible. $\mathrm{Au}$ reste, l'on serait bien en peine d'expliquer sur quoi repose exactement ce chiffre de $30 \%$. Manifestement, il s'agit d'une appréciation globale procédant davantage d'un sentiment général des arbitres (une « intime conviction »?) plus que d'un strict raisonnement juridique. Mais après tout le droit n'est pas une science exacte et le fait de tenir compte de la contribution de l'investisseur à la survenance de son préjudice constitue en soi un important acquis.

\section{Dommage moral}

La question du dommage moral a fait son apparition dans le contentieux transnational voilà quelques années, mais les succès des investisseurs en la matière ont

233. CPA (CNUDCI), Copper Mesa Mining Corporation c. Équateur, n 201-2, sentence, 15 mars 2016. 234. Voir aussi infra, III, A, 3.

235. Voir l'opinion dissidente de l'arbitre B. Cremades dans l'affaire CIRDI Fraport AG Frankfurt Airport Services Worldwide c. Philippines, $\mathrm{n}^{\circ} \mathrm{ARB} / 03 / 25$, sentence, 16 août 2007, $\S 37$ et s.

236. Chemin de fer de la baie de Delagoa (Royaume-Uni et États-Unis c. Portugal), reproduit in Henri

LA FonTAine, Pasicrisie internationale 1794-1900, La Haye, Nijhoff, p. 397 (p. 402 ).

237. Copper Mesa Mining Corporation c. Équateur, § 6.102. 
été plutôt mitigés ${ }^{238}$. Globalement, la jurisprudence de l'année 2016 n'inverse pas la tendance mais cela n'empêche nullement les requérants de tenter leur chance de plus en plus fréquemment. Les tribunaux sont toutefois nombreux à insister sur le fait que le standard de preuve est particulièrement élevé en la matière, ce qui explique au reste que les demandes en ce sens soient généralement rejetées. Comme tout dommage, le dommage moral suppose d'abord que sa preuve soit établie, en l'absence de quoi la réparation doit bien entendu être écartée ${ }^{239}$. Mais quand bien même des preuves existeraient, elles devraient établir l'existence d'une conduite atteignant un degré de gravité tel que l'indemnisation du dommage moral pourrait se justifier ${ }^{240}$. Il semble d'ailleurs qu'en la matière, le raisonnement des tribunaux soit davantage porté sur le comportement de l'État que sur le dommage lui-même. Ainsi dans l'affaire Oxus, le motif du refus d'indemniser le dommage moral est clair : "the Arbitral Tribunal has not received enough evidence to support a conclusion that the prosecutions initiated were not justified in the circumstances or were of such an egregious nature as to constitute a breach of Uzbek or international $l a w »{ }^{241}$. On peut s'étonner d'un tel glissement du raisonnement, car à la limite, la gravité du comportement de l'État peut certes avoir une incidence sur l'importance du dommage, mais pas toujours sur sa nature. Savoir si une indemnisation doit être prononcée suppose d'abord d'identifier si un préjudice moral a été subi ce qui, dans le cas d'un investissement, s'apparenterait très probablement à une atteinte à l'image ou à la réputation et, plus rarement, à une souffrance psychologique si des individus ont été directement affectés par les mesures étatiques (emprisonnement, harcèlement, etc.). Dans cette affaire en tout cas, ce n'est pas le point de vue qui fut adopté par le tribunal et son raisonnement s'en trouve assez affaibli. Il ne faudrait pas que le dommage moral soit utilisé par les tribunaux comme une manière dissimulée d'imposer des dommages-intérêts punitifs même si c'est parfois le sentiment qui transparait à la lecture d'une partie de la jurisprudence.

De façon plus originale, la question du dommage moral a été évoquée cette année dans le cadre d'une demande reconventionnelle ${ }^{242}$ : c'est donc l'État qui estimait en avoir été victime du fait des agissements de l'investisseur. Le tribunal ne s'est pas opposé à cette possibilité sur le principe, tout en rappelant que l'indemnisation du dommage moral était particulièrement rare dans le contentieux arbitral de l'investissement ${ }^{243}$. Il admet en outre qu'un État pourrait subir un dommage d'une telle nature s'il était poursuivi par un investisseur peu scrupuleux enclenchant une procédure sans aucun motif valable et de manière abusive car ce type d'agissement pourrait affecter l'image du pays et diminuer son attractivité auprès des autres investisseurs internationaux. En l'occurrence, toutefois, le tribunal s'est refusé à prononcer une telle réparation, estimant que la demande n'était ni fautive ni abusive et qu'elle reposait sur un droit reconnu par la convention de Washington ${ }^{244}$. On peut, cependant, s'interroger sur la bienveillance affichée par le tribunal au sujet de cette possibilité : il n'est pas certain en effet qu'une procédure arbitrale qui affecterait l'image du pays et réduirait son attractivité puisse être considérée véritablement comme un dommage de nature morale. Tout porte à croire au contraire que le risque de diminution de l'attractivité du territoire, quand

238. Voir cette chronique, cet Annuaire, 2015, p. 886.

239. CIRDI, Victor Pey Casado et President Allende Foundation c. Chili, n ${ }^{\circ} \mathrm{ARB} / 98 / 2$, sentence, 13 septembre $2016, \S 243$.

240. Trib. ad hoc (CNUDCI), Oxus c. Ouzbékistan, §§ 895-905.

241. Ibid., §903. Voir également le $\S 904$ sur l'absence de preuve que ces poursuites seraient injustifiées au regard du droit international ou du TBI.

242. Voir infra, III, C, 1.

243. CIRDI, Lundin Tunisia BV c. Tunisie, $\mathrm{n}^{\circ} \mathrm{ARB} / 12 / 30$, sentence, 22 décembre 2015, $§ 374$.

244. Ibid., § 379 . 
bien même il ne se réaliserait pas en pratique, relève du dommage économique puisque c'est bien en termes monétaires qu'il est susceptible de se traduire. L'image d'un pays n'a d'image que le nom : s'il y est porté atteinte, c'est bien en termes de diminution de l'activité économique - investissement étranger, commerce avec les autres États et même flux touristiques - que cette atteinte se manifestera. On peut donc s'interroger sur la possibilité d'envisager un préjudice «purement » moral qui serait subi par un État. À l'extrême, une atteinte à la réputation serait sans doute plus facile à indemniser en tant que préjudice économique, car il est évident qu'une telle atteinte peut se produire en pratique du fait d'un comportement abusif et qu'elle pourrait avoir des conséquences importantes pour l'économie d'un pays. Le recours à une notion aussi délicate à manier que le préjudice moral n'est peut-être donc pas des plus souhaitables.

\section{Standard de réparation}

Les principes présidant à la réparation en droit international ne sont plus contestés par aucun tribunal arbitral. La priorité donnée à la restitution en droit coutumier est néanmoins régulièrement écartée en cas d'impossibilité d'exécution même si rares sont les tribunaux à prendre le temps de vérifier celle-ci, la tendance ultra-majoritaire étant de passer directement au stade de l'indemnisation par équivalent. Il n'en reste pas moins vrai que certains tribunaux demeurent attachés à une stricte orthodoxie et n'envisagent cette dernière qu'en cas d'impossibilité de mettre en œuvre la restitution ${ }^{245}$. En pratique, la restitution se trouve presque systématiquement écartée, de sorte que l'essentiel du contentieux se concentre sur la détermination de l'indemnisation, qui soulève des problématiques complexes.

Parmi elles, le sort à réserver aux dispositions des traités précisant les éléments à prendre en compte pour la compensation d'une expropriation licite dans l'évaluation de la réparation d'un acte illicite demeure incertain. Même si en toute rigueur de telles dispositions ne sont pas applicables, elles peuvent fournir des indications précieuses. Certains tribunaux préfèrent toutefois s'en tenir à la rigueur et les écarter purement et simplement dès lors qu'il est question de tirer les conséquences d'un acte illicite ${ }^{246}$. Mais il se peut aussi que la pertinence de la distinction entre la compensation de l'expropriation licite et l'indemnisation de l'expropriation illicite soit ouvertement écartée dès lors que les parties sont d'accord pour que la date de l'évaluation soit celle de la dépossession et non celle de la sentence ${ }^{247}$. Il est vrai qu'à ce moment précis, la valeur de la propriété est la même que l'expropriation soit licite ou non. La date de l'évaluation est au fond l'élément essentiel dans la distinction entre l'expropriation licite et illicite et on devrait pouvoir considérer d'une manière générale, sous réserve de toute circonstance particulière, qu'une expropriation licite doit être évaluée à la date de l'expropriation alors qu'une expropriation illicite devrait l'être à la date de la sentence ${ }^{248}$. Cette dernière solution

245. CIRDI, Joseph Houben c. Burundi, $\mathrm{n}^{\circ} \mathrm{ARB} / 13 / 7$, sentence, 12 janvier 2016, § 235. Egalement CIRDI, Crystallex International Corporation c. Venezuela, $\mathrm{n}^{\circ} \mathrm{ARB}(\mathrm{AF}) / 11 / 2$, sentence, 4 avril 2016, $\S 724$.

246. CPA (CNUDCI), Murphy Exploration \& Production Company - International c. Équateur, sentence finale partielle, 6 mai 2016, $\$ 422$. Également, appliquant ce principe à la détermination des intérêts : CIRDI, Tenaris SA et Talta-Trading e Marketing Sociedade Unipessoal Lda. c. Venezuela, $\mathrm{n}^{\circ} \mathrm{ARB} / 12 / 23$, sentence, 29 janvier 2016, $\$ 585$.

247. CIRDI, Crystallex International Corporation c. Venezuela, $\mathrm{n}^{\circ} \mathrm{ARB}(\mathrm{AF}) / 11 / 2$, sentence, 4 avril 2016, $\S 843-844$.

248. Cette distinction entre les deux expropriations (licite et illicite) autour de la date de l'évaluation avait été assez clairement exposée dans la sentence ConocoPhillips Petrozuata BV et al. c. Venezuela, $\mathrm{n}^{\circ} \mathrm{ARB} / 07 / 30$, décision sur la compétence et le fond, 3 septembre 2013 , $\$ 342$. Voir à ce sujet cette chronique, cet Annuaire 2013, p. 465. 
semble en tout cas applicable à l'hypothèse générale de tout acte internationalement illicite ${ }^{249}$.

Aucun doute n'est plus en revanche possible quant à l'applicabilité du standard coutumier de la réparation intégrale établi par l'arrêt Chorzów et repris en substance par les Articles de la CDI ${ }^{250}$. En vertu de ce principe, l'État responsable est donc tenu d'indemniser à la fois le damnum emergens et le lucrum cessans ${ }^{251}$. Cette dernière affirmation soulève cependant la question de l'indemnisation du profit futur, qui n'est jamais aisée. Si les tribunaux semblent l'admettre sur le principe, ils ne l'appliquent effectivement que dans le cas où la perspective du profit est établie avec un degré suffisant de certitude car le tribunal est alors tenu de reconstruire le profit qui aurait été dégagé par l'investissement sans l'acte illicite ${ }^{252}$. Cela peut être le cas même dans l'hypothèse où l'investisseur n'a pas eu la possibilité de démarrer effectivement son activité mais qu'il est suffisamment certain que, dans le cas contraire, il eût dégagé un profit dès le départ ${ }^{253}$. Il importe alors, bien entendu, d'établir avec fermeté que la perte de perspective de profit est directement liée aux actes de l'État, ce qui ramène la question à un problème de causalité ${ }^{254}$. La question de la perte de chance s'inscrit également dans la même logique, quoiqu'elle soit légèrement différente. Mais le principe même de son indemnisation est admis, pour autant là aussi qu'un lien de causalité avec les actes illicites soit établi ${ }^{255}$. En outre, une certaine méfiance vis-à-vis du caractère insaisissable de la notion peut être perçue dans une partie de la jurisprudence si bien que la perte de chance n'est sans doute pas le motif de réparation le plus favorable pour les investisseurs ${ }^{256}$. Dans cette optique, d'ailleurs, certains tribunaux ont pu refuser d'appliquer la méthode du discounted cash flow, reposant précisément sur des calculs prospectifs du développement à venir de l'entreprise, dans des hypothèses particulières : ce fut notamment le cas dans l'affaire Windstream Energy, le tribunal ayant jugé que le projet en était à un stade trop précoce de développement pour que la perspective de dégagement d'un profit soit établie avec une suffisante certitude ${ }^{257}$.

Cette dernière remarque soulève d'ailleurs la question, particulièrement épineuse, des méthodes à mettre en œuvre pour conduire l'opération d'évaluation proprement dite. Le principe de la réparation intégrale est simple dans son affirmation mais il l'est beaucoup moins dans sa mise en œuvre. Plusieurs tribunaux ont d'ailleurs pris les devants des critiques en admettant ouvertement que l'évaluation du préjudice n'était pas une science exacte, sous-entendant qu'une part de subjectivité ne pouvait être totalement mise à l'écart ${ }^{258}$. Il est vrai que de ce

249. CPA, Windstream Energy LLC c. Canada, $\mathrm{n}^{\circ}$ 2013-22, sentence, 27 septembre 2016, $\$ 484$.

250. Murphy Exploration \& Production Company - International c. Équateur, sentence finale partielle, 6 mai 2016, $\S$ 423-424. Dans le même sens : CIRDI, Vestey Group Limited c. Venezuela, n ARB/06/4, sentence, 15 avril 2016, § 333 ; CIRDI, Joseph Houben c. Burundi, n ARB/13/7, sentence, 12 janvier 2016, $\$ \$ 218$ et s. ; CIRDI, Crystallex International Corporation c. Venezuela, $\mathrm{n}^{\circ} \mathrm{ARB}(\mathrm{AF}) / 11 / 2$, sentence, 4 avril 2016, $\$ \$ 846$ et s. ; CIRDI, Victor Pey Casado et President Allende Foundation c. Chili, n ${ }^{\circ}$ ARB/98/2, sentence, 13 septembre 2016 , $\S \S 201$ et s.

251. CPA (CNUDCI), Flemingo Dutyfree Shop Private Ltd c. Pologne, sentence, 12 août 2016, § 865. 252. Ibid., § 874 .

253. CIRDI, Crystallex International Corporation c. Venezuela, § 877.

254. CPA (CNUDCI), Murphy Exploration \& Production Company - International c. Équateur, sentence finale partielle, 6 mai 2016, $\S \S 466-467$.

255. Voir CPA (CNUDCI), Flemingo Dutyfree Shop Private Ltd c. Pologne, sentence, 12 août 2016, $\S \$ 910-931$, écartant finalement cette indemnisation pour défaut de causalité.

256. CPA (CNUDCI), Copper Mesa Mining Corporation c. Équateur, n 201-2, sentence, 15 mars $2016, \S 7.24$.

257. CPA, Windstream Energy LLC c. Canada, n 2013-22, sentence, 27 septembre 2016, $§ 475$.

258. CPA (CNUDCI), Copper Mesa Mining Corporation c. Équateur, n 201-2, sentence, 15 mars 2016, $§ 7.24$; CIRDI, Crystallex International Corporation c. Venezuela, $\mathrm{n}^{\circ} \mathrm{ARB}(\mathrm{AF}) / 11 / 2$, sentence, 4 avril $2016, \S 886$. 
point de vue, la jurisprudence peut encore paraître assez fluctuante, malgré des efforts déployés par les arbitres pour faire reposer le raisonnement - ou au moins donner cette impression - sur des bases objectives. L'exemple de la « juste valeur de marché » (fair market value) est assez révélateur à cet égard : si certains traités la mentionnent comme valeur de référence (mais généralement dans le cadre des clauses d'expropriation, donc pour qu'elle s'applique aux expropriations licites), plusieurs tribunaux ont admis qu'il s'agissait là de la meilleure manière d'assurer la réparation intégrale du préjudice conformément aux principes du droit international ${ }^{259}$. Dans certaines affaires, les parties avaient même exprimé leur accord à cet égard, ne laissant donc la place à aucun doute ${ }^{260}$. On peut considérer que cette valeur correspond à celle qu'un acheteur serait prêt à accepter pour acquérir l'investissement dans le contexte d'un marché libre ${ }^{261}$. Mais cette méthode n'est pas la seule applicable pour parvenir à effacer toutes les conséquences de l'illicite en application du dictum de Chorzów ${ }^{262}$. Pour déterminer quelle méthode est appropriée, il convient donc de prendre en compte la rédaction du traité applicable - en prenant garde à ne pas appliquer telle quelle la disposition relative à l'expropriation licite - et l'accord des parties ${ }^{263}$. En tout état de cause, certains tribunaux n'hésitent pas à appliquer plusieurs méthodes et, en cas de résultats différents, à calculer leur moyenne pour parvenir au montant de l'indemnisation ${ }^{264}$.

Ce ne sont là cependant que des considérations d'ordre très général, qui ne permettent pas d'éviter dans bien des cas le recours à l'expertise financière. Il semble tout de même que les tribunaux tentent de tenir compte de paramètres d'ensemble afin de ne pas appliquer aveuglément et de manière trop automatique des expertises pas toujours franchement compréhensibles. A contrario, d'ailleurs, un tribunal qui rejetterait des expertises en la matière sans apporter de justification à sa position encourrait l'annulation pour défaut de motifs ${ }^{265}$. En outre, plusieurs éléments viennent offrir aux arbitres des variables d'ajustement sur les chiffres qui leur sont ainsi soumis. Il importe ainsi de rappeler que l'indemnisation en droit international vise à réparer le dommage mais n'est assortie d'aucune dimension punitive, ce qui peut avoir une incidence sur le montant ${ }^{266}$. Dans le contexte particulier de l'expropriation, un tribunal a eu également l'occasion d'apporter une précision importante sur la situation à rétablir en vertu du droit international de la responsabilité : cette situation correspond à celle qui aurait existé s'il y avait eu indemnisation et non celle de l'investisseur demeuré propriétaire. L'État conserve en effet, en tout état de cause, son droit d'exproprier de sorte qu'un investisseur n'est jamais garanti de conserver sa propriété ${ }^{267}$. En matière d'évaluation des biens d'ailleurs, il est entendu que tous les paramètres doivent être pris en compte, dont notamment les contestations qui pourraient affecter le droit de propriété du

259. CIRDI, Crystallex International Corporation c. Venezuela, $\mathrm{n}^{\circ} \mathrm{ARB}(\mathrm{AF}) / 11 / 2$, sentence, 4 avril 2016, § 853 ; CPA (CNUDCI), Murphy Exploration \& Production Company - International c. Équateur, sentence finale partielle, 6 mai 2016, § 482.

260. CIRDI, Rusoro Mining Ltd c. Venezuela, $\mathrm{n}^{\circ} \mathrm{ARB}(\mathrm{AF}) / 12 / 15$, sentence, 22 août 2016, § 647.

261. CIRDI, Tenaris SA et Talta - Trading e Marketing Sociedade Unipessoal Lda. c. Venezuela, $\mathrm{n}^{\circ} \mathrm{ARB} / 12 / 23$, sentence, 29 janvier 2016, $\$ 519$.

262. Comité ad hoc CIRDI, Adem Dogan c. Turkménistan, n ARB/09/9, décision sur l'annulation, 15 janvier 2016, § 164.

263. Dans l'affaire Adem Dogan, ibid., c'est bien le défaut d'accord des parties qui avait conduit le tribunal à écarter le recours à cette méthode sans que le Comité ad hoc trouve à y redire.

264. CIRDI, Crystallex International Corporation c. Venezuela, $\mathrm{n}^{\circ} \mathrm{ARB}(\mathrm{AF}) / 11 / 2$, sentence, 4 avril $2016, \S 916$.

265. Comité ad hoc CIRDI, TECO Guatemala Holdings LLC c. Guatemala, n ARB/10/23, décision sur l'annulation, 5 avril 2016 , $\S 127$ et s.

266. CIRDI, Saint-Gobain Performance Plastics Europe c. Venezuela, n ARB/12/13, décision sur la responsabilité et les principes de la réparation, 30 décembre 2016, $§ 719$. 267. Ibid., § 873 . 
requérant, qui sont de nature à avoir un effet défavorable sur la valeur de ses possessions ${ }^{268}$. Il en ressort que l'évaluation n'est certes pas une science exacte mais que la jurisprudence de plus en plus développée à son sujet participe tout de même à la construction d'un cadre global. Les arbitres continuent certes de bénéficier d'une marge de liberté au sein de ce cadre, mais cela semble indispensable pour leur garantir la possibilité de s'adapter à la diversité des situations factuelles qui leur sont soumises.

\section{III. - ARBITRAGE TRANSNATIONAL ET DROIT DU CONTENTIEUX INTERNATIONAL}

La procéduralisation du contentieux arbitral transnational ne se dément pas. Certes, d'épineuses questions liées aux procédures multipartites ont été repoussées du fait de l'accord survenu entre l'Argentine et les petits porteurs qui la mettaient en cause dans le cadre de la restructuration de sa dette ${ }^{269}$. Mais plusieurs questions importantes ont encore été abordées en 2016, portant sur la compétence des tribunaux et la recevabilité des demandes (A), le droit applicable (B), les procédures incidentes $(\mathrm{C})$ ou encore le caractère définitif des décisions et sentences (D). Les décisions rendues apportent alors un éclairage sur le droit du contentieux international mais également sur des enjeux plus larges, qui viennent se nicher dans les méandres de la procédure arbitrale. Ainsi de la personnalité juridique internationale des investisseurs, dont certaines décisions ont confirmé qu'ils disposaient bien de droits ${ }^{270}$ quand d'autres ont souligné qu'ils pouvaient aussi être soumis à des obligations en vertu du droit international ${ }^{271}$.

\section{A. Compétence et recevabilité}

\section{Compétence ou recevabilité?}

La distinction entre compétence et recevabilité reste mal assurée dans le contentieux arbitral transnational, aussi bien dans son principe que dans ses modalités. Arbitres et doctrine demeurent ainsi divisés sur la qualification qu'il convient de réserver aux conditions procédurales préalables à la saisine du tribunal, telles que les exigences de saisine des juridictions internes, de notification du différend ou de négociation ${ }^{272}$. Bien que les conséquences de cette classification demeurent mal établies ${ }^{273}$ et alors même que les parties s'accordaient pour voir dans la saisine des juges internes une condition mise à la compétence du tribunal, le tribunal de l'affaire Içkale l'a analysée comme une condition de recevabilité de la demande. Pour les arbitres majoritaires, les limites ratione personae, materiae et temporis mises au consentement à l'arbitrage fixent la compétence du tribunal, tandis que les

268. CIRDI, Joseph Houben c. Burundi, $\mathrm{n}^{\circ} \mathrm{ARB} / 13 / 7$, sentence, 12 janvier 2016, $\S 241$ et $\mathrm{s}$.

269. CIRDI, Abaclat et al. c. Argentine, $\mathrm{n}^{\circ} \mathrm{ARB} / 07 / 5$, sentence sur le règlement amiable, 29 décembre 2016.

270. CIRDI, MNSS BV et Recupero Credito Acciaio NV c. Monténégro, ARB(AF)/12/8, sentence, 4 mai 2016, §§ 148-165; voir infra, B, 1.

271. CIRDI, Urbaser SA et Consorcio de Aguas Bilbao Bizkaia, Bilbao Biskaia Ur Partzuergoa $c$. Argentine, $\mathrm{n}^{\circ} \mathrm{ARB} / 07 / 26$, sentence, 8 décembre 2016, $\S \S 1143$ et s. ; voir infra $\mathrm{C}, 1$.

272. Voir cette chronique, cet Annuaire, 2014, pp. 593-596.

273. Envisageant les conséquences possibles, voir Michael WAIBEL, « Investment Arbitration : Jurisdiction and Admissibility ", in Mark GunEnberG et al. (dir.), International Investment Law, Londres, Hart, 2015, pp. 1212 et s. 
exigences procédurales que doivent respecter les investisseurs avant d'introduire leur demande en conditionnent la recevabilité. La distinction ne serait donc pas subjective, fonction de l'intention exprimée par des États libres de conditionner leur consentement, mais objective, selon que c'est le type de différend dont le tribunal peut connaître ou les conditions procédurales de sa saisine qui sont en cause ${ }^{274}$.

Ce raisonnement peut convaincre et se retrouve d'ailleurs peu ou prou dans d'autres décisions. Ainsi, tandis qu'ici comme ailleurs ${ }^{275}$ l'existence d'un différend semble pouvoir être considérée comme une condition de la compétence du tribunal ${ }^{276}$, l'exigence d'une notification préalable de ce différend ressortirait à la recevabilité de la demande ${ }^{277}$. Le raisonnement de la majorité dans l'affaire Içkale suscite toutefois des réserves en ce qu'il s'écarte de plusieurs précédents sans véritablement s'en justifier et, surtout, en ce que la classification de la saisine préalable des juridictions internes comme relevant de la recevabilité annonce une appréciation particulièrement souple du respect de cette condition ${ }^{278}$. Peu importe pour la majorité du tribunal que le demandeur n'ait jamais saisi les juridictions turkmènes avant de s'adresser au CIRDI dès lors qu'elles ont pu connaître d'une partie du différend à l'initiative des autorités locales. Cela n'est pas dit, mais l'on comprend à la lecture de cette sentence que, tandis que le respect des conditions de compétence du tribunal doit être rigoureusement analysé, celui des exigences qui tiennent à la recevabilité des demandes peut faire l'objet d'une appréciation souple. L'opposition entre les arbitres sur la question est donc essentiellement conditionnée par la rigueur avec laquelle ils entendent apprécier le respect d'une condition ${ }^{279}$. Pourtant, dans l'un et l'autre cas, le résultat est le même : qu'il soit incompétent ou que la demande soit irrecevable, le tribunal ne peut se prononcer au fond, même si l'irrecevabilité peut, dans certains cas, être corrigée... Dans certains cas, mais pas dans tous. Ainsi, dans trois affaires les demandeurs se sont heurtés en tout ou partie aux délais de prescription posés par les traités pertinents, sans que la classification de cette condition comme relevant de la compétence ou de la recevabilité ne change quoi que ce soit à l'affaire ${ }^{280}$.

\section{Appréciation du consentement de l'État}

Le consentement des États à l'arbitrage résulte aujourd'hui le plus souvent des traités de promotion et de protection des investissements qu'ils concluent. Cet élargissement désormais classique de la possibilité de recourir à l'arbitrage par consentement dissocié ne semble pas suffire à certains demandeurs qui n'hésitent

274. CIRDI, İçkale İş̧aat Limited Şirketi c. Turkménistan, n ARB/10/24, sentence, 8 mars 2016 , $\S \S 234-247$.

275. CIJ, Obligations relatives à des négociations concernant la cessation de la course aux armes nucléaires et le désarmement nucléaire (Îles Marshall c. Royaume-Uni), exceptions préliminaires, arrêt du 5 octobre 2016, Rec. CIJ, 2016, §36. Voir toutefois Carlo SANTULLI, Droit du contentieux international, Paris, LGDJ, 2015, $2^{\mathrm{e}}$ éd., pp. 225 et s., qui classe l'existence d'un différend parmi les conditions de recevabilité.

276. CIRDI, Crystallex International Corporation c. Venezuela, $\mathrm{n}^{\circ} \mathrm{ARB}(\mathrm{AF}) / 11 / 2$, sentence, 4 avril $2016, \S \S 445-458$, où le tribunal reformule l'exception tenant à l'absence de notification et de négociation préalable comme une exception portant sur l'existence et la teneur d'un différend.

277. CIRDI, RREEF Infrastructure (GP) Limited et RREEF Pan-European Infrastructure Two Lux SARL c. Espagne, n ARB/13/30, décision sur la compétence, 6 juin 2016, § 225 ; CIRDI, Joseph Houben c. Burundi, $\mathrm{n}^{\circ} \mathrm{ARB} / 13 / 7$, sentence, 12 janvier 2016, $\S 137-148$.

278. Sentence précitée, $\S 261$.

279. Voir déjà cette chronique, cet Annuaire, 2011, pp. 568-572.

280. CIRDI, Corona Materials, LLC c. République dominicaine, ARB(AF)/14/3, sentence sur l'exception préliminaire, 31 mai 2016, $\$ \S 181-238$; CIRDI, Rusoro Mining Ltd c. Venezuela, $\mathrm{n}^{\circ} \mathrm{ARB}(\mathrm{AF}) / 12 / 15$, sentence, 22 août 2016, $\S \S 203-240$; CIRDI, Spence International Investments, LLC et al. c. Costa Rica, $\mathrm{n}^{\circ} \mathrm{UNCT} / 13 / 2$, sentence intérimaire, 25 octobre $2016, \S 270$. 
pas à rechercher dans les méandres des clauses de la nation la plus favorisée des extensions du consentement étatique. Cet activisme a suscité des réactions de la part d'États qui, tels le Venezuela, ont dénoncé la convention de Washington créant le CIRDI, décision dont les conséquences ont été appréhendées par les arbitres en 2016.

\section{a) Consentement et clause de la nation la plus favorisée}

Depuis la décision Maffezini ${ }^{281}$, les tribunaux ont été si régulièrement confrontés à l'argument selon lequel le consentement à l'arbitrage donné par l'État d'accueil de l'investissement pourrait être élargi, voire apparaître, par l'effet d'une clause de la nation la plus favorisée insérée dans le traité invoqué que la Commission du droit international a consacré à cette question l'essentiel du rapport relatif à ladite clause ${ }^{282}$. Ce rapport a d'ailleurs rapidement trouvé un écho dans la pratique arbitrale, sans pour autant permettre de réconcilier les antagonismes. L'affaire Venezuela US l'illustre, dans laquelle les demandeurs cherchaient, à partir de la clause de la nation la plus favorisée du traité Barbade/Venezuela, un substitut au consentement à l'arbitrage remis en cause par la dénonciation par l'Etat défendeur de la convention de Washington ${ }^{283}$. L'affaire était d'autant plus complexe qu'à l'instar du modèle britannique, mis en œuvre notamment dans l'affaire Garanti Koza, ce traité prévoyait expressément que la clause de la nation la plus favorisée qu'il contient devait s'appliquer à certaines dispositions, dont celle relative au règlement des différends. Et, comme dans l'affaire Garanti Koza ${ }^{284}$, les arbitres se sont séparés en une majorité, pour qui cette formulation doit conduire à considérer que les parties au traité ont admis que les conditions posées au consentement à l'arbitrage puissent être écartées au profit de celles, plus favorables, d'un autre traité, et une minorité, qui se trouve être dans les deux cas spécialiste de droit international public (en plus d'être universitaire et genevois), pour qui il s'agit là de rien moins que d'importer le consentement de l'État à l'arbitrage depuis un autre traité alors que celui exprimé dans le traité initial a disparu 285.

L'opposition porte en effet pour l'essentiel sur cette question de la distinction entre le consentement à l'arbitrage proprement dit et les modalités de l'arbitrage. Pour les arbitres majoritaires, le consentement à l'arbitrage du Venezuela n'a pas été remis en cause mais ce sont les modalités prévues par le traité (à savoir l'arbitrage CIRDI) qui ne peuvent plus être mises en œuvre du fait de la dénonciation de la convention de Washington et qui peuvent alors être remplacées par celles, plus favorables, prévues par un autre traité (l'arbitrage ad hoc suivant le règlement CNUDCI). Pour l'arbitre dissident au contraire, non seulement la clause de la nation la plus favorisée du traité pertinent n'est pas de celles qui couvrent le règlement des différends bien que son champ d'application soit maladroitement formulé, mais elle ne saurait quoi qu'il en soit permettre de réactiver un consentement à l'arbitrage disparu après la dénonciation de la convention de Washington ${ }^{286}$. Bien qu'ils se séparent sur la question ici décisive du maintien ou de la disparition du consentement à l'arbitrage dans le traité initial, les arbitres s'accordent toutefois

281. CIRDI, Maffezini c. Espagne, n ARB/97/7, décision sur la compétence, 25 janvier 2000.

282. Commission du droit international, Groupe d'étude sur la clause de la nation la plus favorisée,

Rapport final, in Rapport de la CDI sur les travaux de sa 67e session, A/70/10 (2015), Annexe, pp. 158-207. 283. Voir infra.

284. CIRDI, Garanti Koza LLP c. Turkménistan, n ARB/11/20, décision sur la compétence, 3 juillet 2013 et op. diss. Laurence Boisson de Chazournes.

285. CPA (CNUDCI), Venezuela US, SRL c. Venezuela, $\mathrm{n}^{\circ} 2013-34$, sentence partielle sur la compétence, 26 juillet 2016, $\S 100-130$.

286. Op. diss. Marcelo Kohen. 
sur un point de principe essentiel : une clause de la nation la plus favorisée ne peut permettre d'importer ce consentement depuis un autre traité. La décision s'inscrit donc dans « la tendance des tribunaux arbitraux à se défendre de fonder le consentement [sur la clause de la nation la plus favorisée] même lorsqu'ils acceptent de [lui] donner une portée procédurale ${ }^{287}$. Des raisons de deux ordres peuvent être mises en évidence pour l'expliquer, à partir de l'opinion dissidente de M. Kohen. Sur le plan technique, à supposer que la clause de la nation la plus favorisée fasse peser sur l'État l'obligation de consentir à l'arbitrage, elle n'emporte pas par elle-même consentement et un tribunal arbitral ne saurait procéder à une forme d'exécution forcée de cette obligation. Sur un plan plus large, " the way to promote the acceptance of, recourse to and reliance on international investment arbitration is not by imposing it without the fundamental requirement of State consent. On the contrary, this would only serve to undermine its credibility 288.

Pareil avertissement ne paraît pas vain. En effet, les tribunaux sont confrontés à des demandes sans cesse plus audacieuses d'investisseurs qui croient trouver dans l'arbitrage transnational leur salut. Ainsi des requérants de l'affaire Menzies. Aucun traité d'investissement n'ayant été conclu entre leur État de nationalité et leur État d'accueil, ceux-ci entendaient s'appuyer pour soumettre leur différend à un tribunal arbitral sur la clause de la nation la plus favorisée contenue dans... l'Accord général sur le commerce des services (AGCS) de l'Organisation mondiale du commerce. Autrement dit, les États parties à l'AGCS qui consentiraient à l'arbitrage pour le règlement des différends les opposant aux investisseurs d'un État quelconque verraient leur consentement étendu aux différends les opposant aux prestataires de service de l'ensemble des États parties à l'AGCS par l'effet de la clause de la nation la plus favorisée figurant dans ce texte. Pour le dire avec les mots du tribunal de cette affaire, il lui était demandé de " 'composer' un consentement de la Défenderesse en 'collant' des pièces disparates à la suite d'une analyse d'intégration [...] entre la clause NPF et les offres d'arbitrage adressées à des investisseurs d'États tiers » 289 . Fort heureusement, les arbitres ne se sont pas prêtés à semblable composition en retenant que, outre que la clause en question est muette sur l'arbitrage et que son interprétation, notamment à la lumière des travaux préparatoires, est bien trop délicate pour pouvoir y lire un consentement clair, exprès et non équivoque, son éventuelle violation par l'Etat concédant pourrait seulement être contestée par l'État bénéficiaire devant l'Organe de règlement des différends de l'OMC ${ }^{290}$. La Commission du droit international posait d'ailleurs le problème en ces termes interétatiques en s'interrogeant, sans apporter de réponse, sur le point de savoir « si un Membre de l'OMC pourrait, en vertu de l'article II de l'AGCS, demander à bénéficier des dispositions d'un traité bilatéral d'investissement conclu par un autre Membre de l'OMC avec un État tiers lorsque les mesures que contient ledit traité confèrent un traitement plus favorable aux prestataires de services de l'État tiers en question " ${ }^{291}$.

Au-delà de cette question, qui confine à l'interprétation du principe ejusdem generis, les arbitres insistent, comme l'arbitre dissident de l'affaire Venezuela US, sur la différence entre l'existence (éventuelle) de l'obligation d'offrir l'accès à l'arbitrage aux fournisseurs de service de l'ensemble des États parties à l'AGCS au titre de la clause de la nation la plus favorisée et sa mise en œuvre, qui doit être le fait de l’État débiteur, non du tribunal. En effet, souligne le tribunal, « les États

287. Claire CRÉPet DAigremont, La clause de la nation la plus favorisée, Paris, Pedone, 2015, p. 395. 288. Op. diss. Marcelo Kohen, § 59. Contra, Claire CREPET DAIGREMONT, op. cit., pp. 396-397. 289. CIRDI, Menzies Middle East and Africa SA et Aviation Handling Services International Ltd c. Sénégal, $\mathrm{n}^{\circ} \mathrm{ARB} / 15 / 21$, sentence, 5 août $2016, \S 135$.

290. Ibid., §§ 129-151.

291. Rapport précité, p. $12, \S 51$. 
gardent toujours la faculté de refuser de respecter une obligation, qui reste une prérogative inéluctable de leur souveraineté » ${ }^{292}$, quitte alors à mettre en jeu leur responsabilité. Appliqué à la lettre, ce raisonnement conduit à écarter l'application de la clause de la nation la plus favorisée aux dispositions relatives au règlement des différends : tant que la compétence d'un tribunal n'a pas été établie, un investisseur ne saurait se plaindre devant lui de la violation à son endroit de la clause de la nation la plus favorisée ${ }^{293}$.

\section{b) Consentement et dénonciation de la convention de Washington}

Les surprises de l'arbitrage autant que des évolutions politiques internes ont pu conduire certains États à remettre en cause leur participation au système. Tel fut le cas du Venezuela, qui fit le choix en 2012 de dénoncer la convention de Washington créant le CIRDI après avoir fait l'objet de plusieurs condamnations. $\mathrm{Ni}$ la possibilité de dénoncer cette convention, ni la date de ses effets ne posaient de difficultés : à compter du 24 juillet 2012 , soit six mois après la réception par le secrétariat de sa notification en ce sens, le Venezuela avait cessé d'être partie à la convention. Mais les conséquences de cette dénonciation sur le consentement de cet État à l'arbitrage, exprimé le plus souvent par traité, continuaient de faire débat ${ }^{294}$. De ce point de vue, les décisions rendues en 2016 apportent de sérieux tempéraments à la dénonciation vénézuélienne.

Le tribunal de l'affaire Venezuela US a admis que cette dénonciation avait pour effet de priver les demandeurs de la possibilité, pourtant offerte par le traité Barbade/Venezuela toujours en vigueur, de saisir le CIRDI. Et comme ce tribunal a également considéré que l'alternative consistant à saisir un tribunal ad hoc ou à recourir au mécanisme supplémentaire CIRDI n'avait été prévue que tant que le Venezuela n'était pas devenu partie à la convention de Washington, il semblait devoir se déclarer incompétent pour défaut de consentement du défendeur ${ }^{295}$. C'était toutefois sans compter sur l'interprétation par la majorité des arbitres des effets de la clause de la nation la plus favorisée figurant dans ce traité. On l'a dit, pour les arbitres majoritaires, la dénonciation de la convention de Washington n'avait pas fait disparaître le consentement du Venezuela à l'arbitrage mais seulement à l'arbitrage CIRDI. La clause de la nation la plus favorisée insérée dans le traité pertinent permettait donc encore aux demandeurs de se prévaloir des modalités d'arbitrage plus favorables figurant dans d'autres traités conclus par le Venezuela, et donc de saisir un tribunal ad hoc ${ }^{296}$.

D'autres tribunaux se sont quant à eux prononcés sur l'effet dans le temps de la dénonciation. L'article 71 de la convention de Washington prévoit classiquement qu'elle prend effet six mois après sa notification, tandis que l'article 72 précise qu'elle est sans effet sur les droits et obligations découlant d'un consentement antérieur. Certains tribunaux se sont alors déclarés compétents sans difficulté pour connaitre des réclamations introduites entre la notification de la dénonciation et sa date d'effet ${ }^{297}$, alors même d'ailleurs qu'elles n'auraient été enregistrées par le

292. Sentence Menzies, $\S 137$.

293. Voir déjà, l'op. diss. de Laurence Boisson de Chazournes dans l'affaire Garanti Koza, précit.

294. Julien CAZALA, " La dénonciation de la convention de Washington établissant le CIRDI ", cet Annuaire, 2012, pp. 551-565.

295. CPA (CNUDCI), Venezuela US, SRL c. Venezuela, $\mathrm{n}^{\circ} 2013-34$, sentence partielle sur la compétence, 26 juillet $2016, \S \S 68-90$.

296. Ibid., §§ 100-130.

297. CIRDI, Saint-Gobain Performance Plastics Europe c. Venezuela, n ARB/12/13, décision sur la responsabilité et les principes de la réparation, 30 décembre $2016, \S 358$, précisant que la notification du différend est antérieure à celle de la dénonciation. 
secrétariat que plus tardivement ${ }^{298}$. Le tribunal de la deuxième affaire Tenaris a toutefois consacré davantage de développements à la question. Le Venezuela soutenait en effet que l'article 72 de la convention avait pour effet que la rencontre des consentements des parties devait être antérieure à la notification de la dénonciation. Le tribunal ne s'est toutefois pas prononcé sur la question, estimant que, quoi qu'il en soit, la rencontre des consentements ne résultait pas de l'introduction des demandes, survenues entre la date de la dénonciation et sa date d'effet, mais de la notification du différend, antérieure à la date de la dénonciation ${ }^{299}$. La question était particulièrement délicate s'agissant de l'une des demandes, pour laquelle la période de six mois de négociation préalable à la saisine du tribunal venait à peine de débuter à la date de la dénonciation. Mais, tranchant entre des prétentions soutenues respectivement par A. Reinisch et Ch. Schreuer, le tribunal a jugé que l'exigence d'une période de négociation n'empêchait pas l'investisseur de donner $a b$ initio son consentement à l'arbitrage en cas d'échec des discussions. La rencontre des consentements avait donc eu lieu dès la notification du différend et la dénonciation ne pouvait la remettre en cause ${ }^{300}$.

Bref, dans chacune de ces affaires, le Venezuela a échoué à démontrer que sa dénonciation de la convention de Washington remettait en cause son consentement à l'arbitrage. Certes, les solutions retenues traduisent les particularités de chaque espèce, les tribunaux aménageant les effets de la dénonciation sans les remettre radicalement en cause. Ainsi, les décisions rendues ne retiennent pas la théorie de l'offre, selon laquelle la dénonciation de la convention de Washington serait sans effet sur les offres unilatérales d'arbitrage émises auparavant ${ }^{301}$. Mais ces exemples illustrent tout de même la difficulté qu'il peut y avoir à remettre en cause les engagements pris à l'égard des investisseurs.

\section{Prise en compte de l'attitude du demandeur}

Les États mis en cause par des investisseurs étrangers devant les tribunaux arbitraux insistent désormais de manière régulière sur les torts de ces derniers afin de mettre en cause la compétence du tribunal ou la recevabilité de la requête. Deux arguments sont principalement avancés afin de nier au demandeur la protection de l'instrument qu'il invoque : on lui reproche tantôt d'avoir violé le droit local, tantôt de ne pas avoir de lien suffisant avec l'État dont il se dit le national.

\section{a) Conformité au droit local de l'investissement}

Les décisions et sentences qui abordent la question de la conformité au droit local des investissements ou des agissements des investisseurs tendent à se multiplier. Au cours de la période examinée, deux demandeurs se sont d'ailleurs heurtés à cet argument : le tribunal de l'affaire Grupo Francisco Hernando Contreras s'est ainsi déclaré incompétent au motif que le demandeur ne bénéficiait pas d'un investissement protégé, les contrats dont il se prévalait n'ayant pas été conclus conformément aux procédures prévues par le droit administratif guinéen ${ }^{302}$, tandis que celui de l'affaire Churchill Mining a jugé la requête dont il était saisi irrecevable après

298. CIRDI, Rusoro Mining Ltd c. Venezuela, $\mathrm{n}^{\circ} \mathrm{ARB}(\mathrm{AF}) / 12 / 15$, sentence, 22 août 2016, $\S ~ 260-268$, s'agissant de la possibilité d'accéder au mécanisme supplémentaire CIRDI.

299. CIRDI, Tenaris SA et Talta - Trading e Marketing Sociedade Unipessoal Lda. c. Venezuela, $\mathrm{n}^{\circ} \mathrm{ARB} / 12 / 23$, sentence, 12 novembre 2016, $\S 62$.

300. Ibid., §§ 122-144.

301. Pour une présentation, voir Julien CAZALA, op. cit.

302. CIRDI, Grupo Francisco Hernando Contreras SL c. Guinée équatoriale, ${ }^{\circ} \mathrm{ARB}(\mathrm{AF}) / 12 / 2$, sentence sur la compétence, 4 décembre 2015, §§ 199-264. 
que l'examen détaillé des signatures apposées sur les documents pertinents eut révélé que les contrats de concessions minières sur lesquels s'appuyait la demande étaient tout simplement des faux, établis semble-t-il à l'initiative du partenaire local du demandeur ${ }^{303}$. Au-delà de questions factuelles propres à chaque cas particulier et des aspects probatoires ${ }^{304}$, les décisions rendues illustrent la grande diversité des situations envisageables, selon le texte du traité applicable, la gravité des comportements en cause, leur lien avec la demande soumise au tribunal, ou encore le moment de leur réalisation. Trois points peuvent être soulignés à la lecture des décisions et sentences de l'année.

D’abord, la situation diffère désormais assez peu selon que le traité appliqué contient ou non une clause de conformité de l'investissement au droit local. En effet, si les tribunaux peuvent régulièrement s'appuyer sur de telles clauses ${ }^{305}$, ceux qui sont saisis sur la base de textes muets sur la question n'hésitent pas à rechercher, à la suite du tribunal de l'affaire Phoenix Action ${ }^{306}$, dans les principes généraux du droit, dont la bonne foi et l'abus de procédure, le fondement de l'examen de la conformité de l'investissement au droit local ${ }^{307}$ ou à s'appuyer, à l'instar du tribunal de l'affaire World Duty Free ${ }^{308}$, sur l'ordre public international pour dénier toute protection aux investisseurs coupables d'acte de corruption ou de fraude ${ }^{309}$.

Ensuite, les moyens liés aux agissements de l'investisseur doivent être soulevés en temps utile. Certes, la gravité des faits peut justifier un examen tardif du moyen tiré de la corruption ou de la fraude ${ }^{310}$, mais l'État qui n'a jamais mis en cause la conformité de l'investissement au droit local peut se voir opposer son silence. La manière dont celui-ci est pris en compte varie cependant et dépend largement du contexte : tantôt le tribunal estime que le silence gardé par l'État ne saurait s'assimiler à une acceptation de la légalité de l'investissement ${ }^{311}$, tantôt il juge que le fait que le défendeur n'ait jamais mis en cause cette légalité avant le début de la procédure arbitrale l'empêche de soulever cet argument ${ }^{312}$.

Enfin, la manière dont les agissements litigieux des investisseurs sont pris en compte semble devoir dépendre du moment de leur réalisation. Survenus en amont

303. CIRDI, Churchill Mining PLC et Planet Mining Pty Ltd c. Indonésie, $\mathrm{n}^{\circ}$ ARB/12/14 et 12/40, sentence, 6 décembre 2016, $\S 528-532$.

304. CIRDI, Getma International, NCT Necotrans, Getma International Investments, NCT Infrastructure \& Logistique c. Guinée, n ARB/11/29, sentence, 16 août 2016, $\S \S 181-184$, jugeant que l'allégation de corruption n'exige pas de standard de preuve particulier et qu'elle peut être établie sur la base de preuves "claires et convaincantes " permettant de donner au tribunal une « certitude raisonnable ".

305. CIRDI, Grupo Francisco Hernando Contreras SL c. Guinée équatoriale, ${ }^{\circ} \mathrm{ARB}(\mathrm{AF}) / 12 / 2$, sentence sur la compétence, 4 décembre 2015, §§ 199 ; CPA (CNUDCI), Copper Mesa Mining Corporation c. Équateur, $\mathrm{n}^{\circ}$ 201-2, sentence, 15 mars 2016, §§ 5.54-5.60 ; CIRDI, Rusoro Mining Ltd c. Venezuela, $\mathrm{n}^{\circ} \mathrm{ARB}(\mathrm{AF}) / 12 / 15$, sentence, 22 août $2016, \S 289$.

306. CIRDI, Phoenix Action, Ltd. c. République tchèque, n ARB/06/5, sentence, 15 avril 2009.

307. Trib. Ad hoc (CNUDCI), Oxus c. Ouzbékistan, sentence finale, 17 décembre 2015, §§ 706-713; CIRDI, Ampal-American Israel Corp. et al. c. Égypte, $\mathrm{n}^{\circ} \mathrm{ARB} / 12 / 11$, décision sur la compétence, $1^{\text {er }}$ février $2016, \S \S 300-311$. Discutant également la question mais sans la trancher, CIRDI, MNSS BV et Recupero Credito Acciaio NV c. Monténégro, ARB(AF)/12/8, sentence, 4 mai 2016, §§ 209-215 ; CIRDI, Churchill Mining PLC et Planet Mining Pty Ltd c. Indonésie, ${ }^{\circ}$ ARB/12/14 et 12/40, sentence, 6 décembre 2016, $\S \S 488$ et $\mathrm{s}$.

308. CIRDI, World Duty Free Company Limited c. Kenya, n ARB/00/7, sentence, 4 octobre 2006.

309. CIRDI, Getma International, NCT Necotrans, Getma International Investments, NCT Infrastructure \& Logistique c. Guinée, $\mathrm{n}^{\circ} \mathrm{ARB} / 11 / 29$, sentence, 16 août 2016, § 174. Voir aussi supra, I, C (Principes généraux).

310. Ibid., $\S 174-180$.

311. CIRDI, Grupo Francisco Hernando Contreras $S L$ c. Guinée équatoriale, ${ }^{\circ} \mathrm{ARB}(\mathrm{AF}) / 12 / 2$, sentence sur la compétence, 4 décembre 2015 , $\$ \$ 206-210$, examinant si, en droit guinéen, le silence gardé par l'administration peut être assimilé à une acceptation.

312. CIRDI, MNSS BV et Recupero Credito Acciaio NV c. Monténégro, ARB(AF)/12/8, sentence, 4 mai 2016, § 212 ; CPA (CNUDCI), Copper Mesa Mining Corporation c. Équateur, n 201-2, sentence, 15 mars $2016, \S \S 5.63-5.64$. 
ou lors de la réalisation de l'investissement, ils peuvent faire sortir ce dernier du champ matériel des opérations protégées, conduisant le tribunal saisi à se déclarer incompétent pour connaître de la demande de l'investisseur indélicat ${ }^{313}$. Apparus au cours de la gestion d'un investissement légalement constitué, ils ne remettent pas en cause la compétence du tribunal mais peuvent, selon les cas, priver l'investisseur de la protection dont il bénéficie, rendant la requête irrecevable ${ }^{314}$, ou être pris en compte au fond afin d'apprécier la licéité de la réaction de l'État ou la cause du préjudice invoqué ${ }^{315}$, voire être utilisés à l'appui d'une demande reconventionnelle ${ }^{316}$. L'affaire Churchill Mining constitue à cet égard un cas un peu particulier, dans la mesure où ce ne sont pas les conditions de l'investissement lui-même dans une société indonésienne qui étaient contestées mais le point de savoir si ladite société était véritablement titulaire des contrats de concession objets de la demande. Le tribunal ayant établi que ces contrats étaient des faux, il a considéré que cette fraude, et le manque de diligence de l'investisseur à cet égard, entachaient l'ensemble de l'opération et rendaient la demande irrecevable ${ }^{317}$.

\section{b) (Re)structuration de l'investissement}

D'autres motifs peuvent priver le demandeur de la protection dont il se prévaut. Ils tiennent cette fois à la manière dont il a structuré son investissement ou l'a restructuré à l'approche d'un différend.

Une première difficulté peut tenir à la détermination de la nationalité des sociétés demanderesses. Dans ce cas, les tribunaux s'en tiennent classiquement aux critères posés par le traité qui fonde leur compétence, qu'il s'agisse de celui de l'incorporation ${ }^{318}$, éventuellement mâtiné de considérations censées permettre de saisir davantage la réalité économique ${ }^{319}$, ou du siège social ${ }^{320}$. Lorsque c'est ce dernier qui est retenu, il peut être interprété par les arbitres comme visant, en droit international, le siège effectif, mais cette effectivité est appréciée largement, spécialement lorsque le demandeur est une société holding, dont l'objet est de gérer des participations et non de mener des activités opérationnelles régulières ${ }^{321}$. Ainsi, dans le silence des traités, les tribunaux n'offrent guère de résistance aux pratiques

313. CIRDI, Grupo Francisco Hernando Contreras SL c. Guinée équatoriale, $\mathrm{n}^{\circ} \mathrm{ARB}(\mathrm{AF}) / 12 / 2$, sentence sur la compétence, 4 décembre 2015, §§ 258-264.

314. Envisageant cette possibilité, CPA (CNUDCI), Copper Mesa Mining Corporation c. Équateur, $\mathrm{n}^{\circ}$ 201-2, sentence, 15 mars 2016, § 5.62 ; CIRDI, Rusoro Mining Ltd c. Venezuela, $\mathrm{n}^{\circ} \mathrm{ARB}(\mathrm{AF}) / 12 / 15$, sentence, 22 août 2016, $\S 492$, soulignant qu' it is undisputed that claimants with "dirty hands" have no standing in investment arbitration".

315. Trib. Ad hoc (CNUDCI), Oxus c. Ouzbékistan, sentence finale, 17 décembre 2015, § 707 ; CIRDI, MNSS BV et Recupero Credito Acciaio NV c. Monténégro, ARB(AF)/12/8, sentence, 4 mai 2016, § 214 ; CPA (CNUDCI), Copper Mesa Mining Corporation c. Équateur, n 201-2, sentence, 15 mars 2016, §§ 5.54-5.66.

316. Voir infra, C, 1.

317. CIRDI, Churchill Mining PLC et Planet Mining Pty Ltd c. Indonésie, $\mathrm{n}^{\circ} \mathrm{ARB} / 12 / 14$ et 12/40, sentence, 6 décembre 2016, $\S \$ 528-532$.

318. Institut d'arbitrage Ch. comm. Stockholm, Charanne BV et Construction Investment SARL c. Espagne, $\mathrm{n}^{\circ}$ 062/2012, sentence, 21 janvier 2016, $\S \$ 412-418$; CIRDI, MNSS BV et Recupero Credito Acciaio NV c. Monténégro, ARB(AF)/12/8, sentence, 4 mai 2016, $§ 175-179$.

319. CIRDI, Ampal-American Israel Corp. e.a. c. Egypte, $\mathrm{n}^{\circ} \mathrm{ARB} / 12 / 11$, décision sur la compétence, $1^{\text {er }}$ février 2016 , $\S 116$ et s., 196 et s., vérifiant, en application du traité États-Unis/Égypte, que non seulement les sociétés demanderesses ont été incorporées aux États-Unis mais aussi que des ressortissants américains y ont un intérêt substantiel.

320. CIRDI, CEAC Holdings Limited c. Monténégro, $\mathrm{n}^{\circ} \mathrm{ARB} / 14 / 8$, sentence, 26 juillet 2016, $\S 143$ et s., jugeant que les éléments de preuve ne permettent pas d'établir que la société demanderesse avait son siège social à Chypre, quelle que soit la manière dont la notion (comme siège d'immatriculation ou comme siège effectif) est entendue.

321. CIRDI, Tenaris SA and Talta - Trading e Marketing Sociedade Unipessoal Lda. c. Venezuela, $\mathrm{n}^{\circ} \mathrm{ARB} / 12 / 23$, sentence, 29 janvier 2016, $\$$ 197-200 ; CIRDI, Tenaris SA et Talta - Trading e Marketing Sociedade Unipessoal Lda. c. Venezuela, n ARB/12/23, sentence, 12 novembre 2016, §§ 183-190. 
tendant à structurer les investissements de manière à accroître la protection internationale dont ils bénéficient ${ }^{322}$.

Les arbitres se retranchent d'ailleurs derrière la volonté des États, dont ils soulignent qu'ils demeurent libres d'inclure dans leurs traités des clauses de déni des avantages ${ }^{323}$. Celles-ci ont pour objet de permettre aux États d'accueil de refuser le bénéfice de la protection aux sociétés qui n'exercent pas d'activité substantielle sur le territoire de l'État dans lequel elles sont constituées. Mais, même en ce cas, l'appréciation des tribunaux peut demeurer favorable aux demandeurs. Ainsi, la clause de refus des avantages qui figure à l'article 17 du traité sur la charte de l'énergie ${ }^{324}$ n'a pas empêché le tribunal de l'affaire Charanne de se reconnaître compétent pour connaître de la demande formée par des sociétés néerlandaise et luxembourgeoise contre l'Espagne, alors même que cette dernière soutenait que ces sociétés étaient détenues par ses propres ressortissants. De l'avis du tribunal, cette disposition permet seulement aux États de refuser le bénéfice de la protection que le traité octroie aux sociétés contrôlées par les ressortissants d'États tiers, i.e. d'États qui n'y sont pas parties, non à celles qui sont contrôlées par leurs propres nationaux ${ }^{325}$. L'Égypte n'a pas connu davantage de succès lorsqu'elle a prétendu refuser à une société américaine contrôlée par un ressortissant israélien le bénéfice de la protection offerte par un traité États-Unis/Égypte. Ce dernier prévoyait en effet que l'État qui entend user de la clause de déni des avantages doit consulter promptement l'autre État partie, ce que l'Égypte s'était abstenue de faire au cours de la période des six mois de consultations préalable à la saisine du tribunal ${ }^{326}$. Certains demandeurs peuvent certes être pris à leur propre jeu en échouant à prouver l'existence d'un investissement après avoir cherché à le dissimuler aux services fiscaux de leur État de nationalité ${ }^{327}$. Mais il reste que ces solutions ménagent une grande liberté aux investisseurs qui peuvent structurer leurs opérations de manière à bénéficier du régime le plus favorable en cas de différend, même si l'on peut penser que «les efforts de certains négociateurs de TBI pour obtenir que les personnes morales protégées aient un lien réel avec leur pays d'origine seront souvent illusoires tant que les États qu'ils représentent retiendront une conception désincarnée de la personnalité morale dans leur droit interne » ${ }^{28}$.

La liberté des investisseurs n'existe toutefois que pour autant que le différend n'est pas encore né, ou plus précisément que sa survenance n'est pas devenue prévisible. La sentence rendue en l'affaire Philip Morris c. Australie à la fin de l'année 2015 illustre l'application à un cas particulièrement emblématique de

322. Voir aussi CIRDI, RREEF Infrastructure (GP) Limited et RREEF Pan-European Infrastructure Two Lux SARL c. Espagne, n ARB/13/30, décision sur la compétence, 6 juin 2016, $\S 142-147$; CPA (CNUDCI), Flemingo Dutyfree Shop Private Ltd c. Pologne, sentence, 12 août 2016, §§ 312-336, se reconnaissant compétents pour connaître de demandes formulées par des investisseurs indirects membres d'un groupe de sociétés.

323. CIRDI, MNSS BV et Recupero Credito Acciaio NV c. Monténégro, $\mathrm{n}^{\circ} \mathrm{ARB}(\mathrm{AF}) / 12 / 8$, sentence, 4 mai $2016, \S 178$.

324. Aux termes duquel « chaque partie contractante se réserve le droit de refuser le bénéfice de la présente partie [...] à toute entité juridique si les citoyens ou les ressortissants d'un État tiers sont propriétaires ou ont le contrôle de cette entité et si celle-ci n'exerce pas d'activités commerciales substantielles dans la zone de la partie contractante dans laquelle elle est constituée ».

325. Ch. comm. Stockholm, Charanne BV et Construction Investment SARL c. Espagne, ${ }^{\circ}$ 062/2012, sentence, 21 janvier 2016, $\S \S 416-417$, reprenant la solution dégagée dans l'affaire Ioukos (CPA (CNUDCI), Hulley Enterprises Limited (Chypre), Ioukos Universal Limited (Ile de Man), Veteran Petroleum Limited (Chypre) c. Russie, $\mathrm{n}^{\circ}$ AA 226, AA 227 et AA 228, décision sur la compétence et la recevabilité, 30 novembre $2009, \S 415)$.

326. CIRDI, Ampal-American Israel Corp. e.a. c. Égypte, $\mathrm{n}^{\circ} \mathrm{ARB} / 12 / 11$, décision sur la compétence, $1^{\mathrm{er}}$ février 2016, $\$ \$ 124-173$.

327. Ibid., $\S \$ 215-227$.

328. B. REMY, JDI, 2017, n 1, p. 236. 
l'abus consistant à restructurer un investissement dans le seul but de pouvoir soumettre un différend à arbitrage. Pour le tribunal, "the initiation of a treaty-based investor-State arbitration constitutes an abuse of rights (or an abuse of process, the rights abused being procedural in nature) when an investor has changed its corporate structure to gain the protection of an investment treaty at a point in time when a specific dispute was foreseeable " ${ }^{329}$. Tel était le cas en l'espèce : le groupe Philip Morris avait restructuré ses investissements, faisant glisser ses actifs de sa filiale suisse vers sa filiale à Hong-Kong afin de bénéficier du traité en vigueur entre cet État et l'Australie alors qu'il était devenu prévisible que cette dernière adopterait une législation «neutralisant» les emballages de paquets de cigarettes. L' "aléa démocratique " lié aux discussions préalables à l'adoption d'une telle mesure ne saurait remettre en cause sa prévisibilité, et donc celle du différend, d'autant qu'aucune autre raison n'explique de manière convaincante la restructuration opérée ${ }^{330}$. Rendue en 2016 , la sentence de l'affaire Transglobal Green Energy confirme qu'une restructuration opérée alors que le différend porté à la connaissance du tribunal était né ou prévisible doit conduire le tribunal saisi à déclarer la demande irrecevable ${ }^{331}$.

Tous les coups procéduraux ne sont donc pas permis dans l'arbitrage d'investissement. Toutefois, hormis dans ces cas d'abus, la pratique du treaty shopping continue d'avoir de beaux jours devant elle, au moins tant que les États n'auront pu ou voulu y mettre un terme par des clauses conventionnelles habilement rédigées. Tant qu'elle existe, cette pratique peut conduire à des procédures d'arbitrage parallèles. Tel était le cas dans l'affaire Ampal, dans laquelle les investisseurs avaient fait feu de tout bois, initiant plusieurs arbitrages commerciaux sur le fondement des différents contrats qui les liaient à des entreprises égyptiennes ainsi que deux procédures parallèles fondées sur des traités d'investissement, l'une au nom de la société directement en cause, l'autre au nom de son actionnaire principal. Pour le tribunal, un tel activisme ne constitue pas en lui-même un abus. Toutefois, dès lors que, comme c'était le cas en l'espèce, deux tribunaux se sont déclarés compétents pour connaître d'un différend portant sur la même cause, les demandeurs doivent faire un choix sous peine de voir l'abus se cristalliser ${ }^{332}$. Là encore, l'interprétation n'est pas défavorable aux investisseurs, qui se voient offrir la possibilité d'initier plusieurs procédures dans l'espoir qu'un tribunal se déclare compétent, alors que l'article 26 de la convention de Washington prévoit que «le consentement des parties à l'arbitrage dans le cadre de la présente Convention est, sauf stipulation contraire, considéré comme impliquant renonciation à l'exercice de tout autre recours ".

\section{B. Droit applicable}

C'est entendu. Le rôle des tribunaux arbitraux " est de rendre une décision conformément au droit applicable, compte tenu des faits établis. En l'absence

329. CPA (CNUDCI), Philip Morris Asia Ltd. c. Australie, $\mathrm{n}^{\circ}$ 2012-12, sentence sur la compétence et la recevabilité, 17 décembre $2015, \S 554$.

330. Ibid., §§ 555-588.

331. CIRDI, Transglobal Green Energy, LLC et Transglobal Green Panama, SA c. Panama, $\mathrm{n}^{\circ} \mathrm{ARB} / 13 / 28$, sentence, 2 juin 2016, $\S$ 100-119. Voir aussi CIRDI, MNSS BV et Recupero Credito Acciaio NV c. Monténégro, ARB(AF)/12/8, sentence, 4 mai 2016, $\S$ 180-182 et CPA (CNUDCI), Flemingo Dutyfree Shop Private Ltd c. Pologne, sentence, 12 août 2016, $\$$ 337-348, où l'argument est rejeté au motif que les restructurations en cause sont antérieures à la survenance des différends dont les tribunaux sont saisis.

332. CIRDI, Ampal-American Israel Corp. e.a. c. Égypte, $\mathrm{n}^{\circ} \mathrm{ARB} / 12 / 11$, décision sur la compétence,

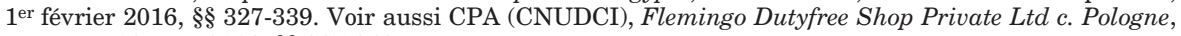
sentence, 12 août 2016 , $\S \S 337-348$. 
d'accord à cet effet entre les Parties, [ils n'ont] pas le pouvoir de statuer ex aequo et bono " 333 . Toutefois, bien que l'erreur sur le droit applicable constitue un excès de pouvoir susceptible de donner lieu à annulation de la sentence rendue, les tribunaux disposent d'une certaine latitude en la matière ${ }^{334}$, qui les conduit à articuler droit interne et droit international suivant des combinaisons variées (1) et à faire une place à diverses sources du droit international (2).

\section{Droit international et droit interne}

Comme chaque année, le millésime 2016 illustre la façon particulière dont s'articulent droit international et droit interne dans le contentieux de l'investissement, laissant apparaître une " construction originale et éphémère, forgée par le tribunal à l'image du différend au règlement duquel elle est dédiée » ${ }^{335}$.

On le sait, le droit international est en principe applicable à la détermination par les tribunaux CIRDI de leur compétence ${ }^{336}$. Celui de l'affaire Pac Rim Cayman en a déduit que l'existence et l'étendue du consentement à l'arbitrage donné par le Salvador dans son code des investissements devaient être appréciées au regard de la convention de Washington, écartant alors les objections tirées de son droit interne par le défendeur ${ }^{337}$. La solution n'était sans doute pas si évidente si l'on veut bien se souvenir des controverses qui entourent l'interprétation des offres d'arbitrage issues des lois nationales, actes formellement internes mais dotés d'effets prévus par le droit international ${ }^{338}$. Mais elle confirme la prégnance du droit international s'agissant de trancher les questions de compétence. Celle-ci n'a toutefois pas empêché les arbitres de l'affaire MNSS BV de donner un certain effet à la renonciation au droit de saisir le mécanisme supplémentaire CIRDI contractuellement consentie par le demandeur. D'un côté, le tribunal a estimé que cette renonciation ne portait que sur les réclamations contractuelles ou plus largement dont l'objet est contractuel, incluant ainsi les réclamations fondées sur la clause de respect des engagements, mais demeurait sans effet sur les réclamations conventionnelles. De l'autre côté, il a estimé qu'un investisseur pouvait fort bien renoncer par contrat aux droits qu'il tire d'un traité d'investissement, dans la mesure où

333. CIRDI, Victor Pey Casado et President Allende Foundation c. Chili, n ARB/98/2, sentence, 13 septembre 2016, § 172. Voir aussi Comité ad hoc CIRDI, Adem Dogan c. Turkménistan, $\mathrm{n}^{\circ}$ ARB/09/9, décision sur l'annulation, 15 janvier 2016, passim (où il est reproché par l'État défendeur au tribunal d'avoir tranché en équité alors qu'il n'en avait pas le pouvoir, ce qui conduit notamment le comité ad hoc à distinguer la mise en œuvre de la clause ex æquo et bono et les considérations d'équité inhérentes à tout système juridique, $\S 99$, ou à noter que l'exercice du pouvoir discrétionnaire d'appréciation des preuves est différent de l'exercice du pouvoir de statuer en équité, § 166). L'équité peut toutefois être occasionnellement invoquée par les tribunaux ou comités ad hoc: voir Comité ad hoc CIRDI, EDF SA et SAUR International $S A$ c. Argentine, $\mathrm{n}^{\circ} \mathrm{ARB} / 03 / 23$, décision sur l'annulation, 5 février 2016, $\$ 73$ (diverses considérations, dont certaines relatives à l'équité, peuvent conduire un comité $a d$ hoc à ne pas annuler une sentence pourtant viciée) ; CPA (CNUDCI), Copper Mesa Mining Corporation c. Équateur, n 201-2, sentence, 15 mars 2016 , $\S 9.10$ (où le tribunal retient la "fairest decision " en matière de répartition des coûts).

334. Comité ad hoc CIRDI, Total SA c. Argentine, $\mathrm{n}^{\circ} \mathrm{ARB} / 04 / 01$, décision sur l'annulation, $1^{\text {er }}$ février 2016, §§ 186-222, spéc. §§ 197 et 217.

335. Raphaële RIVIER, «L'articulation entre droit national et droit international devant les tribunaux arbitraux internationaux d'investissement ", in Sabrina ROBERT-CUENDET (dir.), Droit des investissements internationaux. Perspectives croisées, Bruxelles, Bruylant, 2017, p. 414.

336. CIRDI, Vestey Group Limited c. Venezuela, $\mathrm{n}^{\circ} \mathrm{ARB} / 06 / 4$, sentence, 15 avril 2016, $\S 115$; CIRDI, Corona Materials, LLC c. République dominicaine, ARB(AF)/14/3, sentence sur l'exception préliminaire, 31 mai 2016, §§ 185-187.

337. CIRDI, Pac Rim Cayman LLC c. République du Salvador, $\mathrm{n}^{\circ}$ ARB/09/12, sentence, 14 octobre 2016 , $\S \S 5.68-5.73$, le Salvador soutenant alternativement que son code minier donne compétence exclusive aux juridictions internes ou que son code civil rend forcloses les demandes formulées.

338. Voir cette chronique, cet Annuaire, 2010, p. 626 ; 2011, p. $433 ; 2012$, p. 618 ; 2013, p. 439 ; 2014, p. $562 ; 2015$, pp. $861-862$. 
cette renonciation ne met pas en cause l'intérêt public poursuivi par les États qui lui ont reconnu par traité lesdits droits ${ }^{339}$. C'est là une autre manifestation du fait que les traités d'investissement confèrent bien des droits, au moins procéduraux, aux investisseurs, dont ils peuvent disposer dans la mesure où cela ne remet pas en cause l'intérêt public ${ }^{340}$.

Affirmer que le droit international est applicable à la détermination par les tribunaux de leur compétence ne les empêche toutefois pas de prendre en compte le droit interne, comme " donnée à établir ", notamment "à enregistrer » ${ }^{341}$, par exemple lorsque la propriété ou l'existence de l'investissement est contestée ${ }^{342}$, ou comme "état à considérer ", notamment lorsque la compétence du tribunal dépend de la conformité de l'investissement au droit interne ${ }^{343}$. Mais, en droit des investissements peut-être plus qu'ailleurs, les choses sont rarement simples. Ainsi, la nationalité du demandeur est généralement considérée comme l'une des questions qui doit être tranchée sur la base du droit interne, en vertu d'un renvoi du droit international. Or, en l'affaire Tenaris, dans laquelle les traités pertinents retenaient les critères de l'incorporation et du siège social pour identifier les personnes morales protégées, le tribunal a remarqué que tandis que l'appréciation du premier mobilisait nécessairement le droit interne, celle du second n'impliquait pas pareil renvoi. Dès lors, "the interpretation of the terms "siège social" or "sede" is a matter of international, not domestic, law »... ce qui n'a pas empêché les arbitres de tenir compte des droits internes concernés en tant que moyen complémentaire d'interprétation ! 344

$\mathrm{Au}$ fond, la question paraît plus délicate encore. Bien que les parties, et subsidiairement les tribunaux, disposent alors en principe d'une liberté dans la détermination du droit applicable, la pratique tend à indiquer que « le droit applicable suit la cause juridique de la réclamation ${ }^{345}$. Ainsi, lorsque la compétence du tribunal est fondée sur une loi interne dont le demandeur met en cause la violation par l'État, le droit interne est applicable et le droit international ne joue qu'un rôle complémentaire. Tel était le cas dans l'affaire Pac Rim Cayman, dans laquelle les arbitres ont estimé que le refus par le Salvador de délivrer le permis minier sollicité par l'investisseur était conforme au droit minier salvadorien. Pour le déterminer, ils durent interpréter le droit interne en accordant une place particulière aux positions adoptées par les autorités salvadoriennes avant de souligner tout de même que

339. CIRDI, MNSS BV et Recupero Credito Acciaio NV c. Monténégro, $\mathrm{n}^{\circ} \mathrm{ARB}(\mathrm{AF}) / 12 / 8$, sentence, 4 mai $2016, \S \S 148-165$, dans la mesure où la renonciation prive l'investisseur de la possibilité de se prévaloir de la clause de respect des engagements figurant dans le traité.

340. Pour une analyse de la question dans un autre contexte, voir Evelyne LAGRANGE, « L'application des accords relatifs à l'investissement dans les ordres juridiques internes ", in Sabrina ROBERT-CUENDET (dir.), Droit des investissements internationaux. Perspectives croisées, op. cit., pp. 494 et s.

341. Ces classifications sont empruntées à Raphaële RIVIER, op. cit.

342. CIRDI, Vestey Group Limited c. Venezuela, ${ }^{\circ}$ ARB/06/4, sentence, 15 avril 2016, §§ 193-201; CIRDI, Churchill Mining PLC et Planet Mining Pty Ltd c. Indonésie, ${ }^{\circ}$ ARB/12/14 et 12/40, sentence, 6 décembre 2016, § 235. Voir également CIRDI, Joseph Houben c. Burundi, $\mathrm{n}^{\circ} \mathrm{ARB} / 13 / 7$, sentence, 12 janvier 2016, $\S 99$, soulignant la difficulté que le tribunal a eu à déterminer la situation juridique du terrain en cause en droit burundais.

343. S'agissant de la conformité au droit local, voir les exemples mentionnés supra B, 1 et Comité ad hoc CIRDI, Adem Dogan c. Turkménistan, n ARB/09/9, décision sur l'annulation, 15 janvier 2016, $\S \S 125-136$, soulignant que le tribunal a fait, comme il le devait, application du droit interne pour trancher la question.

344. CIRDI, Tenaris SA and Talta - Trading e Marketing Sociedade Unipessoal Lda. c. Venezuela, $\mathrm{n}^{\circ} \mathrm{ARB} / 12 / 23$, sentence, 29 janvier 2016, §§ 163-195, spé. $\$ 165$.

345. Raphaële RIVIER, op. cit., p. 482. En ce sens, Comité ad hoc CIRDI, EDF SA et SAUR International SA c. Argentine, $\mathrm{n}^{\circ} \mathrm{ARB} / 03 / 23$, décision sur l'annulation, 5 février 2016, $§ 219$. 
l'interprétation retenue n'écartait pas le droit salvadorien des solutions privilégiées au sein de nombreux États ${ }^{346}$.

À l'inverse, lorsque le demandeur se plaint de la violation par l'État d'accueil de ses obligations au titre d'un traité d'investissement, le droit international devient applicable, et le droit interne est seulement pris en considération à la manière d'un fait. Dès lors, toute violation du droit interne n'emporte pas nécessairement violation du droit international, spécialement du traité pertinent ${ }^{347}$ et, réciproquement, le droit interne ne saurait justifier le non-respect par l'État de ses obligations au titre du droit international ${ }^{348}$. Cela étant, la prise en considération du droit interne peut être décisive. Tel peut être le cas lorsque le demandeur se plaint d'une expropriation internationalement illicite, dont l'identification peut impliquer de déterminer s'il disposait bien de droits en tant que titulaire d'une marque protégée en droit interne ${ }^{349}$ ou d'apprécier si la procédure interne de nationalisation a été respectée ${ }^{350}$. L'intrication des droits international et interne est plus forte encore en présence d'une clause de respect des engagements. Sur ce point, le tribunal de l'affaire Garanti Koza a soutenu que l'existence desdits engagements devait s'apprécier en application du droit interne mais que leur violation devait s'analyser sur la base du droit international. Dès lors, l'Etat défendeur ne saurait invoquer son droit interne pour justifier la violation par diverses entités dont le comportement lui est imputable en vertu du droit international de l'engagement contractuellement pris en son nom de régler les travaux réalisés de manière forfaitaire et non en fonction de leur avancement ${ }^{351}$. Cette insistance sur le droit international rend d'autant plus surprenante la référence au contrat opérée pour refuser l'application d'intérêts composés à la somme due au titre de la violation de la clause de respect des engagements ${ }^{352}$.

\section{Traités d'investissements et autres instruments internationaux}

Les accords de promotion et de protection des investissements ne constituent pas la seule source du droit international à laquelle puisent les tribunaux afin de trancher les différends qui leur sont soumis. Comme c'est désormais régulièrement

346. CIRDI, Pac Rim Cayman LLC c. République du Salvador, $\mathrm{n}^{\circ}$ ARB/09/12, sentence, 14 octobre 2016, $\S \S 5.60-5.65$ sur le droit applicable, $\$ \$ 8.2-8.69$ pour l'application de la loi salvadorienne.

347. CIRDI, Urbaser SA et Consorcio de Aguas Bilbao Bizkaia, Bilbao Biskaia Ur Partzuergoa c. Argentine, $\mathrm{n}^{\circ} \mathrm{ARB} / 07 / 26$, sentence, 8 décembre 2016, par ex. § 146. Voir également CIRDI, MNSS BV et Recupero Credito Acciaio NV c. Monténégro, ARB(AF)/12/8, sentence, 4 mai 2016, $\S 308-310$.

348. Comité ad hoc CIRDI, Total SA c. Argentine, $\mathrm{n}^{\circ} \mathrm{ARB} / 04 / 01$, décision sur l'annulation, $1^{\mathrm{er}}$ février 2016, §§ 212-222 ; Comité ad hoc CIRDI, EDF SA et SAUR International SA c. Argentine, ${ }^{\circ}$ ARB/03/23, décision sur l'annulation, 5 février 2016, $\$$ 218-222, confirmant que l'état d'urgence décrété en Argentine ne peut, en tant que tel, justifier la violation des obligations résultant des engagements internationaux de l'État.

349. CIRDI, Philip Morris Brands SARL, Philip Morris Products SA c. Uruguay, n ARB/10/7, sentence, 8 juillet $2016, \S \S 236-271$.

350. CIRDI, Tenaris SA and Talta - Trading e Marketing Sociedade Unipessoal Lda. c. Venezuela, $\mathrm{n}^{\circ} \mathrm{ARB} / 12 / 23$, sentence, 29 janvier 2016, $\S \S 481-496$. Voir aussi Comité ad hoc CIRDI, TECO Guatemala Holdings LLC c. Guatemala, n ${ }^{\circ} \mathrm{ARB} / 10 / 23$, décision sur l'annulation, 5 avril 2016, $\S \S 308-323$, s'agissant d'un cas dans lequel la violation du droit interne est prise en considération pour identifier une violation du standard de traitement juste et équitable.

351. CIRDI, Garanti Koza LLP c. Turkménistan, n ARB/11/20, sentence, 19 décembre 2016, $\S \S 328-354$, spé. $\S \S 331-332$ pour le droit applicable et $\$ \S 351-353$ pour le constat de la violation des engagements internes en application du droit international. Voir aussi, sur la portée de la clause de respect des engagements, Trib. Ad hoc (CNUDCI), Oxus c. Ouzbékistan, sentence finale, 17 décembre 2015, \$§ 364 et s.

352. Ibid., § 436. Voir CIRDI, Saint-Gobain Performance Plastics Europe c. Venezuela, n ARB/12/13, décision sur la responsabilité et les principes de la réparation, 30 décembre $2016, \S 890$, écartant l'application du droit vénézuélien au profit du droit international s'agissant d'apprécier les intérêts dans la mesure où c'est la responsabilité internationale de l'État qui est en cause. 
le cas, les arbitres ont cherché en 2016 à articuler les droits et obligations résultant de ces accords avec d'autres traités internationaux ainsi qu'avec le droit de l'Union européenne.

La réserve dont font généralement preuve les arbitres à l'heure de mobiliser des sources internationales autres que les traités qui fondent leur compétence a peut-être fait long feu. Certes, la façon dont les tribunaux configurent les faits peut suffire à écarter les arguments qui font appel à des sources externes pour justifier certains comportements. Ainsi, le Burundi ne saurait soutenir que le droit international des droits de l'homme l'empêche d'expulser ceux qui ont établi leur domicile sur le terrain d'un investisseur alors que ce qui lui est reproché est d'avoir laissé initialement ces personnes pénétrer sur ces terres ${ }^{353}$. Toutefois, l'issue de deux affaires tranchées dans l'année tient pour une bonne part à la prise en compte de sources externes.

Le tribunal de l'affaire Urbaser a ainsi pris le contre-pied de plusieurs sentences rendues contre l'Argentine en insistant sur le fait que le droit d'accès à l'eau, garanti aux niveaux constitutionnel et international, constituait l'un des éléments clefs du cadre juridique applicable à une opération d'investissement dans le secteur de l'assainissement et de la distribution d'eau potable. Dès lors, un investisseur ne saurait attendre légitimement d'un État qu'il s'abstienne de prendre les mesures exigées afin de mettre en œuvre ce droit, mesures qui, en tout état de cause, seraient justifiées par un état de nécessité ${ }^{354}$. L’approche générale du tribunal sur la question apparaît plus nettement encore lorsqu'il se prononce sur la demande reconventionnelle formulée par l'Argentine contre l'investisseur : de l'avis des arbitres, le traité pertinent «is not framed in isolation, but placed in the overall system of international law» et l'article $31, \S 3, \mathrm{c}$ ) de la convention de Vienne sur le droit des traités implique quoi qu'il en soit qu'il soit "construed in harmony with other rules of international law of which it forms part, including those relating to human rights $» 355$.

L'affaire Philip Morris c. Uruguay manifeste également cette perméabilité du contentieux transnational à la diversité des sources du droit international. Le tribunal a considéré, d'abord, que les dispositions du traité pertinent devaient être interprétées à la lumière du droit coutumier, établi en partie en se référant à des sources externes, dont l'Accord économique et commercial global conclu entre l'Union européenne et le Canada mais pas encore en vigueur ${ }^{356}$; ensuite, que la doctrine de la marge d'appréciation dégagée par la Cour européenne des droits de l'homme pouvait être également appliquée dans le domaine des investissements ${ }^{357}$; enfin, que la convention-cadre de l'OMS sur la lutte contre le tabac constituait un "point of reference " pour apprécier le caractère raisonnable des mesures prises

353. CIRDI, Joseph Houben c. Burundi, n ARB/13/7, sentence, 12 janvier 2016, § 177 ; voir aussi § 235 où le tribunal indique toutefois que la restitution, qui impliquerait l'expulsion, n'est plus envisageable. Voir aussi CIRDI, Vestey Group Limited c. Venezuela, n ARB/06/4, sentence, 15 avril 2016, $\S 297$ et s., estimant que les mesures prises par le défendeur n'ont pas contribué à la réalisation du droit à l'alimentation. 354. CIRDI, Urbaser SA et Consorcio de Aguas Bilbao Bizkaia, Bilbao Biskaia Ur Partzuergoa c. Argentine, $\mathrm{n}^{\circ} \mathrm{ARB} / 07 / 26$, sentence, 8 décembre 2016, $\$ \$ 622$ et s., 720 et s., reprenant le raisonnement sous l'angle de l'état de nécessité.

355. Ibid., $\S \S 1200-1201$, reprenant la position du comité ad hoc de l'affaire Tulip Real Estate c. Turquie ( $\mathrm{n}^{\circ} \mathrm{ARB} / 11 / 28$, décision sur la demande d'annulation, 30 décembre 2015, $\S \S 86-92$ ). Voir aussi supra, I, A, 1 (interprétation des traités) et infra, C.

356. CIRDI, Philip Morris Brands SARL, Philip Morris Products SA c. Uruguay, n ARB/10/7, sentence, 8 juillet 2016 , $\$ \$ 287-301$ s'agissant d'interpréter la notion d'expropriation en y incluant la doctrine des police powers ; $\S 316-324$, s'agissant d’interpréter la notion de traitement juste et équitable. 357. Ibid., § 399 . 
par l'État défendeur, alors même que l'État de nationalité du demandeur n'y était pas partie ${ }^{358}$.

Ces deux décisions indiquent tout à la fois que des sources internationales autres que les traités d'investissement peuvent jouer un rôle majeur dans le contentieux arbitral transnational et qu'elles peuvent le faire de plusieurs manières ${ }^{359}$. Dans ces deux cas, il ne s'agit pas d'appliquer des traités distincts mais d'en tenir compte, tantôt afin d'interpréter les dispositions du traité appliqué, tantôt comme élément de contexte permettant d'apprécier la conformité à ce traité des comportements étatiques.

Plusieurs décisions rendues en 2016 sont également revenues sur l'articulation des rapports entre les traités d'investissement et le droit de l'Union européenne. Sur ce point, le comité ad hoc saisi en l'affaire Micula n'a rien trouvé à redire sur la place que le tribunal qui avait statué dans cette affaire avait réservé au droit européen dans son raisonnement. Pour mémoire, ce dernier avait jugé l'accord d'association entre la Roumanie et la Communauté européenne conclu en 1995 applicable mais avait refusé d'en déduire que l'ensemble du droit de l'Union européenne devait être appliqué, n'en tenant compte qu'au titre de la "factual matrix » du différend. Il avait alors jugé que la remise en cause par la Roumanie de l'aide fiscale dont bénéficiait le demandeur n'était pas déraisonnable en ce qu'elle se justifiait par l'objectif d'accession à l'Union européenne mais qu'elle violait le standard de traitement juste et équitable à trois égards : elle remettait en cause les attentes légitimes de l'investisseur, nées des engagements pris par la Roumanie alors que la perspective de son adhésion à l'Union était déjà proche ; elle manquait de transparence, l'État défendeur ayant tardé à informer l'investisseur après s'être aperçu de l'incompatibilité de ce régime avec le droit de l'UE ; elle s'accompagnait d'un maintien des obligations pesant sur l'investisseur en contrepartie de l'aide dont il bénéficiait. Selon le comité, le tribunal ne s'est rendu coupable ni d'un excès de pouvoir ni d'un défaut de motifs en jugeant que, dans ces conditions, le retrait des aides violait le standard de traitement juste et équitable puis en refusant de se prononcer sur les conséquences de ce constat en termes d'exécution ${ }^{360}$. Le comité insiste ainsi sur le fait que le tribunal ne s'est pas prononcé sur la conformité de la décision roumaine au droit de l'UE, pas plus que sur la compatibilité de ce dernier avec le TBI pertinent. Il s'est uniquement prononcé sur le comportement de l'État défendeur, qui ne se trouvait donc pas pris dans un conflit d'obligations. Reste que la Roumanie se trouve désormais face à un tel conflit puisqu'elle est tenue d'exécuter la sentence en application de la convention de Washington et ne saurait le faire sans violer le droit de l'UE. La solution de ce conflit ne viendra donc pas de la procédure d'annulation. Si la Cour de Justice de l'Union européenne, saisie de la décision de la Commission européenne selon laquelle l'exécution de la sentence constituerait une aide d'État illégale ${ }^{361}$, confirme cette position, le conflit ne pourra se régler que sur le terrain politique.

358. Ibid., § 401. Voir supra, I, B (Coutume) et D (Sources informelles).

359. Pour une recension des fondements de la prise en considération des sources internationales externes, voir Patrick JACOB, «La place des normes externes dans le contentieux de l'investissement ", in Sabrina RoBerT-CuEndet (dir.), Droit des investissements internationaux. Perspectives croisées, op. cit., pp. 607-640 et, relativisant l'importance de ce fondement, Franck LATTY, "Le point de vue du droit international des investissements ", in SFDI, La mise en œuvre de la lex specialis dans le droit international contemporain, Paris, Pedone, 2017, pp. 117-131.

360. Comité ad hoc CIRDI, Ion Micula et al. c. Roumanie, $\mathrm{n}^{\circ} \mathrm{ARB} / 05 / 20$, décision sur l'annulation, 26 février 2016, §§ 178-203 et 225-235.

361. CJUE, Micula et autres c. Commission, aff. T-104/15, recours en annulation introduit le 28 novembre 2015. 
Les décisions rendues par deux nouveaux tribunaux confrontés à des différends intra-européens dans des conditions similaires confirment l'indifférence du contentieux arbitral transnational aux problématiques spécifiques du droit de l'Union. Ces tribunaux étaient tous deux saisis de demandes émanant d'investisseurs néerlandais et luxembourgeois mettant en cause la conformité avec le traité sur la charte de l'énergie des modifications apportées à partir de 2011 par l'Espagne à son cadre légal et réglementaire de soutien à l'industrie photovoltaïque. Pour l'État défendeur, soutenu par la Commission européenne ${ }^{362}$, ces demandes devaient être déclarées irrecevables pour trois raisons : elles émaneraient d'investisseurs européens, et donc pas d'investisseurs d'une autre partie contractante au sens du traité sur la charte de l'énergie ; ce dernier contiendrait une «clause de déconnexion » implicite, selon laquelle il ne s'appliquerait pas dans les relations entre États membres de l'Union; le monopole reconnu à la Cour de justice de l'Union par l'article 344 TFUE s'opposerait à l'exercice par les tribunaux de leur compétence. De manière désormais classique, les deux tribunaux ont écarté l'ensemble de ces arguments. Celui de l'affaire Charanne a insisté sur le fait que, si un investisseur européen ne pouvait agir contre l'Union elle-même, les investisseurs d'États membres pouvaient fort bien mettre en cause les décisions prises par d'autres États membres, faisant ainsi apparaître le dédoublement des espaces en territoires des États membres et de l'Union ${ }^{363}$. Les deux ont souligné que le TCE ne contenait aucune clause de déconnexion, même implicite ${ }^{364}$; que l'article 344 TFUE ne réservait pas à la Cour de justice l'ensemble des différends opposant les ressortissants d'un État membre à un autre État membre ${ }^{365}$; et qu'ils n'étaient donc pas confrontés à un véritable conflit d'obligations ${ }^{366}$. Mais le tribunal de l'affaire RREEF Infrastructure a en outre tenu à souligner que, quand bien même un tel conflit apparaîtrait et ne pourrait être résolu par une interprétation harmonieuse, du point de vue du droit international, "EU law does not and cannot "trump" public international $l a w » 367$. Autrement dit, le principe de primauté reconnu au droit de l'Union ne joue que dans l'ordre européen, tandis que, du point de vue du droit international, le droit de l'Union apparaît comme une source ordinaire que le principe de spécialité commande au tribunal constitué sur le fondement d'un traité spécifique auquel sont parties des États membres de l'UE comme des États non membres tel que le traité sur la charte de l'énergie d'écarter au profit de ce dernier ${ }^{368}$. Bien que, ce disant, le tribunal souligne son accord avec le tribunal de l'affaire Electrabel, on peut douter que cet accord soit parfait. En effet, après avoir, comme en l'espèce, ravalé le droit de l'UE au rang de droit international « ordinaire » et jugé que l'articulation des différentes sources du droit international devait se faire en application des règles

362. La Commission européenne a pu présenter un mémoire d'amicus curiae dans l'affaire Charanne (Institut d'arbitrage Ch. comm. Stockholm, Charanne BV et Construction Investment SARL c. Espagne, $\mathrm{n}^{\circ} 062 / 2012$, sentence, 21 janvier 2016 , $\$ 56$-60 et $\left.\S 425\right)$ mais sa demande d'intervention a été rejetée dans l'affaire RREEF (CIRDI, RREEF Infrastructure (GP) Limited et RREEF Pan-European Infrastructure Two Lux SARL c. Espagne, $\mathrm{n}^{\circ} \mathrm{ARB} / 13 / 30$, décision sur la compétence, 6 juin 2016 , $\$ 20$ ). Pour d'autres interventions, voir CPA (CNUDCI), Mesa Power Group, LLC c. Canada, $\mathrm{n}^{\circ} 2012-17$, sentence, 24 mars 2016 et CPA, Windstream Energy LLC c. Canada, $\mathrm{n}^{\circ}$ 2013-22, sentence, 27 septembre 2016, mentionnant les interventions des États parties à l'ALENA non parties au différend; CIRDI, Pac Rim Cayman LLC c. République du Salvador, $\mathrm{n}^{\circ} \mathrm{ARB} / 09 / 12$, sentence, 14 octobre 2016, §§ 3.28-3.30, mentionnant un amicus curiae du Center for International Environnemental Law sans en tenir compte.

363. Institut d'arbitrage Ch. comm. Stockholm, Charanne BV et Construction Investment SARL c.

Espagne, $\mathrm{n}^{\circ}$ 062/2012, sentence, 21 janvier 2016, $\S$ 427-432.

364. Charanne BV, $\S \S 433-438$; RREEF Infrastructure, $\S \S 81-86$.

365. Charanne $B V, \S \S 440-450$; RREEF Infrastructure, $\$ \S 78-80$.

366. Charanne BV, $\S 438 ;$ RREEF Instrastructure, $\S 87$.

367. CIRDI, RREEF Infrastructure (GP) Limited et RREEF Pan-European Infrastructure Two Lux

SARL c. Espagne, $\mathrm{n}^{\circ} \mathrm{ARB} / 13 / 30$, décision sur la compétence, 6 juin 2016, $\$ 87$.

368. Ibid., $\$ 75$. 
de ce dernier, les arbitres de l'affaire Electrabel avaient tout de même considéré que, dans l'hypothèse où une contradiction apparaîtrait entre le traité sur la charte de l'énergie et le droit de l'UE, ce dernier devait prévaloir dans les rapports entre États membres en application de la règle internationale de conflit applicable, que le tribunal avait tiré de l'article 351 TFUE ${ }^{369}$. Celui de l'affaire RREEF n'en tient pas compte, considérant qu'il doit prioritairement appliquer le traité sur la base duquel il est constitué et qui forme donc sa « constitution » 370 .

\section{Incidents de procédures et procédures incidentes}

\section{Demandes reconventionnelles}

Le déséquilibre du contentieux arbitral transnational relatif à l'investissement, dans lequel les tribunaux sont systématiquement saisis par des personnes privées cherchant à engager la responsabilité des États, est régulièrement dénoncé. La technique des demandes reconventionnelles pourrait alors conduire à rééquilibrer le rapport de force, en permettant aux États défendeurs de mettre à leur tour en cause les investisseurs. Les limites mises à la compétence des tribunaux font toutefois douter de cette possibilité, malgré l'audace de certains arbitres, illustrée cette année par la sentence rendue en l'affaire Urbaser.

Bien souvent, les tribunaux arbitraux se déclarent incompétents pour connaître de la demande reconventionnelle formée par l'État défendeur. Certes, la possibilité d'introduire de telles demandes est envisagée dans le contentieux arbitral, ainsi que l'indique l'article 46 de la convention de Washington, aux termes duquel « le Tribunal doit [...] statuer sur toutes demandes incidentes, additionnelles ou reconventionnelles se rapportant directement à l'objet du différend ». Mais encore faut-il, poursuit cette disposition, "que ces demandes soient couvertes par le consentement des parties et qu'elles relèvent par ailleurs de la compétence du Centre ». Or, lorsque la compétence d'un tribunal est fondée sur un traité, elle est parfois restreinte aux différends relatifs à l'application dudit traité, qui ne fait peser d'obligations que sur les États parties. C'est ce qui explique le rejet par les tribunaux des affaires Vestey ${ }^{371}$ et Rusoro ${ }^{372}$ des demandes reconventionnelles formulées par le Venezuela. Le raisonnement retenu par le tribunal de l'affaire Oxus est à peine moins tranché. Ce dernier s'est également déclaré incompétent pour connaître de la demande reconventionnelle formée par l'Ouzbékistan en soulignant qu'il tirait sa compétence d'un traité prévoyant que les différends relatifs au respect par les États parties de leurs obligations en vertu de ce traité pouvaient être soumis à arbitrage ${ }^{373}$. Mais il a également envisagé timidement l'idée selon laquelle "as soon as [a tribunal] has jurisdiction over a claim, it has jurisdiction over a counter-claim closely linked to that claim ${ }^{374}$. Cette concession était cependant sans conséquence dès lors que le tribunal a estimé tantôt que la demande reconventionnelle n'était pas étroitement liée à la demande principale tantôt qu'elle était

369. CIRDI, Elecrabel SA c. Hongrie, n ARB/07/19, décision sur la compétence, le droit applicable et la responsabilité, 30 novembre 2012, § 4.189, cette chronique, cet Annuaire, 2012, p. 649. Voir aussi Franck LATTY, «Le point de vue du droit international des investissements », op. cit., p. 131, jugeant "plus convaincante " l'approche du tribunal de l'affaire RREEF.

370. Décision RREEF, §75.

371. CIRDI, Vestey Group Limited c. Venezuela, n ARB/06/4, sentence, 15 avril 2016, § 333, même si le cas était particulier car la demande tendant à obtenir le titre sur la propriété de l'investisseur n'était pas explicitement présentée comme reconventionnelle.

372. CIRDI, Rusoro Mining Ltd c. Venezuela, $\mathrm{n}^{\circ} \mathrm{ARB}(\mathrm{AF}) / 12 / 15$, sentence, 22 août 2016, §§ 618-633.

373. Trib. ad hoc (CNUDCI), Oxus c. Ouzbékistan, sentence finale, 17 décembre 2015, §§ 937-951.

374. Ibid., § 952. 
de nature contractuelle et devait dès lors être soumise au juge du contrat. Difficile dès lors de considérer cette incise comme un pas dans la direction d'une admission plus large des demandes reconventionnelles sur la seule base de l'étroite connexité qu'elles entretiendraient avec la demande principale.

En revanche, le raisonnement du tribunal de l'affaire Urbaser doit être souligné et intéressera largement les spécialistes de droit international public. Ce tribunal pouvait s'appuyer sur une clause d'arbitrage largement rédigée puisqu'elle lui donnait compétence pour connaître de tout différend relatif à un investissement. Il en déduisit qu'il pouvait connaître aussi bien des violations du traité que d'autres demandes et considéra que l'investisseur ne pouvait restreindre la portée de la clause d'arbitrage sur laquelle il entendait se fonder sans remettre en cause la rencontre des consentements. La demande reconventionnelle formée par l'Argentine entrait donc dans le champ matériel de la compétence du tribunal dès lors qu'elle était bien relative à un investissement ${ }^{375}$. Restait alors à déterminer si les autres conditions de recevabilité d'une demande reconventionnelle étaient réunies. Alors que d'autres demandes avaient pu se heurter à l'obstacle de la connexité, spécialement juridique, le tribunal jugea ici que celle-ci était établie dans la mesure où la demande argentine s'appuyait non sur le droit interne mais, comme la demande principale, sur le droit international. L'Argentine mettait en effet en cause l'investisseur pour violation de ses obligations internationales en matière d'accès à l'eau 376 !

Ainsi motivée par des contraintes procédurales, cette configuration de la demande reconventionnelle allait mener les arbitres fort loin au fil d'un raisonnement tortueux qui peut être résumé en quatre temps. 1) Des sources variées indiquent que les personnes privées, dont les investisseurs, sont des sujets du droit international. Elles bénéficient de droits mais peuvent également être soumises à des obligations en vertu du droit international. Ainsi, les personnes privées sontelles internationalement tenues de ne pas porter atteinte à la dignité de la personne humaine non plus qu'au droit à des conditions de vie et de logement décentes ${ }^{377}$. 2) Les droits de l'homme internationalement reconnus font partie du cadre juridique applicable à l'opération d'investissement en vertu du traité bilatéral d'investissement et peuvent donc être invoqués à l'appui d'une demande reconventionnelle ${ }^{378}$. 3) Toutefois, ni le traité ni le droit international général ne font peser sur l'investisseur l'obligation positive d'assurer l'accès des populations à l'eau potable. Cette obligation pèse sur l'État, qui lui donne effet en imposant certaines obligations à son concessionnaire ${ }^{379}$. 4) Alors même que ce dernier n'aurait pas respecté l'ensemble de ses obligations contractuelles, il n'en a pas pour autant violé une prétendue obligation internationale de favoriser l'accès des populations à l'eau potable, de sorte que la demande reconventionnelle doit être rejetée ${ }^{380}$.

375. CIRDI, Urbaser SA et Consorcio de Aguas Bilbao Bizkaia, Bilbao Biskaia Ur Partzuergoa c. Argentine, $\mathrm{n}^{\circ} \mathrm{ARB} / 07 / 26$, sentence, 8 décembre 2016, $\S \S 1143-1148$, s'agissant d'apprécier la compétence du tribunal pour connaître de la demande reconventionnelle, $\$ \S 1182-1192$, s'agissant de déterminer le droit applicable à cette demande, qui inclut non seulement le traité mais aussi les autres sources du droit international.

376. Ibid., § 1151.

377. Ibid., §§ 1193-1199, le tribunal s'appuyant sur le pacte international sur les droits économiques, sociaux et culturels, mais aussi la déclaration universelle des droits de l'homme, la déclaration tripartite de l'OIT sur les entreprises multinationales et la politique sociale ou encore les principes directeurs relatifs aux entreprises et aux droits de l'homme du Conseil des droits de l'homme. Sur l'usage de la soft law, voir supra, I, D (Sources informelles).

378. Ibid., §§ 1200-1203 et 1207.

379. Ibid., $\S 1204-1210$.

380. Ibid., §§ 1211-1221. 
Malgré cette conclusion, le raisonnement ici tenu est de nature à encourager les demandes reconventionnelles et s'inscrit dans une perspective de rééquilibrage du contentieux de l'investissement en soulignant que les investisseurs peuvent, eux aussi, être soumis à des obligations internationales dont le respect peut être discuté à l'occasion d'un arbitrage ${ }^{381}$. Pour y parvenir, les arbitres doivent toutefois se livrer à d'étonnantes contorsions. À les en croire, lorsqu'un tribunal est saisi à titre principal de la violation d'un traité sur la base d'une clause lui donnant compétence pour connaître de tout différend relatif à un investissement - et dans ce cas seulement, il peut connaître des demandes reconventionnelles mettant en cause la violation par le demandeur de ses propres obligations internationales. D'un côté, ce raisonnement pourrait conduire à un élargissement peu contrôlable de la compétence matérielle des tribunaux arbitraux, dès lors susceptibles d'être saisis de toute demande en relation avec un investissement, quelle qu'en soit la cause juridique. De l'autre, il participe à l'affirmation de la personnalité internationale des personnes privées et en particulier à la soumission des entreprises à des obligations internationales, sorte de contrepartie aux droits qui leur sont reconnus en vertu des traités d'investissement ${ }^{382}$. Sur ce dernier point, on remarquera que les Principes directeurs relatifs aux entreprises et aux droits de l'homme du Conseil des droits de l'homme (également appelés Principes Ruggie, du nom du rapporteur) sont mobilisés, non seulement pour trouver la trace de la soumission des entreprises à des obligations internationales en la matière mais également, même si cela n'est pas dit, pour distinguer l'obligation, positive, de l'État de protéger ces droits de celle, négative, de l'investisseur de les respecter ${ }^{383}$.

\section{Demandes de récusation}

Comme chaque année, plusieurs demandes de récusation ont été formulées, essentiellement cette année par les États défendeurs, spécialement par le Venezuela. Elles ont toutes été rejetées.

Sur le plan factuel, deux points peuvent être soulignés. On remarquera d'abord que la procédure de récusation peut être utilisée à des fins détournées, comme lorsque les demandeurs de l'affaire $B S G$ Resources ont cherché à récuser, sans succès, l'ensemble des membres du tribunal après que, et parce que, celui-ci avait refusé d'ordonner pour la deuxième fois à la Guinée la production de certains documents ${ }^{384}$. On observera ensuite que pas moins de quatre décisions portent sur des demandes formulées par le Venezuela et sont dirigées contre un même arbitre dont

381. On remarquera que moins de deux mois après cette décision, une demande reconventionnelle a abouti pour la première fois à la condamnation d'un investisseur (CIRDI, Burlington Resources c. Équateur, $\mathrm{n}^{\circ} \mathrm{ARB} / 08 / 5$, décision sur les demandes reconventionnelles, 7 février 2017). Cette décision sera commentée dans la prochaine livraison de cette chronique (voir déjà CIRDI, Perenco Ecuador Limited c. Équateur, $\mathrm{n}^{\circ} \mathrm{ARB} / 08 / 6$, décision intermédiaire sur la demande reconventionnelle relative à l'environnement, 11 août 2015, reconnaissant la responsabilité de l'investisseur sans se prononcer sur le quantum). Sur le régime de responsabilité applicable au cas de l'investisseur violant le droit international des droits de l'homme, voir supra, I, C (Principes généraux).

382. Sur ce point, voir Makane Moïse MBENGUE, "Les obligations des investisseurs étrangers ", in SFDI, L'entreprise multinationale et le droit international, Paris, Pedone, 2017, pp. 295-337, spé. p. 321, jugeant que l'approche ici suivie constitue une "porte d'entrée étroite pour les obligations des investisseurs " et appelant à l'ouverture d'" une fenêtre juridique " plus large par la formulation d'obligations spécifiques des investisseurs au sein même des traités d'investissement.

383. Conseil des droits de l'homme, Principes directeurs relatifs aux entreprises et aux droits de l'homme, doc. A/HRC/17/31, 2011, principes 1 (État) et 11 (entreprise).

384. CIRDI, BSG Resources Ltd, BSG Resources (Guinea) Ltd et BSG Resources (Guinea) SARL c. Guinée, $\mathrm{n}^{\circ} \mathrm{ARB} / 14 / 22$, décision sur la demande de récusation de tous les membres du tribunal, 28 décembre 2016. 
l'État met en cause les liens conservés avec son ancien cabinet ${ }^{385}$. Dans la mesure où elles se sont toutes soldées par un rejet, alors qu'au fil des années l'ensemble des liens entre cet arbitre et son ancien cabinet sont passés au crible, ces demandes pourraient apparaître abusives. D'autres, peut-être le tiers raisonnable auxquels se réfêrent les arbitres, pourraient toutefois se demander si, à s'en tenir aux apparences, il ne se trouvait pas d'arbitre disponible sans lien avec un cabinet d'avocats impliqué dans plusieurs procédures dirigées contre le Venezuela ${ }^{386}$...

Sur le plan procédural, l'affaire $E D F$ et Saur a été l'occasion de revenir sur la façon dont les comités $a d$ hoc abordent ces questions d'indépendance et d'impartialité des arbitres dans le cadre de demandes d'annulation. L'Argentine cherchait à remettre en cause deux des arbitres qui s'étaient prononcés au fond. La première avait déjà fait l'objet d'une demande de récusation, rejetée par la majorité du tribunal, au contraire du second, dont l'Argentine considérait que des faits apparus après la sentence remettaient en cause l'indépendance. Le comité devait donc déterminer quel devait être son rôle en la matière à ce stade tardif de la procédure. Au contraire du comité de l'affaire Azurix ${ }^{387}$, il considéra qu'il devait non seulement contrôler la compatibilité de la procédure de récusation déjà menée avec les exigences de l'article 52, mais que l'examen de l'indépendance et de l'impartialité des arbitres relevait doublement de son rôle de gardien de l'intégrité de l'arbitrage. Dépendance et/ou partialité révéleraient en effet un « vice dans la constitution du tribunal » ou seraient constitutives d'une «violation grave d'une règle fondamentale de procédure ", justifiant ainsi l'annulation de la sentence rendue ${ }^{388}$. Malgré ce constat général, le comité distingue selon qu'il s'agit d'une demande nouvelle ou ayant déjà fait l'objet d'une décision. Dans le premier cas, le comité est placé dans la situation de la majorité du tribunal, saisi d'une demande de récusation : sous réserve que la demande ait été introduite promptement une fois les faits connus, il doit examiner la situation avec les yeux d'un tiers raisonnable ${ }^{389}$. Dans le second cas en revanche, le comité doit certes préserver l'intégrité de la procédure mais sans se muer en juge d'appel : il pense pouvoir le faire en réalisant un contrôle que l'on pourrait qualifier de restreint sur la décision de récusation initiale puisqu'il se contente d'en examiner le caractère raisonnable ${ }^{390}$. La raison est alors grandement sollicitée afin d'apprécier si la majorité d'un tribunal arbitral s'est montrée raisonnable en jugeant qu'un tiers raisonnable ne douterait pas de l'indépendance ou de l'impartialité d'un arbitre.

385. CIRDI, ConocoPhillips Petrozuata BV et al. c. Venezuela, ${ }^{\circ}$ ARB/07/30, décision sur la demande de récusation de L. Yves Fortier, QC, 15 mars 2016 ; décision sur la demande de récusation de L. Yves Fortier, 26 juillet 2016 ; CIRDI, Fábrica de Vidrios La Andes, CA et Owens-Illinois de Venezuela, CA c. Venezuela, $\mathrm{n}^{\circ} \mathrm{ARB} / 12 / 21$, décision sur la demande de récusation de L. Yves Fortier, 28 mars 2016 ; décision sur la demande de récusation de L. Yves Fortier, 12 septembre 2016.

386. Cf. CIRDI, Blue Bank c. Venezuela, ${ }^{\circ} \mathrm{ARB} / 12 / 20$, décision sur la demande de récusation de la majorité du tribunal, 12 novembre $2013, \S 69$, jugeant que, confronté à l'appartenance d'un arbitre à la filiale espagnole d'un cabinet américain impliqué dans un différend parallèle contre le même Etat défendeur, " a third party would find an evident or obvious appearance of lack of impartiality".

387. Comité ad hoc CIRDI, Azurix c. Argentine, $\mathrm{n}^{\circ} \mathrm{ARB} / 01 / 12$, décision sur l'annulation, $1^{\mathrm{er}}$ septembre $2009, \S \S 280-281$, jugeant que toute autre demande postérieure à la sentence mettant en cause l'indépendance ou l'impartialité des arbitres devait faire l'objet d'une procédure de révision.

388. Comité ad hoc CIRDI, EDF SA et SAUR International SA c. Argentine, n ${ }^{\circ}$ ARB/03/23, décision sur l'annulation, 5 février 2016, §§ 117-128.

389. Ibid., $\$$ 130-137 et 165-175 pour l'application, considérant que le fait que l'arbitre concerné travaille dans un cabinet d'avocats devenu conseil d'une société engagée dans un différend contre l'Argentine entre la date de finalisation de la sentence et celle de sa publication n'a pas fait naître de conflit d'intérêts susceptible d'avoir un effet sur cette sentence.

390. Ibid., $\S \S 137-146$ et $\S \S 147-164$ pour l'application, considérant comme raisonnable la décision initiale selon laquelle le fait que l'arbitre concernée soit membre du conseil d'administration d'une banque ayant une participation mineure $(1,5 \%)$ dans la société-mère de la demanderesse ne serait pas de nature à remettre en cause son indépendance et son impartialité aux yeux d'un tiers raisonnable. 


\section{Procédures d'urgence}

Les procédures d'urgence sont régulièrement utilisées dans le contentieux arbitral transnational. On sait que les tribunaux ${ }^{391}$ saisis d'une demande principale ont le pouvoir de prononcer des mesures provisoires considérées comme obligatoires malgré l'incertitude des textes ${ }^{392}$ lorsque certaines conditions sont réunies. Cellesci tiennent classiquement, d'une part, à l'existence d'un droit que la sentence est susceptible de reconnaître (ce qui implique un examen prima facie par le tribunal) et, d'autre part, à la nécessité de la mesure pour sauvegarder ledit droit (qui se décline en appréciation de la menace, de l'urgence et de la proportionnalité). Ceci est bien connu. Un aspect remarquable se dégage toutefois de la pratique de l'année : la plupart des mesures sollicitées portent non sur les droits substantiels qui sont en litige ${ }^{393}$ mais sur les droits procéduraux qui sont liés au litige.

Ainsi les parties sollicitent-elles des arbitres qu'ils ordonnent à leurs adversaires un cautionnement des frais d'arbitrage ${ }^{394}$, le paiement des avances nécessaires pour couvrir lesdits frais ${ }^{395}$ ou leur interdisent de divulguer des informations relatives à la procédure ${ }^{396}$. Les investisseurs tendent également à demander aux tribunaux qu'ils exigent des États mis en cause la suspension de procédures pénales engagées en lien avec la procédure arbitrale. Sur ce point, les arbitres sont à la recherche d'un équilibre entre le souci de ne pas interférer trop fortement «with the exercise of a sovereign State's rights and duties to investigate and prosecute crime » et la crainte que ce pouvoir de punir ne soit détourné pour remettre en cause la procédure arbitrale ${ }^{397}$. Ils pensent le trouver en appréciant strictement la nécessité de la mesure provisoire sollicitée et en affirmant exercer une vigilance continue sur le déroulement de la procédure. Ainsi, dans l'affaire Hydro, le tribunal a d'abord considéré qu'il était absolument nécessaire d'ordonner la suspension de la procédure pénale engagée contre les requérants en Albanie ainsi que celle de la procédure d'extradition les visant au Royaume-Uni avant de juger que cette ordonnance pouvait être amendée, compte tenu du fait que les autorités britanniques avaient rejeté la demande d'extradition albanaise ${ }^{398}$. De manière comparable, les arbitres de l'affaire Teinver ont refusé d'ordonner les mesures conservatoires

391. Se posant la question de savoir si les comités ad hoc peuvent le faire, sans y répondre car les conditions ne sont en tout état de cause pas réunies, Comité ad hoc CIRDI, Bernhard von Pezold et al. $c$. Zimbabwe, $\mathrm{n}^{\circ} \mathrm{ARB} / 10 / 15$, décision sur la demande de mesures provisoires du demandeur, 17 mars 2016 , $\S \S 30-32$; illustrant la possibilité qu'un arbitre d'urgence se prononce avant la constitution du tribunal arbitral ouverte par certains règlements d'arbitrage, Ch. comm. Stockholm, Evrobalt LLC c. Moldavie, arbitrage d'urgence EA 2016/082, sentence sur les mesures d'urgence, 30 mai 2016 ; Ch. comm. Stockholm, Kompozit LLC c. Moldavie, EA 2016/095, sentence d'urgence sur les mesures provisoires, 14 juin 2016.

392. Pour un rappel de la discussion et de cette solution, CIRDI, Transglobal Green Energy, LLC et Transglobal Green Energy de Panama, SA c. Panama, ${ }^{\circ}$ ARB/13/28, décision sur la demande de mesures conservatoires du défendeur relative au cautionnement pour frais, 25 janvier 2016, § 25 ; CIRDI, Valle Verde Sociedad Financiera SL c. Venezuela, ${ }^{\circ} \mathrm{ARB} / 12 / 21$, décision sur les mesures provisoires, 25 janvier 2016, §§ 72-75 ; CIRDI, United Utilities (Talinn) BV et Aktsiaselts Tallinna Vesi c. Estonie, ARB/14/24, décision sur la demande de mesures provisoires, 12 mai 2016, § 109.

393. Voir toutefois les affaires Evrobalt LLC et Kompozit LLC, précit.

394. CIRDI, Transglobal Green Energy, LLC et Transglobal Green Energy de Panama, SA c. Panama, $\mathrm{n}^{\circ} \mathrm{ARB} / 13 / 28$, décision sur la demande de mesures conservatoires du défendeur relative au cautionnement pour frais, 25 janvier 2016, rejet.

395. CIRDI, Valle Verde Sociedad Financiera SL c. Venezuela, ${ }^{\circ}$ ARB/12/21, décision sur les mesures provisoires, 25 janvier 2016, rejet, le règlement prévoyant qu'en cas de défaut de paiement de l'avance, il revient à l'autre partie de la couvrir puis au tribunal de statuer in fine sur la répartition des frais.

396. CIRDI, United Utilities (Talinn) BV et Aktsiaselts Tallinna Vesi c. Estonie, ARB/14/24, décision sur la demande de mesures provisoires, 12 mai 2016, admission partielle.

397. CIRDI, Hydro Srl et al. c. Albanie, n ARB/15/28, ordonnance sur les mesures conservatoires, 3 mars $2016, \S 3.14$.

398. CIRDI, Hydro Srl et al. c. Albanie, $\mathrm{n}^{\circ} \mathrm{ARB} / 15 / 28$, ordonnance sur les mesures conservatoires, 3 mars 2016 et décision intermédiaire, $1^{\mathrm{er}}$ septembre 2016. 
sollicitées tout en affirmant qu'ils pourraient réexaminer la question à la demande des investisseurs en fonction des développements de la procédure pénale ${ }^{399}$.

\section{Caractère définitif des sentences}

Célérité et efficacité font partie des vertus habituellement prêtées à l'arbitrage pour le règlement des différends opposant investisseurs et États. La procédure se clôt ainsi par une sentence définitive, qui statue sur les prétentions des parties et répartit les coûts de l'arbitrage lui-même et ceux de la représentation des parties. Sur ces questions de coût, les décisions rendues dans l'année confirment que les tribunaux tendent à user de leur marge d'appréciation pour tenir compte de l'issue finale de la procédure, pas toujours univoque, et du comportement des parties en cours d'instance ${ }^{400}$, tandis que l'affaire Transglobal Green met en lumière les difficultés auxquelles peuvent être confrontés les États qui cherchent à recouvrer les sommes dues à ce titre ${ }^{401}$, justifiant que le conseil administratif du CIRDI ait inscrit, à l'initiative du Panama, cette question parmi celles sur lesquelles pourraient porter les amendements au Règlement CIRDI ${ }^{402}$.

Bien que les procédures tendent à s'allonger, le souci de célérité et d'efficacité justifie encore largement que les sentences rendues ne puissent faire l'objet d'un appel mais tout au plus, dans le cadre du CIRDI, d'une procédure d'annulation destinée à préserver l'intégrité de la procédure arbitrale. Le recours à cette procédure se fait toutefois régulier, ainsi que l'indique la publication de neuf décisions rendues dans l'année, dont sept ont abouti à un rejet ${ }^{403}$ quand deux ont été accueillies ${ }^{404}$. Ces décisions sont autant d'occasions de rappeler que l'annulation ne constitue pas un appel. D'une part, parce que le comité ne procède pas au réexamen des questions de droit et de fait tranchées par le tribunal mais se prononce sur la base de motifs d'annulation étroitement définis bien qu'ils puissent se chevaucher ${ }^{405}$. À cet égard, les décisions d'annulation de l'année tendent à indiquer l'inconfort des arbitres au stade de l'évaluation des préjudices, l'une mettant en évidence un défaut de motifs

399. CIRDI, Teinver SA, Transportes de Cercanías SA et Autobuses Urbanos del Sur SA c. Argentine, ARB/09/1, décision sur les mesures conservatoires, 8 avril 2016.

400. Pour un exemple, CIRDI, The Renco Group, Inc. c. Pérou, $\mathrm{n}^{\circ} \mathrm{UNCT} / 13 / 1$, sentence finale, 9 novembre 2016.

401. Alors que le demandeur était une société ne disposant plus d'actifs, le tribunal a refusé d'ordonner à titre conservatoire un cautionnement pour les frais d'arbitrage puis a rejeté la demande pour abus de procédure, condamnant alors le demandeur à assumer l'ensemble des frais d'arbitrage (CIRDI, Transglobal Green Energy, LLC et Transglobal Green Energy de Panama, SA c. Panama, n ARB/13/28, décision sur la demande de mesures conservatoires du défendeur relative au cautionnement pour frais, 25 janvier 2016 ; sentence, 2 juin 2016). FR.pdf.

402. https://icsid.worldbank.org/fr/Documents/The\%20ICSID\%20Rules\%20Amendment\%20Process.

403. Comité ad hoc CIRDI, Adem Dogan c. Turkménistan, n ARB/09/9, décision sur l'annulation, 15 janvier 2016 ; Comité ad hoc CIRDI, Total SA c. Argentine, n ARB/04/01, décision sur l'annulation, $1^{\text {er }}$ février 2016 ; Comité ad hoc CIRDI, EDF SA et SAUR International SA c. Argentine, n ARB/03/23, décision sur l'annulation, 5 février 2016 ; Comité ad hoc CIRDI, Ion Micula et al. c. Roumanie, n ARB/05/20, décision sur l'annulation, 26 février 2016 ; Comité ad hoc CIRDI, Antoine Abou Lahoud et al. c. RDC, $\mathrm{n}^{\circ} \mathrm{ARB} / 10 / 4$, décision sur l'annulation, 29 mars 2016 ; Comité ad hoc CIRDI, Postová banka, a.s. et Istrokapital SE c. République Hellénique, $\mathrm{n}^{\circ} \mathrm{ARB} / 13 / 8$, décision sur l'annulation, 29 septembre 2016 ; Comité ad hoc CIRDI, SAUR International SA c. Argentine, $\mathrm{n}^{\circ} \mathrm{ARB} / 04 / 4$, décision sur l'annulation, 19 décembre 2016.

404. Comité ad hoc CIRDI, TECO Guatemala Holdings LLC c. Guatemala, n ARB/10/23, décision sur l'annulation, 5 avril 2016 ; Comité ad hoc CIRDI, Tidewater Inc., Tidewater Investment SRL, Tidewater Caribe, $C A$, et al. c. Venezuela, $\mathrm{n}^{\circ} \mathrm{ARB} / 10 / 5$, décision sur l'annulation, 27 décembre 2016.

405. Comp. Comité ad hoc CIRDI, Total SA c. Argentine, $\mathrm{n}^{\circ} \mathrm{ARB} / 04 / 01$, décision sur l'annulation, $1^{\mathrm{er}}$ février 2016 et Comité ad hoc CIRDI, EDF SA et SAUR International SA c. Argentine, n ${ }^{\circ}$ ARB/03/23, décision sur l'annulation, 5 février 2016, le premier abordant successivement les motifs d'annulation de l'article 52, le second les griefs matériels dirigés contre la sentence. 
s'agissant du refus d'évaluer la perte subie par le demandeur ${ }^{406}$, l'autre révélant une erreur dans le calcul du préjudice, erreur admise par le tribunal à l'occasion d'une procédure de révision sans pour autant en tirer de conséquences ${ }^{407}$. D'autre part, parce que la procédure ne permet en principe pas la réformation des sentences mais uniquement leur annulation. Sur ce point, trois éléments peuvent être mis en lumière cette année : les comités disposent d'une marge d'appréciation quant aux conséquences à tirer d'un vice de la sentence ${ }^{408}$; ils peuvent procéder à une annulation partielle qui confine parfois à la réformation ${ }^{409}$; une telle annulation partielle peut conduire à la saisine d'un nouveau tribunal, alors confronté à la difficile tâche de faire le départ entre les parties annulées et celles qui ne l'ont pas été, seulement sujettes à interprétation ${ }^{410}$. Bien qu'il ne s'agisse sans doute pas du lot commun, l'issue de l'affaire Victor Pey Casado conduit d'ailleurs à relativiser l'efficience de l'arbitrage transnational : dix-neuf ans après l'introduction de la demande, le tribunal conclut que le fait illicite constaté dans la sentence initiale partiellement annulée ne peut donner lieu à aucune indemnisation, l'investisseur n'ayant pas fait la preuve du préjudice causé par ce fait particulier ! Entre temps, une première sentence et une décision d'annulation partielle auront été rendues, mais aussi des décisions sur les demandes de mesures provisoires, de disqualification et de révision, moyennant quelques millions de dollars ${ }^{411}$.

Par-delà le mécanisme d'annulation, on peut être frappé par la multiplication des demandes tendant à revenir, d'une manière ou d'une autre, sur tout ou partie des décisions rendues au cours de la procédure. D'abord, les parties peuvent solliciter les arbitres avant le prononcé de la sentence finale afin qu'ils reviennent sur certains constats opérés dans des décisions intermédiaires à la lumière de faits nouveaux. La majorité du tribunal de l'affaire ConocoPhillips a confirmé qu'elle estimait ne pas en avoir le pouvoir, alors que le Venezuela lui demandait de prendre en compte des révélations ultérieures pour revenir sur le constat d'expropriation illicite fait en $2013^{412}$. À l'inverse, le tribunal de l'affaire Standard Chartered a accepté de réexaminer sa décision initiale, à la lumière des faits nouveaux qui n'avaient pas été portés à sa connaissance par la défenderesse, avant de se prononcer sur le quantum ${ }^{413}$. Les décisions préalables à la sentence ne sont pas formellement revêtues de l'autorité de la chose jugée, même si elles ont vocation à intégrer la sentence. Dès lors, entre efficacité procédurale et exactitude matérielle, la balance ne semble pas devoir être fixée a priori, de sorte que l'approche in casu du tribunal de l'affaire Standard Chartered convainc davantage ${ }^{414}$. C'est d'autant plus le cas

406. Comité ad hoc CIRDI, TECO Guatemala Holdings LLC c. Guatemala, n ARB/10/23, décision sur l'annulation, 5 avril 2016.

407. Comité ad hoc CIRDI, Tidewater Inc., Tidewater Investment SRL, Tidewater Caribe, CA, et al. c. Venezuela, $\mathrm{n}^{\circ} \mathrm{ARB} / 10 / 5$, décision sur l'annulation, 27 décembre 2016.

408. En ce sens, Comité ad hoc CIRDI, EDF SA et SAUR International SA c. Argentine, $\mathrm{n}^{\circ} \mathrm{ARB} / 03 / 23$, décision sur l'annulation, 5 février 2016, § 73.

409. Comité ad hoc CIRDI, Tidewater Inc., Tidewater Investment SRL, Tidewater Caribe, CA, et al. c. Venezuela, $\mathrm{n}^{\circ} \mathrm{ARB} / 10 / 5$, décision sur l'annulation, 27 décembre 2016, $\$ \S 202-223$.

410. CIRDI, Victor Pey Casado et President Allende Foundation c. Chili, $\mathrm{n}^{\circ} \mathrm{ARB} / 98 / 2$, sentence, 13 septembre 2016.

411. Ibid.

412. CIRDI, ConocoPhillips Petrozuata BV et al. c. Venezuela, $\mathrm{n}^{\circ} \mathrm{ARB} / 07 / 30$, décision sur la demande de réexamen de la décision du tribunal du 10 mars 2014, 9 février 2016, §§ 20-38, et op. diss. Bucher. La décision contestée a été rendue en 2013 mais la majorité a déjà refusé de revenir sur les constats opérés en 2014. Voir cette chronique, cet Annuaire, 2014, pp. 613-615.

413. CIRDI, Standard Chartered Bank (Hong Kong) Ltd c. Tanzania Electric Supply Company Ltd, $\mathrm{n}^{\circ} \mathrm{ARB} / 10 / 20$, sentence, 12 septembre 2016.

414. Voir aussi CIRDI, Getma International, NCT Necotrans, Getma International Investments, NCT Infrastructure \& Logistique c. Guinée, $\mathrm{n}^{\circ} \mathrm{ARB} / 11 / 29$, sentence, 16 août 2016, § 180 ; CIRDI, Pac Rim Cayman LLC c. République du Salvador, $\mathrm{n}^{\circ}$ ARB/09/12, sentence, 14 octobre 2016, $\$ 5.36-5.37$; CIRDI, Hochtief AG c. Argentine, ${ }^{\circ}$ ARB/07/31, sentence, 19 décembre 2016, §§ 49-69. 
que, dans le mécanisme CIRDI, les parties peuvent encore recourir aux procédures prévues aux articles 49 et 51 de la convention de Washington pour rechercher une décision supplémentaire, une rectification ou une révision de la sentence, ce dont ils ne se privent pas ${ }^{415}$ sans que l'on puisse les en blâmer ${ }^{416}$.

415. CIRDI, İçkale İnşaat Limited Şirketi c. Turkménistan, ${ }^{\circ}$ ARB/10/24, décision sur la demande de décision supplémentaire et de rectification de la sentence, 4 octobre 2016.

416. Comité ad hoc CIRDI, Tidewater Inc., Tidewater Investment SRL, Tidewater Caribe, CA, et al. c. Venezuela, $\mathrm{n}^{\circ} \mathrm{ARB} / 10 / 5$, décision sur l'annulation, 27 décembre 2016, § 119. 